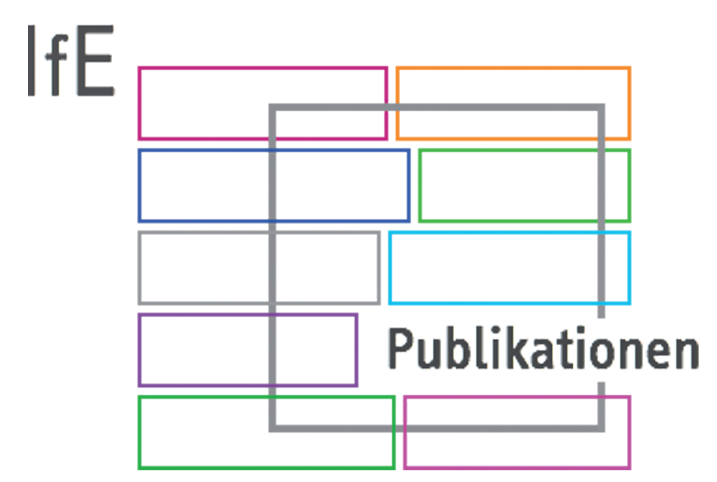

Göttinger Beiträge zur erziehungswissenschaftlichen

Forschung

40

\title{
Skalendokumentation des Projekts EFBI
}

\author{
Einstellungen von FSJler*innen \\ zu Behinderung und Inklusion
}

\author{
Ariane S. Willems \\ Karina Meyer \\ Moritz Frechen
}



Veröffentlicht als

Göttinger Beiträge zur erziehungswissenschaftlichen Forschung, 40

Institut für Erziehungswissenschaft 2021

\author{
Ariane S. Willems, Karina Meyer \& \\ Moritz Frechen
}

Skalendokumentation des Projekts EFBI

Einstellungen von FSJler*innen

zu Behinderung und Inklusion

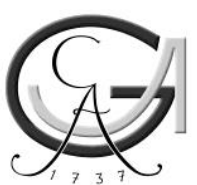

Georg-August-Universität Göttingen 2021

Dieses Werk ist lizenziert unter einer

Creative Commons Namensnennung - Weitergabe unter gleichen Bedingungen 4.0 International Lizenz.

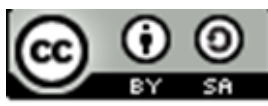


Bibliografische Information der Deutschen Nationalbibliothek

Die Deutsche Nationalbibliothek verzeichnet diese Publikation in der Deutschen Nationalbibliografie; detaillierte bibliografische Daten sind im Internet über <http://dnb.dnb.de $>$ abrufbar.

Anschrift der Autor*innen

Georg-August-Universität Göttingen

Institut für Erziehungswissenschaft

Lehrstuhl für Empirische Bildungsforschung

mit dem Schwerpunkt Schul- und Unterrichtsforschung

Waldweg 26

37073 Göttingen

Satz und Layout: Karina Meyer

ISSN: $2198-2384$

doi: $10.17875 /$ gup2021-1761 


\section{Inhaltsverzeichnis}

A Überblick zum Projekt EFBI 6

A.1 Hintergrund und Ziele des Projektes......................................................... 6

A.2 Projektdesign und Stichprobe.................................................................... 9

A.3 Eingesetzte Fragebögen............................................................................. 11

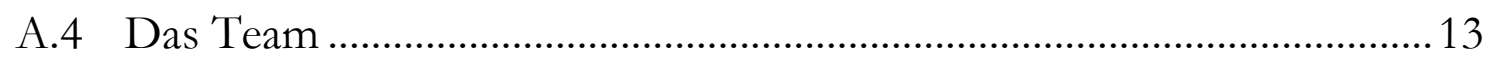

A.5 Publikationen, Vorträge und Qualifikationsarbeiten .................................14

B Hinweise zur Skalendokumentation EFBI $\quad 18$

B.1 Aufbau der Skalendokumentation..........................................................18

B.2 Methodische Grundlagen der Item- und Skalenanalysen........................20

1 Einstellungen zu Behinderung 23

1.1 Einstellungen zu (Menschen mit) Behinderung .......................................23

1.1.1 Menschen mit Behinderung als Bereicherung............................................................. 23

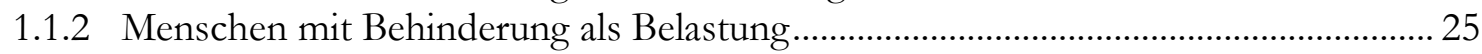

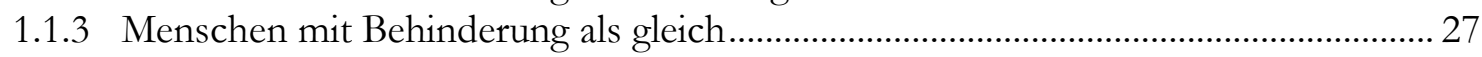

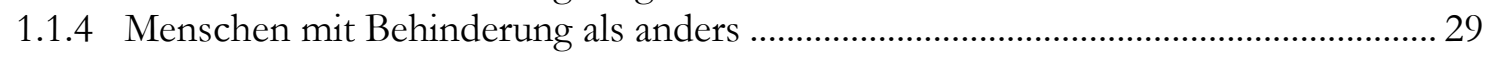

1.1.5 Unsicherheit im Kontakt mit Menschen mit Behinderung........................................ 31

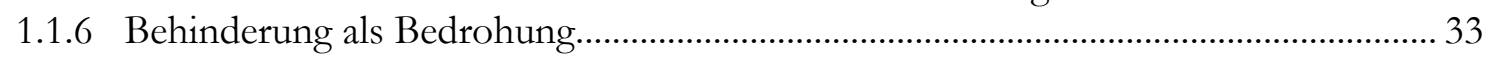

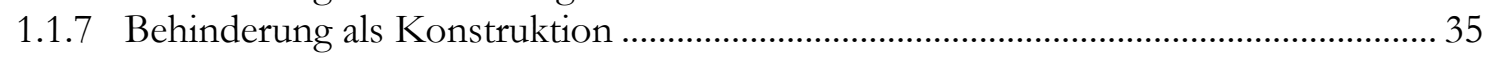

1.1.8 Strukturmodell Einstellungen zu (Menschen mit) Behinderung .................................. 37

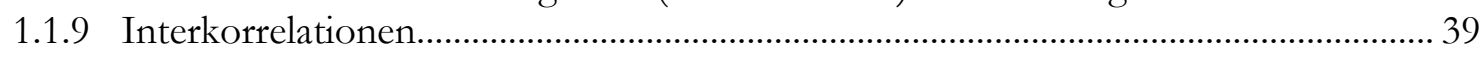

1.2 Ingroup/Outgroup: Ähnlichkeit von Menschen mit und ohne Behinderung............................................................................................... 41

1.3 Soziale Distanz zu Menschen mit Behinderung....................................... 43

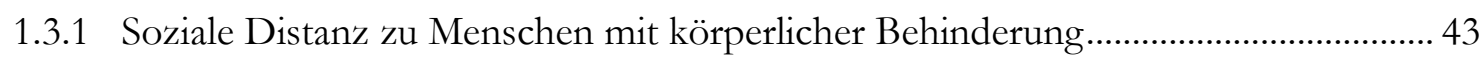

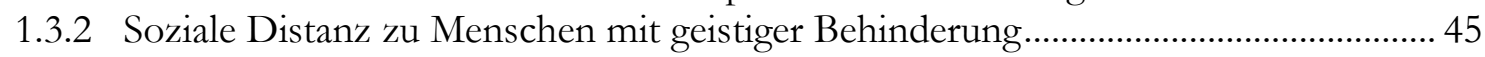

1.3.3 Soziale Distanz zu Menschen mit psychischer Erkrankung ........................................ 47

1.4 Multidimensional Attitudes Scale (MAS) ................................................... 49

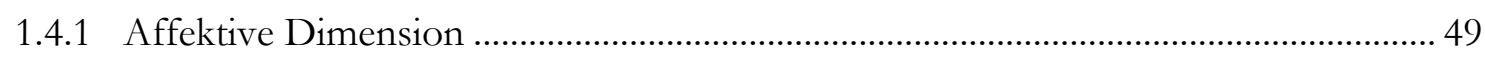

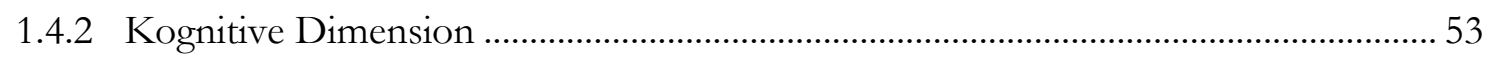

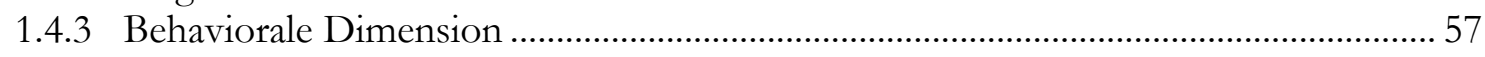

2 Einstellungen zu Inklusion $\quad 61$

2.1 Befürwortung von Inklusion im Sinne der UN-BRK ..............................61

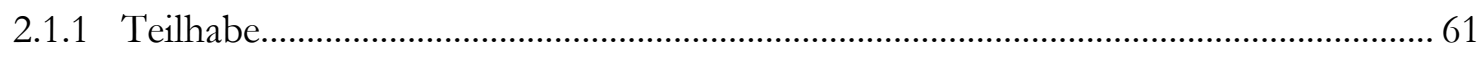

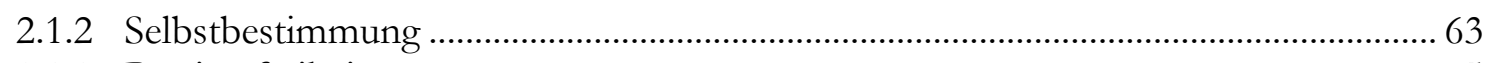

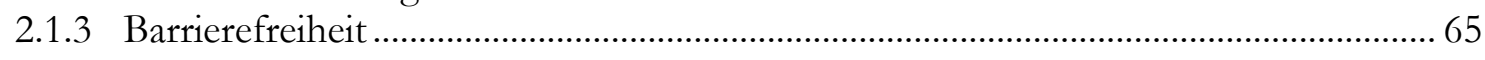

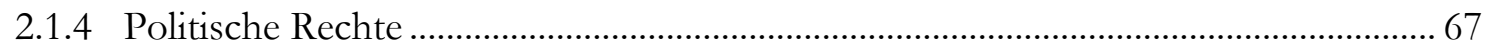


2.1.5 Strukturmodell Befürwortung von Inklusion ................................................................69

2.2 Befürwortung von schulischer Inklusion ............................................71

2.2.1 Schulische Inklusion von Schüler*innen mit körperlicher Behinderung.....................71

2.2.2 Schulische Inklusion von Schüler*innen mit geistiger Behinderung..............................77

2.2.3 Schulische Inklusion von Schüler*innen mit psychischer Erkrankung.........................83

2.3 Befürwortung von Inklusion bei spezifischer Behinderung ................... 88

3 Arbeitsbezogene Selbstwirksamkeitserwartung 89

4 Wissen zu Inklusion $\quad 93$

4.1 Inklusive Gesellschaft ..................................................................... 93

4.2 UN-Behindertenrechtskonvention..................................................... 94

4.3 Exklusion/Separation/Integration/Inklusion ..................................... 95

5 Angaben zur Stichprobe $\quad 97$

5.1 Personenbezogene Angaben ............................................................ 97

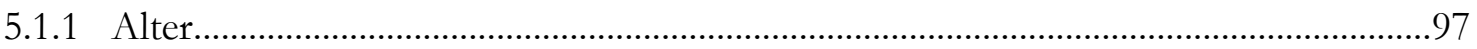

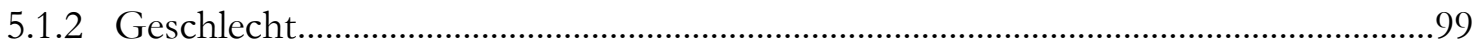

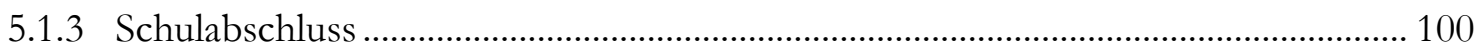

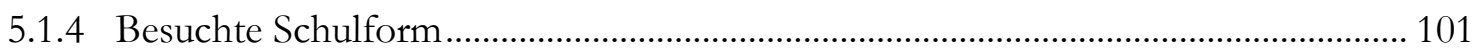

5.2 Rahmendaten zum Freiwilligendienst ............................................... 103

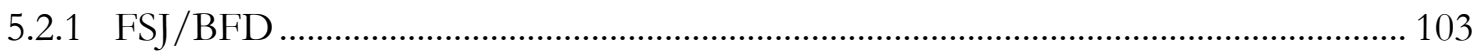

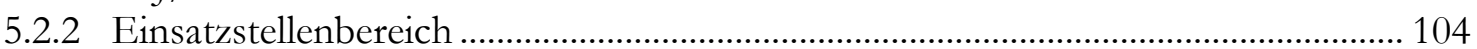

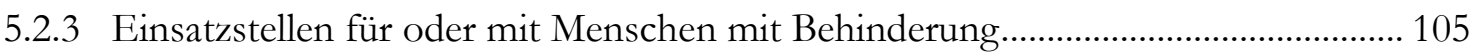

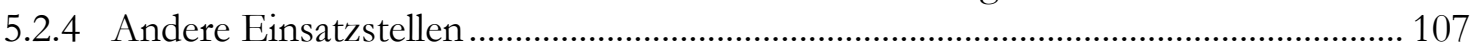

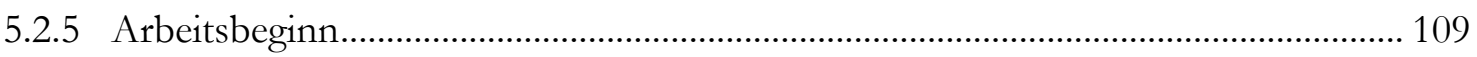

5.3 Individuelle Eingangsvoraussetzungen und Kontextvariablen im

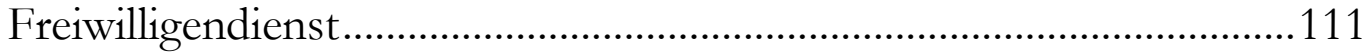

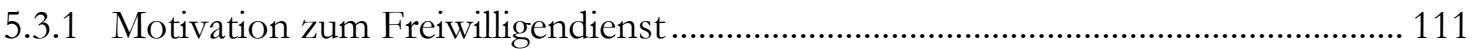

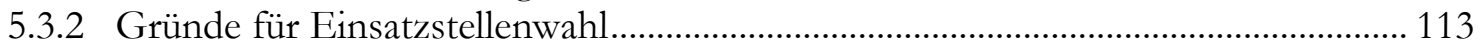

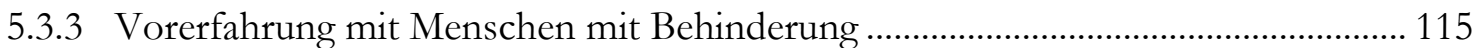

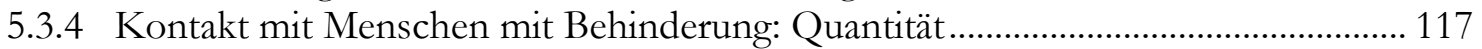

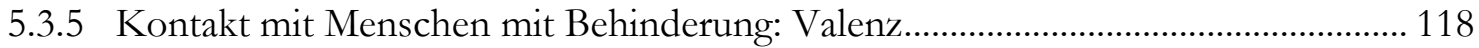

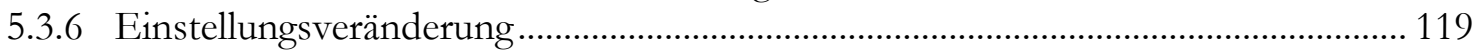

$\begin{array}{ll}\text { C Literaturverzeichnis } & 120\end{array}$ 


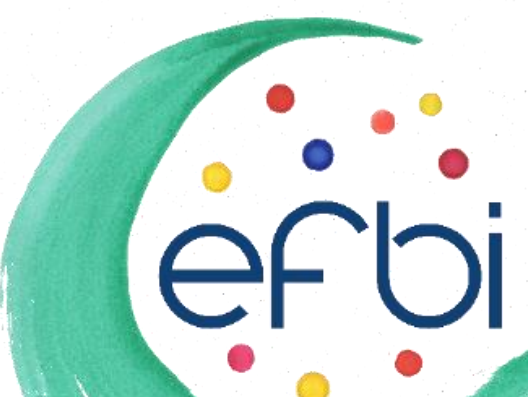

Projektbeschreibung 


\section{A Überblick zum Projekt EFBI}

\section{A.1 Hintergrund und Ziele des Projektes}

Die Studie EFBI (Einstellungen von FSJler*innen zu Behinderung und Inklusion) stellt eine längsschnittliche Studie im Bereich außerschulischer Bildungsforschung dar, die behinderungs- und inklusionsbezogene Einstellungen von Jugendlichen und jungen Erwachsenen im Verlauf eines Freiwilligendienstes untersucht. ${ }^{1}$

Positive Einstellungen zu Behinderung und Inklusion gelten als zentrale Gelingensfaktoren für die erfolgreiche Implementierung von Inklusion im Sinne der UNBehindertenrechtskonvention (z. B. Forlin, Earle, Loreman, \& Sharma, 2001; Seifried \& Heyl, 2016). Diese Annahme, die auch in der Konvention selbst Ausdruck findet ( 8 8: Bewusstseinsbildung), kann sich auf zahlreiche sozialwissenschaftliche und sozialpsychologische Studien zum Zusammenhang von Einstellungen und Verhalten stützen (z. B. Eagly \& Chaiken, 1998; Fazio, 1990; Fishbein \& Ajzen, 2010; für einen Überblick siehe Glaser \& Bohnet, 2016). Entsprechend sind Einstellungen von Akteur*innen im Bildungsbereich infolge der Ratifizierung der UNBehindertenrechtskonvention verstärkt in den Blick der empirischen Bildungsforschung gerückt. Zu schulischen Akteur*innen (insbesondere Lehrkräften und Lehramtsstudierenden) liegt bereits eine Vielzahl von Befunden zu Einstellungen und hiermit assoziierten personen- und kontextbezogenen Merkmalen vor, wobei die Erhebungen zumeist querschnittlich angelegt sind und nur bedingt Aussagen über kausale Zusammenhänge ermöglichen (z. B. Bosse \& Spörer 2014; Hellmich, Görel \& Schwab 2016; McElvany, Schwabe, Hartwig \& Igler, 2018; für Überblicke über

\footnotetext{
${ }^{1}$ Das Projekt wird aus Eigenmitteln des Lehrstuhls Empirische Bildungsforschung mit dem Schwerpunkt Schul- und Unterrichtsforschung an der Georg-August-Universität (Institut für Erziehungswissenschaft) finanziert.
} 
deutschsprachige bzw. internationale Publikationen siehe Ruberg \& Porsch, 2017 bzw. Pit-ten Cate, Markova, Krischler \& Krolak-Schwerdt, 2018).

An systematischen Längsschnittstudien zu Einstellungsentwicklungen im Kontext Inklusion mangelt es bislang. Theoretisch begründete Annahmen über diesbezügliche Einflussfaktoren lassen sich aus verschiedenen sozialpsychologischen Einstellungstheorien ableiten. Gemäß dem zentralen Postulat der Kontakthypothese (Allport, 1954), das empirisch vielfach belegt werden konnte (für einen Überblick siehe z. B. Aydin, Pfundmair, Agthe, Lermer \& Frey, 2016), werden negative Vorurteile durch Intergruppenkontakt generell abgebaut, wobei die Intensität und die Art des Kontakts sowie seine subjektive Bewertung eine zentrale Rolle spielen (z. B. Barr \& Bracchitta, 2012; McManus, Feyes \& Saucier, 2010; siehe auch Pettigrew, 1998). Gleichzeitig kann aber auch allein die zunehmende Vertrautheit mit zuvor fremden Personen positivere Einstellungen gegenüber diesen bewirken (mere exposure effect, Zajonc, 1968). Auch sind z. B. über Persuasion bewusst induzierte Einstellungsveränderungen möglich; ihr Erfolg hängt insbesondere damit zusammen, wie die entsprechende Informationsquelle subjektiv bewertet wird (McGuire, 1969; Werth \& Mayer, 2008). Generell treten Einstellungsveränderungen mit höherer Wahrscheinlichkeit auf, wenn die entsprechenden Einstellungen nur schwach ausgeprägt sind (Petty \& Cacioppo, 1986) oder wenn eine Dissonanz zwischen Einstellungen und Handeln erlebt wird (Festinger, 1957). Bewusst vorgenommene Einstellungsveränderungen können erfolgen, wenn es sich um explizite Einstellungen handelt (d. h. der Person selbst zugängliche Einstellungen) und sowohl die Motivation als auch die Fähigkeit zur Änderung bestehen (Maio \& Haddock, 2015).

Vor dem Hintergrund theoretischer Modelle aus der sozialpsychologischen Einstellungsforschung und empirischer Befunde aus der Inklusionsforschung widmet sich die Studie EFBI einer Zielgruppe, die zwar Gegenstand mehrerer staatlich in Auftrag gegebener Evaluationen ist (Engels, Leucht \& Machalowski, 2008; Huth, Aram, Wagner, Engels \& Maur, 2015; Rahrbach, Wüstendörfer \& Arnold, 1998), bislang aber nicht im Fokus empirischer Bildungsforschung stand: Jugendliche und junge Erwachsene, die ein Freiwilliges Soziales Jahr (FSJ) absolvieren. ${ }^{2}$ Das FSJ als staatlich gefördertes, strukturiertes Freiwilligenprogramm für junge Menschen bis 27 Jahren hat zum Ziel, die „Bildungs- und Beschäftigungsfähigkeit der Freiwilligen“ zu fördern ( $55 \mathrm{Ju}$ gendfreiwilligendienstegesetz (JFDG)) sowie u. a. „soziale, kulturelle und interkulturelle Kompetenzen zu vermitteln“ (\$3) und ist in diesem Sinne im Bereich außerschulischer Bildung zu verorten. Dies findet Ausdruck darin, dass die Freiwilligen während des Jahres pädagogisch begleitet werden (\$3) und neben ihrer praktischen Hilfstätigkeit in sozialen Einrichtungen (FSJ-Einsatzstelle) insgesamt mindestens 25 Seminartage absolvieren (\$5). In den letzten Jahrzehnten ist die Teilnehmendenzahl am FSJ stark angestiegen auf etwa 56000 im Jahr 2017 (Autorengruppe Bildungsberichtserstattung, 2018). Das FSJ stellt damit das teilnehmerstärkste staatlich geförderte Freiwilligenprogramm dar; insgesamt erreichen diese Programme (neben dem FSJ z. B. das Freiwillige

\footnotetext{
2 Durch die Zusammensetzung der Seminargruppen wurden auch Absolvierende des Bundesfreiwilligendienstes (BFD) befragt, diese machen allerdings nur einen sehr geringen Anteil an der Gesamtstichprobe aus (siehe Kapitel 5.2.1).
} 
Ökologische Jahr, das Freiwillige Kulturelle Jahr sowie Auslandsfreiwilligenprogramme) unter Jugendlichen und jungen Erwachsenen etwa 11\% eines Altersjahrgangs (ebd.), womit ihnen als nonformalem Bildungsangebot insbesondere für die Phase zwischen Schule und Ausbildung eine hohe Bedeutung zukommt.

Zur Untersuchung von Entwicklungen behinderungs- und inklusionsbezogener Einstellungen eignen sich Teilnehmende am FSJ in besonderer Weise durch das Setting des als Bildungsjahr gerahmten Freiwilligenprogramms, das ermöglicht, sowohl praktische Erfahrungen in sozialen Einrichtungen - oftmals in Einrichtungen mit oder für Menschen mit Behinderung - zu sammeln als auch diese pädagogisch begleitet im Seminarkontext zu reflektieren. Auf Basis der skizzierten sozialpsychologischen Einstellungstheorien kann dabei von verschiedenen Faktoren ausgegangen werden, die innerhalb des Freiwilligendienstes auf die Ausbildung bzw. Veränderung von Einstellungen gegenüber Behinderung und Inklusion Einfluss nehmen. Die Kontakthypothese (Allport, 1954) ebenso wie der von Zajonc (1968) erstmal postulierte mere exposure effect legen nahe, dass sich (negative) stereotype Einstellungen gegenüber Menschen mit Behinderung im Laufe des FSJ durch den wiederholten Kontakt insgesamt reduzieren. Da neben der Intensität und der Art des Kontakts insbesondere die subjektive empfundene Kontaktqualität bedingt, inwieweit Einstellungsveränderungen auftreten (z. B. Barr \& Bracchitta, 2012; McManus, Feyes \& Saucier, 2010; Pettigrew, 1998), sind differenzierte Einstellungsentwicklungen der Freiwilligen in Abhängigkeit von der speziellen Ausgestaltung ihres Freiwilligendienstes zu erwarten. Neben diesen Kontaktmerkmalen, die insbesondere von den verschiedenen FSJ-Einsatzstellen abhängen, kann auch von den Seminaren eine einstellungsverändernde Wirkung ausgehen, wenn sie als Möglichkeit zur Reflektion der eigenen Haltung genutzt werden (Maio \& Haddock, 2015).

Ziel des Projektes EFBI ist es, zum einen Einstellungen und Einstellungsentwicklungen von Jugendlichen und jungen Erwachsenen im Verlaufe eines FSJ zu beschreiben und zum anderen mit Einstellungen assoziierte Faktoren sowie Prädiktoren für Einstellungsveränderungen zu identifizieren. Dabei stehen folgende Fragestellungen im Mittelpunkt:

1. Querschnittsanalysen: Mit welchen Faktoren hängen Einstellungen zu Behinderung und Inklusion zusammen?

a. Unterscheiden sich die Einstellungen von Freiwilligen je nach Einsatzbereichen?

b. Wie hängen erfahrungsbezogene (z. B. Vorerfahrungen mit Menschen mit Behinderung) und personenbezogene (z. B. Geschlecht, Motivation für Freiwilligenarbeit) Faktoren mit Einstellungen zusammen?

2. Längsschnittanalysen: (Wie) verändern sich Einstellungen zu Behinderung und Inklusion im Laufe der Teilnahme am FSJ?

a. Unterscheiden sich Veränderungen in den Einstellungen je nach Einsatzbereichen?

b. Entwickeln sich Einstellungen unterschiedlich in Abhängigkeit von erfahrungsbezogenen (z. B. Erfahrungen mit Menschen mit Behinderung in der 
Einsatzstelle) und personenbezogenen Faktoren sowie dem Inhalt der Begleitseminare?

c. Lassen sich empirisch unterscheidbare Typen von Entwicklungsverläufen identifizieren?

\section{A.2 Projektdesign und Stichprobe}

Um die Entwicklung von Einstellungen im Laufe eines FSJ zu untersuchen, wurde die Studie EFBI als Längsschnittstudie mit drei Messzeitpunkten (MZP) konzipiert. Jeweils zu Anfang (MZP1), in der Mitte (MZP2) und am Ende (MZP3) des FSJ (Zyklus 2017/2018) wurden Teilnehmende mit einem standardisierten Fragebogen im Rahmen der verpflichtenden Begleitseminare von zwei niedersächsischen FSJ-Trägern (Internationaler Bund (IB) Göttingen, Internationale Jugendgemeinschaftsdienste (ijgd) Hildesheim) befragt. Zu diesem Zweck wurde auf Basis vorhandener Messinstrumente (u. a. Bosse \& Spörer, 2014; Findler, Vilchinsky \& Werner, 2007; Gebhardt et al., 2011) ein Fragebogen entwickelt, für den einerseits etablierte Skalen adaptiert und andererseits neue Skalen entwickelt wurden, um die entsprechenden Konstrukte breit (d. h. nicht auf eine Behinderungsform und nicht auf den Bereich schulischer Inklusion beschränkt) und der Zielgruppe gemäß zu operationalisieren.

Erfasst wurden somit verschiedene Dimensionen von Einstellungen zu Behinderung und Inklusion sowie Personen- und Kontextvariablen (u. a. Vorerfahrungen mit Menschen mit Behinderung, FSJ-Einsatzstelle, Seminarthemen). Die Befragung war bei einem der Träger als Vollerhebung aller Teilnehmenden angelegt, beim anderen Träger als Teilerhebung. Die Durchführung erfolgte durch geschulte Erhebungsleiter*innen im Rahmen der Begleitseminare, wobei zu den jeweiligen Messzeitpunkten diejenigen Freiwilligen nicht erreicht wurden, die aus gesundheitlichen Gründen oder aufgrund verspäteten Einstiegs bzw. vorzeitigen Ausscheidens aus dem FSJ nicht an den Seminaren teilnahmen. Die Teilnahme an der Befragung erfolgte freiwillig, bis auf wenige Ausnahmen nahmen jeweils alle Freiwilligen der befragten Seminargruppen teil.

Zusätzlich wurde mit einem ausgewählten Sample der Freiwilligen ( $\mathrm{n}=15) \mathrm{im}$ Anschluss an das FSJ narrative Interviews geführt, aus denen mithilfe der Dokumentarischen Methode Orientierungen hinsichtlich Behinderung und Inklusion rekonstruiert werden. Die vorliegende Skalendokumentation bezieht sich nur auf die quantitative Forschungsstudie im Rahmen des Projekts EFBI, der Vollständigkeit halber zeigt die folgende Abbildung (1) aber die zeitliche Gesamtkonzeption des Projekts. 


\begin{tabular}{|c|c|c|c|c|}
\hline & August-Oktober 2017 & Januar-März 2018 & Juni-Juli 2018 & $\begin{array}{l}\text { Oktober- } \\
\text { Dezember } \\
2018\end{array}$ \\
\hline & $\begin{array}{l}\text { Fragebogenerhebung im } \\
\text { Einführungsseminar (MZP1) }\end{array}$ & $\begin{array}{l}\text { Fragebogenerhebung im } \\
\text { Zwischenseminar (MZP2) }\end{array}$ & $\begin{array}{l}\text { Fragebogenerhebung im } \\
\text { Abschlussseminar (MZP3) }\end{array}$ & $\begin{array}{l}\text { Ergänzung } \\
\text { durch } \\
\text { narrative } \\
\text { Interviews }\end{array}$ \\
\hline 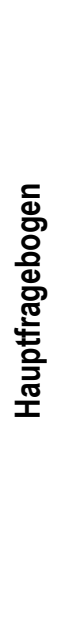 & $\begin{array}{l}\quad \\
\text { Personenbezogene } \\
\text { Variablen } \\
\text { Rahmendaten zum FSJ } \\
\text { Individuelle } \\
\text { Eingangsvoraussetzungen } \\
\text { Einstellungen zu } \\
\text { Behinderung } \\
\text { Einstellungen zu Inklusion } \\
\text { Wissen zu Inklusion } \\
\text { Selbstwirksamkeits- } \\
\text { erwartung }\end{array}$ & $\begin{array}{l}\quad \downarrow \\
\text { Kontextvariablen im FSJ } \\
\text { Einstellungen zu } \\
\text { Behinderung } \\
\text { Einstellungen zu Inklusion } \\
\text { Wissen zu Inklusion } \\
\text { Selbstwirksamkeits- } \\
\text { erwartung }\end{array}$ & $\begin{array}{l}\quad \\
\text { Kontextvariablen im FSJ } \\
\text { Einstellungen zu } \\
\text { Behinderung } \\
\text { Einstellungen zu Inklusion } \\
\text { Wissen zu Inklusion } \\
\text { Selbstwirksamkeits- } \\
\text { erwartung }\end{array}$ & \\
\hline
\end{tabular}

\section{Abb. 1: Gesamtablauf der Datenerbebung}

Die Gesamtstichprobe der Fragebogenstudie besteht aus $n=536$ Befragten. ${ }^{3}$ An der Befragung zum ersten Messzeitpunkt nahmen $n=445$ Freiwillige teil, an der zum zweiten Messzeitpunkt $n=414$ und an der zum dritten $n=344$. Insgesamt nahmen $\mathrm{n}=220 \mathrm{zu}$ allen drei Messzeitpunkten und $\mathrm{n}=427 \mathrm{zu}$ mindestens zwei Messzeitpunkten teil. Einen Überblick über die zum ersten Messzeitpunkt erfassten zentralen Kennwerte der Stichprobe gibt die folgende Tabelle (1) (für Details und Angaben zu denjenigen, die zum zweiten Messzeitpunkt erstmalig teilnahmen, siehe Kapitel 5).

Tab. 1: Deskriptive Stichprobenkennwerte zum ersten Messzeitpunkt

\begin{tabular}{ll}
\hline & 445 \\
Anzahl Teilnehmende & $\hat{O}=151(33.9 \%)$ \\
& $+=275(61.8 \%)$ \\
Alter & $\mathrm{MW}=18.59(\mathrm{SD}=1.40)$ \\
Anzahl Abitur & $297(66.7 \%)$ \\
Anzahl FSJ in Einrichtung für oder mit Menschen mit Behinderung & $281(63.1 \%)$ \\
\hline
\end{tabular}

${ }^{3} \mathrm{Da} 62$ Fälle beim Matching der Befragungsdaten der drei Messzeitpunkte nicht eindeutig zuordbar waren, sind im Längsschnittdatensatz 598 Fälle enthalten. 


\section{A.3 Eingesetzte Fragebögen}

$\mathrm{Zu}$ allen drei Messzeitpunkten füllten die Freiwilligen den Hauptfragebogen aus, der verschiedene Maße zur Erfassung von Einstellungen und Überzeugungen sowie Wissen zu Behinderung und Inklusion enthielt. Die folgende Tabelle (2) gibt Auskunft über die Skalen bzw. Items, die hierfür eingesetzt wurden. ${ }^{4}$

Tab. 2: Übersicht über Variablen zu Einstellungen, Überzeugungen und Wissen

\begin{tabular}{|c|c|c|}
\hline Bereich & Variable & Format \\
\hline \multirow{6}{*}{$\begin{array}{l}\text { Einstellungen zu } \\
\text { Behinderung }\end{array}$} & $\begin{array}{l}\text { Einstellungen zu Behinderung } \\
\text { (7 Dimensionen/Skalen) }\end{array}$ & Itembatterien (Likertskalen) \\
\hline & $\begin{array}{l}\text { Ähnlichkeitseinschätzung von Menschen mit } \\
\text { Behinderung und Menschen ohne Behinderung }\end{array}$ & Grafische Skalen \\
\hline & Soziale Distanz zu Menschen mit Behinderung & Guttman-Skalen \\
\hline & $\begin{array}{l}\text { Reaktion auf Körperbehinderung (kognitiv, } \\
\text { affektiv, behavioral) }\end{array}$ & $\begin{array}{l}\text { Vignette mit Itembatterien (Likertska- } \\
\text { len) bzw. semantischem Differenzial }\end{array}$ \\
\hline & Eigenes Verständnis von Behinderung & Offene Frage \\
\hline & $\begin{array}{l}\text { Frage nach Einstellungsveränderungen zu Be- } \\
\text { hinderung }\end{array}$ & $\begin{array}{l}\text { Dichotom (ja/nein) mit anschließender } \\
\text { offener Frage (nur MZP2 und MZP3) }\end{array}$ \\
\hline \multirow{3}{*}{$\begin{array}{l}\text { Einstellungen zu } \\
\text { Inklusion }\end{array}$} & $\begin{array}{l}\text { Befürwortung von Inklusion im Sinne der UN- } \\
\text { Behindertenrechtskonvention } \\
\text { (4 Dimensionen/Skalen) }\end{array}$ & Itembatterien (Likertskalen) \\
\hline & $\begin{array}{l}\text { Befürwortung von schulischer Inklusion bei } \\
\text { verschiedenen Behinderungsformen } \\
\text { (3 Dimensionen/Skalen) }\end{array}$ & Itembatterien (Likertskalen) \\
\hline & $\begin{array}{l}\text { Befürwortung von schulischer Inklusion bei } \\
\text { einer spezifischen Behinderung }\end{array}$ & $\begin{array}{l}\text { Vignette mit offenen und geschlosse- } \\
\text { nen Fragen }\end{array}$ \\
\hline $\begin{array}{l}\text { Selbstwirksamkeits- } \\
\text { erwartung }\end{array}$ & $\begin{array}{l}\text { Selbstwirksamkeitserwartung in der Arbeit mit } \\
\text { Menschen mit Behinderung }\end{array}$ & Itembatterie (Likertskala) \\
\hline \multirow{3}{*}{$\begin{array}{l}\text { Wissen zu Inklusi- } \\
\text { on }\end{array}$} & Eigenes Verständnis von Inklusion & $\begin{array}{l}\text { Dichotom (ja/nein) mit anschließender } \\
\text { offener Frage }\end{array}$ \\
\hline & $\begin{array}{l}\text { Kenntnis der UN-Behindertenrechtskonvention } \\
\text { (Selbsteinschätzung) }\end{array}$ & Dichotom (ja/nein) \\
\hline & $\begin{array}{l}\text { Kenntnis von Modellen Exklusion, Separation, } \\
\text { Integration, Inklusion (Testaufgabe) }\end{array}$ & Zuordnungsaufgabe \\
\hline
\end{tabular}

Anmerkungen. Soweit nicht anders vermerkt, wurden die hier dargestellten Skalen/Items im Hauptfragebogen zu allen Messzeitpunkten in identischer Form vorgelegt.

${ }^{4}$ Die Antworten auf die offenen Fragen sind nicht in dieser Skalendokumentation enthalten. 
Zur Erfassung personen- und kontextbezogener Variablen enthielt der Hauptfragebogen weitere Items, über die die folgende Tabelle (3) eine Übersicht bietet. Dabei waren alle Fragen zu demographischen Angaben, zur FSJ-Einsatzstelle sowie zu den Eingangsvoraussetzungen zum FSJ nur zum ersten Messzeitpunkt im Hauptfragebogen enthalten. $\mathrm{Zu}$ Messzeitpunkt 2 und 3 wurden diese Angaben von denjenigen, die zu diesem Zeitpunkt zum ersten Mal teilnahmen, über den Zusatzbogen Erstteilnahme erfasst. Alle diejenigen, die während des FSJ die Einsatzstelle wechselten, füllten zum jeweils nächsten Messzeitpunkt den Zusatzbogen Einsatzstellenwechsel aus, der Fragen zu Gründen des Wechsels und Fragen zur neuen Einsatzstelle enthielt. Aufgrund der geringen Fallzahl ( $n=7)$ werden die Angaben aus dem Zusatzbogen Einsatzstellenwechsel in dieser Skalendokumentation nicht aufgeführt.

Um die in den Seminaren behandelten Inhalte als Kontextvariablen einzubeziehen, wurde ein Zusatzbogen Seminarthemen eingesetzt, der Fragen zu den Schwerpunkten des Seminars, den eingesetzten Methoden und den angestrebten Lernzielen enthielt. Nach Möglichkeit wurde dieser von den jeweiligen Seminarleitungen ausgefüllt. Da die Zusammensetzung der Seminargruppen ebenso wie die Zuordnung zu Seminarleitungen je nach pädagogischem Konzept des Trägers im Laufe des Jahres wechselte, wurden teilweise statt der Seminarleitungen die Freiwilligen selbst zu den Seminaren befragt. Die Inhalte des Zusatzbogens Seminarthemen werden der Vollständigkeit halber in Tabelle 3 aufgeführt, sind darüber hinaus aber nicht Bestandteil der vorliegenden Skalendokumentation.

Tab. 3: Übersicht über personen- und kontextbezogene Variablen

\begin{tabular}{lll}
\hline Bereich & Variable/Item & Enthalten in Fragebogen \\
\hline $\begin{array}{l}\text { Personenbezogene } \\
\text { Variablen }\end{array}$ & $\begin{array}{l}\text { Demographische Angaben (Alter, Geschlecht, } \\
\text { Schulabschluss, besuchte Schulform) }\end{array}$ & $\begin{array}{l}\text { Hauptfragebogen MZP1, Zusatz- } \\
\text { bogen Erstteilnahme MZP2 und } \\
\text { MZP3 }\end{array}$ \\
\hline $\begin{array}{l}\text { Rahmendaten zum } \\
\text { FSJ }\end{array}$ & Einsatzstelle, erster Arbeitstag & $\begin{array}{l}\text { Hauptfragebogen MZP1, Zusatz- } \\
\text { bogen Erstteilnahme MZP2 und } \\
\text { MZP3, Zusatzbogen Einsatzstel- } \\
\text { lenwechsel MZP2 und MZP3 }\end{array}$ \\
\hline & $\begin{array}{l}\text { Vorerfahrungen mit Menschen mit Behinderung } \\
\text { (Quantität und Valenz) }\end{array}$ & $\begin{array}{l}\text { Hauptfragebogen MZP1, Zusatz- } \\
\text { bogen Erstteilnahme MZP2 und } \\
\text { MZP3 }\end{array}$ \\
$\begin{array}{ll}\text { Individuelle Ein- } \\
\text { gangs- } \\
\text { voraussetzungen }\end{array}$ & $\begin{array}{l}\text { Motivation zum FSJ } \\
\text { Houptfragebogen MZP1, Zusatz- } \\
\text { bogen Erstteilnahme MZP2 und } \\
\text { MZP3 }\end{array}$ & $\begin{array}{l}\text { Hauptfragebogen MZP1, Zusatz- } \\
\text { bogen Erstteilnahme MZP2 und } \\
\text { MZP3, Zusatzbogen Einsatzstel- } \\
\text { lenwechsel MZP2 und MZP3 }\end{array}$ \\
& $\begin{array}{l}\text { Gründe für Wechsel der Einsatzstelle } \\
\text { Nur Zusatzbogen Einsatzstellen- } \\
\text { wechsel MZP2 und MZP3 }\end{array}$
\end{tabular}




\begin{tabular}{lll}
\hline Bereich & Variable/Item & Enthalten in Fragebogen \\
\hline & $\begin{array}{l}\text { Erfahrungen mit Menschen mit Behinderung } \\
\text { (Quantität und Valenz) }\end{array}$ & $\begin{array}{l}\text { Hauptfragebogen MZP2 und } \\
\text { MZP3 }\end{array}$ \\
$\begin{array}{l}\text { Kontextvariablen im } \\
\text { FSJ }\end{array}$ & $\begin{array}{l}\text { Inhalte der Begleitseminare (Themen, Methoden, } \\
\text { Lernziele) }\end{array}$ & $\begin{array}{l}\text { Fragen im Hauptfragebogen } \\
\text { MZP1, MZP2 und MZP3 integriert } \\
\text { oder als Zusatzbogen Seminarthe- } \\
\text { men MZP1, MZP2 und MZP3 von } \\
\text { den Seminarleitungen ausgefüllt }\end{array}$ \\
\hline
\end{tabular}

\section{A.4 Das Team}

\section{Wissenschaftliche Leitung}

Prof. Dr. Ariane S. Willems

\section{Wissenschaftliche Mitarbeiterin und Doktorandin}

Karina Meyer, M. Ed. (seit 7/2017)

\section{Kooperationspartnerin (Qualitative Ergänzungsstudie)}

Dr. Catharina I. Keßler

\section{Studentische Mitarbeiter*innen}

Gwendolyn Barthe

Lena Heitkamp

Melissa Eckert

Mareike Islar

Daniel Erdmann

Moritz Frechen

Kim Göwecke

Steffen Berke
Henrike Sannecke

Magdalena Schütz

Sofie Thielke

Philine Thomasius

Lotte Wegener

An der erfolgreichen Vorbereitung, Durchführung und Auswertung des Projekts EFBI war eine Vielzahl von Personen beteiligt, denen wir an dieser Stelle herzlich danken möchten. Unser besonderer Dank gilt den beiden FSJ-Trägern Internationaler Bund (IB) Freiwilligendienste Göttingen und Internationale Jugendgemeinschaftsdienste (ijgd) Landesvereine Niedersachsen e. V., die mit ihrer freiwilligen Teilnahme an den Befragungen die Realisierung des Projekts EFBI ermöglicht haben. Insbesondere möchten wir uns bei Jutta Plümer und Jana Postels (ijgd Hildesheim) und dem pädagogischen Team des IB Göttingen bedanken, die uns mit ihrem Engagement bei der Koordination der Datenerhebungen unterstützt haben, sowie bei allen pädagogi- 
schen Mitarbeiter*innen und Honorarkräften beider Träger, die uns die Befragung in ihren Seminaren ermöglicht haben. Nicht zuletzt wäre das Projekt EFBI nicht möglich gewesen ohne die Bereitschaft der FSJler*innen selbst, wiederholt unsere Fragebögen zu bearbeiten und uns von ihren Erfahrungen im Freiwilligendienst zu berichten. An dieser Stelle möchten wir deshalb allen Teilnehmenden unseren herzlichen Dank aussprechen.

\section{A.5 Publikationen, Vorträge und Qualifikationsarbeiten}

\section{Publikationen (Auswahl)}

Meyer, K. \& Willems, A. S. (i. E.). Verändern sich Einstellungen zu Behinderung durch Kontakterfahrungen und Bildungsseminare? Eine quantitative Längsschnittanalyse zu Freiwilligendiensten. In S. Fränkel, M. Grünke, T. Hennemann, D. Hövel, C. Melzer \& K. Ziemen, S. Fränkel, M. (Hrsg.), Teilhabe in allen Lebensbereichen? Ein Blick zurück und nach vorn. Bad Heilbrunn: Klinkhardt.

Meyer, K. \& Willems, A. S. (i. E.). Verändern sich Einstellungen zu Behinderung im Verlauf von Freiwilligendiensten? Eine Längsschnittanalyse latenter Profile. In A. S. Willems (Hrsg.), ,Warum und Wozu?" Motivation und Einstellung in (außer)schulischen Bildungsprozessen. Göttingen: Universitätsverlag (Erziehungswissenschaftliche Schriften).

Frechen, M., Meyer, K. \& Willems, A. S. (i. E.). Wie verändern Kontakt und Kontakt(un-)sicherheit Einstellungen gegenüber Menschen mit Behinderung? Befunde einer Studie zum Freiwilligen Sozialen Jahr (EFBI). In A. S. Willems (Hrsg.), „Warum und Wozu?" Motivation und Einstellung in (außer)schulischen Bildungsprozessen. Göttingen: Universitätsverlag (Erziehungswissenschaftliche Schriften).

Meyer, K., Schröter, A. \& Bierschwale, C. (2021). Behinderung als Differenzkategorie in der quantitativen Einstellungsforschung - eine kritische Auseinandersetzung mit standardisierten Messinstrumenten. In S. Gabriel, P. Leinhos, K. Kotzyba, D. Matthes, K. Meyer \& M. Völcker (Hrsg.), Soziale Differenz und Reifizizerung. Theoretische Zugänge und forschungspraktische Bearbeitungen (Studien zur Schul- und Bildungsforschung (SZSBF)) (S. 101-134). Wiesbaden: Springer VS.

Willems, A. S. \& Meyer, K. (2019). Welche Rolle spielen Einflussfaktoren vor und während des Studiums für die Überzeugungen von Lehramtsstudierenden zu schulischer Inklusion? In T. Ehmke, P. Kuhl \& M. Pietsch (Hrsg.). Lebrer. Bildung. Gestalten. Beiträge zur empirischen Forschung in der Lebrerbildung (S. 71-84). Weinheim: Beltz Juventa.

\section{Konferenzbeiträge (Auswahl)}

Meyer, K. \& Willems, A. S. (2021). Einstellungen zu Behinderung im Laufe von Freiwilligendiensten - eine quantitative Längsschnittstudie. Vortrag bei der Thementagung (online) „Inklusion und Bildung als Thema der empirischen Bildungsfor- 
schung: Theoretische Ansätze und empirische Befunde“ der Gesellschaft für Empirische Bildungsforschung (GEBF).

Meyer, K. \& Willems, A. S. (2021). How do perspectives on disability change during one year of volunteering? An analysis of latent growth curves. Vortrag bei der European Conference (online) on Educational Research (ECER), Genova.

Frechen, M., Meyer, K. \& Willems, A. S. (2020). Wie verändern Kontakt und Kontakt(un)sicherheit Einstellungen gegenüber Menschen mit Behinderung? Befunde einer Studie zum Freiwilligen Sozialen Jahr (EFBI). Vortrag bei der Herbsttagung (online) der Arbeitsgruppe Empirische Sonderpädagogische Forschung (AESF), Potsdam.

Meyer, K. \& Willems, A. S. (2020). Einstellungen zu Behinderung und Inklusion im Kontext von Freiwilligendiensten - eine quantitative Längsschnittstudie. Vortrag bei der Tagung (online) der DGfE-Sektion Sonderpädagogik, Köln.

Willems, A. S. \& Meyer, K. (2019). Inklusive Einstellungen von Lehramtsstudierenden - das Zusammenspiel von Einflussfaktoren vor und im Studium. Symposiumsbeitrag bei der Tagung der DGfE-Sektion Sonderpädagogik, Wuppertal.

Meyer, K., Keßler, C. I. \& Willems, A. S. (2019). Einstellungen zu Behinderung im Laufe von Freiwilligendiensten - eine methodenplurale Längsschnittstudie. Symposiumsbeitrag bei der Tagung der Arbeitsgruppe für Empirische Pädagogische Forschung (AEPF), Münster.

Meyer, K. \& Willems, A. S. (2019). How do experiences prior and during teacher education influence pro-inclusive attitudes of pre-service teachers? Vortrag bei der European Conference on Educational Research (ECER), Hamburg.

Meyer, K. \& Willems, A. S. (2019). Überzeugungen von Lehramtsstudierenden zu Inklusion: Einflussfaktoren vor und im Studium. Poster beim Forschungstag des Zentrums für empirische Unterrichts- und Schulforschung (ZeUS), Göttingen.

Meyer, K., Erdmann, D. \& Willems, A. S. (2018). Einstellungen zu Behinderung und Inklusion: Testung eines mehrfaktoriellen Messinstruments. Poster bei der Tagung der Arbeitsgruppe für Empirische Pädagogische Forschung (AEPF), Lüneburg.

Meyer, K., Erdmann, D. \& Willems, A. S. (2018). Attitudes towards disability and inclusion: Results from a longitudinal study on young volunteers working with people with a disability. Vortrag bei der European Association for Research on Learning and Instruction (EARLI), Special Interest Group (SIG) 15 - Special Educational Needs, Potsdam.

Meyer, K., Erdmann, D. \& Willems, A. S. (2018). Attitudes towards disability and inclusion: Results from a longitudinal study on young volunteers working with people with a disability. Vortrag bei der European Conference on Educational Research (ECER), Bolzano. 


\section{Qualifikationsarbeiten (Auswahl)}

\section{Dissertation}

Karina Meyer, M. Ed. (laufend). Perspektiven auf Inklusion in der empirischen Bildungsforschung [Arbeitstitel]. Georg-August-Universität Göttingen, Fachbereich Erziehungswissenschaft.

\section{Abscblussarbeiten}

Veramaria Merten (2021). Wie entwickelt sich bei jungen Freiwilligen die subjektive Definition von ,Behinderung ${ }^{6}$ im Laufe ihres Freiwilligen Sozialen Jahres? Eine empirische Analyse von Definitionen und Korrelationen mit personen- und kontextbezogenen Merkmalen. Georg-August-Universität Göttingen, Master of Education.

Ann-Kathrin Prinz (2021). Wie definieren junge Freiwillige eine inklusive Gesellschaft? Eine empirische Analyse zur Begriffsbestimmung von Inklusion und Zusammenhängen mit personen- und kontaktbezogenen Merkmalen. Georg-AugustUniversität Göttingen, Master of Education.

Moritz Frechen (2020). Einstellungen von jungen Freiwilligen zu Behinderung und Inklusion - eine quantitative längsschnittliche Analyse. Georg-August-Universität Göttingen, Master Erziehungswissenschaft.

Anna-Lina Schiller (2020). Wie definieren junge Freiwillige ,Behinderung? Eine empirische Analyse von Begriffsbestimmungen und Zusammenhängen mit personenund kontextbezogenen Merkmalen. Georg-August-Universität Göttingen, Master of Education.

Marie-Sophie Janßen (2019). Eine empirische Untersuchung über den Einfluss von Motivation, Vorerfahrung und Geschlecht auf die Einstellung zu schulischer Inklusion von Lehramtsstudierenden. Georg-August-Universität Göttingen, Master of Education.

Sandro, Czaia (2019). Einstellung zu schulischer Inklusion - eine empirische Untersuchung von Einflussfaktoren. Georg-August-Universität Göttingen, Master of Education.

Fabian Probst (2019). Identitätsarbeit von jungen Erwachsenen während des Freiwilligen Soziales Jahres (FSJ) - eine qualitative Interviewstudie. Georg-AugustUniversität Göttingen, Master of Education.

Lea Holdmann (2018). Perspektiven von FSJler*innen auf Menschen mit Behinderungen - eine empirische Fallanalyse. Georg-August-Universität Göttingen, Master of Education.

Mareike Andrea Schmidt (2018). FSJ als Übergangspfad zwischen Schule und Beruf/Studium. Offene Fragen für die qualitative Sozialforschung. Georg-AugustUniversität Göttingen, Bachelor Sozialwissenschaften. 


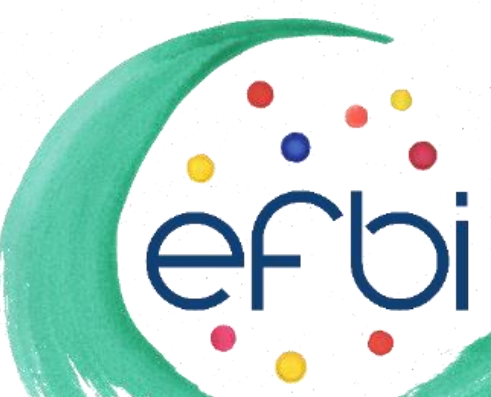

SKALENDOKUMENTATION 


\section{B Hinweise zur Skalendokumentation EFBI}

\section{B.1 Aufbau der Skalendokumentation}

Der vorliegende Band systematisiert und dokumentiert die im Projekt EFBI - Einstellungen von FSJler*innen zu Behinderung und Inklusion entwickelten und eingesetzten Items und Skalen des Hauptfragebogens, den die Absolvierenden des FSJ ausfüllten. ${ }^{5}$ Neben den projekteigenen Konstrukten wurden in EFBI auch etablierte Skalen u. a. zur Erfassung der Selbstwirksamkeitserwartung weiterentwickelt bzw. für den gegebenen Studienkontext adaptiert. Die Skalendokumentation wendet sich damit in erster Linie an Wissenschaftler*innen, die an den Details der Erhebungsinstrumente, der theoretischen Verankerung der untersuchten Konstrukte sowie der methodischen Aufbereitung der Daten (u. a. Verfahren zur Skalierung der Fragebogendaten) interessiert sind.

Inhaltlich gliedert sich die Dokumentation der Erhebungsinstrumente in drei Teilbereiche: ${ }^{6}$

1. In Abschnitt 1 werden die in EFBI entwickelten Items und Skalen zur Erfassung von Einstellungen $z u$ Behinderung (Einstellungen $\mathrm{zu}$ Behinderung, Ingroup/Outgroup-Wahrnehmung, Soziale Distanz, Reaktionen auf Körperbehinderung) sowie deren Kennwerte dargestellt.

2. In Abschnitt 2 werden die entsprechenden Informationen für Einstellungen $q u$ Inklusion (Befürwortung von Inklusion im Sinne der UN-

\footnotetext{
${ }^{5}$ Neben dem hier berichteten Hauptfragebogen kamen Zusatzbögen für Freiwillige zum Einsatz, die erst zu einem späteren Zeitpunkt erstmalig teilnahmen oder die zwischenzeitlich die Einsatzstelle gewechselt hatten, sowie Zusatzbögen zur Erfassung der Themen, die in den Begleitseminaren behandelt wurden (siehe Kapitel A.3).

${ }^{6}$ Die Reihenfolge der Konstrukte in der vorliegenden Skalendokumentation weicht an einigen Stellen von der Reihenfolge der Konstrukte im Fragebogen ab.
} 
Behindertenrechtskonvention, Befürwortung schulischer Inklusion, Befürwortung von Inklusion bei spezifischer Behinderung) berichtet.

3. In Abschnitt 3 werden die Kennwerte für Selbstwirksamkeitserwartung der Freiwilligen in der Arbeit mit Menschen mit Behinderung erfasst.

4. In Abschnitt 4 werden Items dargestellt, die Wissen zu Inklusion erheben.

5. In Abschnitt 5 werden schließlich Items berichtet, die näheren Aufschluss über die Stichprobe geben (personenbezogene Angaben, Rahmendaten zum Freiwilligendienst, individuelle Eingangsvoraussetzungen und Kontextvariablen im Freiwilligendienst).

Für alle in EFBI berücksichtigten Konstrukte werden die Angaben in dieser Skalendokumentation systematisch für alle drei Messzeitpunkte aufgeführt. Die Darstellung folgt einem einheitlichen Muster, nach dem für jedes Konstrukt zwei Tabellen mit ausführlichen inhaltlichen und methodischen Informationen dargestellt werden.

Zunächst erfolgt für jedes Konstrukt eine kurze Übersicht mit folgenden Informationen:

- Variablenname: Kurzbezeichnung der Skala im Datensatz. Skalen, die basierend auf den Daten zu MZP1 gebildet wurden, tragen im Variablennamen das Präfix $x a$. Skalen, die basierend auf den Daten zu MZP2 gebildet wurden, das Präfix $x b$ und Skalen, die auf Daten zu MZP3 basieren, das Präfix xc.

- Instruktion: Wortlaut der Instruktion zur Bearbeitung der Items im Fragebogen.

- Quelle und weiterführende Literatur: Angabe zur Herkunft der Items (falls zutreffend) sowie zu weiterführender, theoretisch-konzeptueller oder empirischer Literatur.

- Skalierung der Items: Angaben zu den Wertelabels/Antwortkategorien der Items. Items, die Teil einer Skala sind, weisen in der Regel ein sechsstufiges Antwortformat mit Ausprägungen von $1=$, stimme überhaupt nicht $\mathrm{zu}^{6}$ bis $6=$ ,stimme voll $\mathrm{zu}^{6}$ (ohne Beschriftung der Zwischenstufen) auf, sodass hohe Werte einer hohen Zustimmung der Befragten auf den Items entsprechen (unabhängig von der Formulierung der Items).

- Inverse Items: An dieser Stelle werden die Itembezeichnungen der Items aufgeführt, die nicht in Schlüsselrichtung der intendierten Gesamtskala formuliert sind und deren Wertelabels entsprechend für die Skalenbildung umgepolt (rekodiert) wurden.

- Anzahl Items: Angabe zur Anzahl Items, die in die Skalenbildung eingegangen sind.

- Skalenbildung: Die Skalen werden im Anschluss an die Item- und Skalenanalyse in der Regel durch die Berechnung des arithmetischen Mittels (MW) der Ausprägungen auf den Einzeltems gebildet.

Im Anschluss an diese Kurzübersicht folgen für jedes Konstrukt zwei Tabellen.

1. In der ersten Tabelle sind die Itembezeichnungen (Kürzel) der Items für MZP1, MZP2 und MZP3 sowie der Itemwortlaut aufgeführt. 
2. In der zweiten Tabelle werden schließlich die statistischen Kennwerte (Mittelwert (MW), Standardabweichung (SD), Trennschärfe ( $\mathrm{r}_{\mathrm{it}}$ ), standardisierte Faktorladungen $\left(\lambda_{\mathrm{ij}}\right)$; vgl. Abschnitt B.2) auf Item- und Skalenebene dargestellt.

In Bezug auf die Itembezeichnungen kann es in diesen beiden Tabellen zu Abweichungen kommen: Während in der ersten Tabelle die Originalbezeichnungen der Items aufgeführt sind, werden in der zweiten Tabelle die für die Skalenbildung rekodierten Items mit ihren eigenen Itembezeichnungen ausgewiesen. Die Bezeichnungen für rekodierte Items setzen sich dabei immer aus dem Präfix $r$ und den Originalbezeichnungen der Items zusammen. Die Itemkennwerte werden in diesen Tabellen für die bereits rekodierten Items ausgewiesen.

Im Folgenden werden die Verfahren beschrieben, mit denen die Daten von EFBI faktoranalytisch geprüft und auf Basis deren Resultate die Skalenzusammenfassungen erfolgten. Insbesondere wird dazu auf die durchgeführten ein- und mehrfaktoriellen konfirmatorischen Faktorenanalysen (Moosbrugger \& Schermelleh-Engel, 2012) sowie auf die Reliabilitätsanalysen (Moosbrugger \& Kelava, 2012; Kuckartz, Rädiker, Ebert \& Schehl, 2013; Schermelleh-Engel \& Werner, 2012) eingegangen.

\section{B.2 Methodische Grundlagen der Item- und Skalenanalysen}

Die Skalierung der Fragebogenitems erfolgt in zwei Schritten auf Basis der Annahmen der Klassischen Testtheorie (Moosbrugger, 2012).

1. Die Überprüfung der Dimensionalität (Faktorstruktur) der Items erfolgt dabei über die Anwendung von konfirmatorischen Faktorenanalysen unter Verwendung der Software Mplus 8.4 (Muthén \& Muthén, 2017). Je nach theoretischkonzeptuellen Annahmen, die der Itemkonstruktion zu Grunde lagen, wurden einfaktorielle und/oder mehrfaktorielle Faktorenanalysen durchgeführt. In der vorliegenden Skalendokumentation werden basierend auf den Befunden der Faktorenanalysen für die Einzelitems einer Skala die standardisierten Faktorladungen $\lambda_{\mathrm{ij}}$ inklusive ihres Signifikanzniveaus berichtet. In einem ersten Schritt werden dazu für jedes Konstrukt die einfaktoriellen Messmodelle berichtet. Falls zutreffend werden im Anschluss daran die Befunde der mehrfaktoriellen Faktorenanalysen dargestellt. In diesem Fall werden neben den standardisierten Faktorladungen der Einzelitems auch die latenten Interkorrelationen zwischen den Faktoren (inklusive ihres Signifikanzniveaus) ausgewiesen. Schließlich werden für alle Mess- und Strukturmodelle die entsprechenden Modellgütekriterien angegeben (siehe unten).

2. Ergänzend zu den konfirmatorischen Faktorenanalysen wurden in EFBI anschließend klassische Reliabilitätsanalysen unter Verwendung der Software SPSS 26 (Bühl, 2018) durchgeführt. In der vorliegenden Skalendokumentation werden basierend auf diesen Item- und Skalenanalysen die deskriptiven Kennwerte (MW/SD) der Einzelitems und ihre Trennschärfen ( $\mathrm{r}_{\mathrm{it}}$ ) angegeben. Auf Skalenebene wird schließlich als $\mathrm{Maß}$ der internen Konsistenz der Skalen das Cronbachs Alpha - basierend auf den latenten Inter-Item-Korrelationen - für al- 
le Konstrukte dargestellt. Zudem werden die deskriptiven Kennwerte (MW/SD) auf Skalenebene ausgewiesen.

Die konfirmatorischen Faktorenanalysen wurden mit folgenden methodischen Spezifizierungen durchgeführt:

- Skalierung der Items: Die in der Regel sechsstufig skalierten Items mit Endpolbeschriftung werden in den ein- und mehrfaktoriellen Faktorenanalysen als intervallskalierte Items modelliert (Muthén \& Muthén, 2017).

- Umgang mit fehlenden Werten: Um fehlende Werte (item non-response) angemessen zu berücksichtigen, werden die Modellparameter in den ein- und mehrfaktoriellen Faktorenanalysen unter Verwendung des Full-Information-MaximumLikelihood-Algorithmus (FIML) geschätzt (Muthén \& Muthén, 2017).

Zur Beurteilung der Modellpassung im Rahmen der konfirmatorischen Faktorenanalysen können unterschiedliche (i) absolute und (ii) inkrementelle Fit Indices genutzt werden (u. a. Eid, 1999; Hooper, Coughlan \& Mullen, 2008; Hu \& Bentler, 1999; Kline, 2011; Marsh, Hau \& Wen, 2004; Schermelleh-Engel, Moosbrugger \& Müller, 2003; Schreiber, Nora, Stage, Barlow \& King, 2006; Sivo, Fan, Witta \& Willse, 2006; West, Taylor \& Wu, 2012).

- In der vorliegenden Skalendokumentation berichten wir für alle ein- und mehrfaktoriellen Messmodelle als Maß des absoluten Modellfits (Goodness-of-Fit) den Wert des $\chi^{2}$-Anpassungstests, wobei ein signifikanter $\chi^{2}$-Wert auf eine signifikante Abweichung zwischen der durch das Modell implizierten Kovarianzmatrix und der empirisch beobachteten Kovarianz hinweist. Da der $\chi^{2}$-Wert bei großen Stichproben bereits triviale Unterschiede zwischen der durch das Modell implizierten Kovarianzmatrix und der empirisch beobachteten Kovarianz als signifikant ausweist, sollte zur Beurteilung der Modellgüte in erster Linie der Quotient aus $\chi^{2}$-Wert und den modellspezifischen Freiheitsgraden $\left(\chi^{2} / \mathrm{df}\right)$ genutzt werden.

- Als inkrementelle Fitindizes werden der Comparative Fit Index (CFI) sowie der Tucker-Lewis Index (TLI) berichtet. Für die Berechnung beider Indizes wird das jeweils angenommene Modell mit dem stärker restriktiveren Nullmodell (Baseline-Model) verglichen, wobei der Unterschied beider Modelle möglichst groß ausfallen sollte. Schließlich werden als sogenannte Badness-of-Fit Indices der Root Mean Square Error of Approximation (RMSEA), der robust gegenüber dem Stichprobenumfang ist und zudem die Sparsamkeit der Modelle berücksichtigt, sowie der Wert des (nicht-stichprobensensitiven) Standardized Root Mean Square Residual (SRMR) berichtet.

Mittlerweile existieren eine Vielzahl von Simulationsstudien, auf deren Basis cut-offWerte zur Interpretation der einzelnen Fit Indices formuliert wurden (zsfd. Hu \& Bentler, 1999; Schermelleh-Engel, Moosbrugger \& Müller, 2003; Schreiber, Nora, Stage, Barlow \& King, 2006; Sivo, Fan, Witta \& Willse, 2006) - wobei die jeweiligen Empfehlungen je nach Publikation leicht variieren. Zur Bewertung der vorliegenden Messmodelle können folgende Kriterien herangezogen werden: 
Tab. 4: Cut-off-Werte zur Beurteilung der Modellgüte von konfirmatorischen Faktorenanalysen

\begin{tabular}{lll}
\hline \multirow{2}{*}{ Fit Index } & \multicolumn{1}{c}{ Hohe Modellgüte } & Akzeptable Modellgüte \\
\cline { 2 - 3 } & Cut-off-Wert & Cut-off-Wert \\
\hline$\chi^{2 / \mathrm{df}}$ & $0 \leq \chi^{2} / \mathrm{df} \leq 2$ & $2<\chi 2 / \mathrm{df} \leq 3$ \\
CFI & $.97 \leq \mathrm{CFI} \leq 1.00$ & $.95 \leq \mathrm{CFI}<.97$ \\
TLI & $.97 \leq \mathrm{TLI} \leq 1.00$ & $.95 \leq \mathrm{TLI}<.97$ \\
RMSEA & $0 \leq$ RMSEA $\leq .05$ & $.05<$ RMSEA $\leq .08$ \\
SRMR & $0 \leq$ SRMR $\leq .05$ & $.05<$ SRMR $\leq .10$ \\
\hline
\end{tabular}




\section{Einstellungen zu Behinderung}

\subsection{Einstellungen zu (Menschen mit) Behinderung}

\subsubsection{Menschen mit Behinderung als Bereicherung}

Kurzbezeichnung Variablenname

Instruktion

Quelle

Weiterführende Literatur

Skalierung (Antwortformat)

Inverse Items (-)

Anzahl Items

Skalenbildung

Instrument xa_eb_br/xb_eb_br/xc_eb_br

(xa_ebi_Ber/xb_ebi_Ber/xc_ebi_Ber)

Es folgt nun eine Reihe von verschiedenen Meinungen und möglichen Reaktionen zu Menschen mit Behinderung. Bitte gibt zu jeder Aussage an, inwieweit du ihr zustimmst.

Eigenentwicklung

Cloerkes, 2007; Kastl, 2017; Watson, Roulstone \& Thomas, 2014

Endpolbeschriftung; 1 = stimme überhaupt nicht zu, $(\ldots), 6=$ stimme voll $\mathrm{zu}$

4

per Mittelwert

Hauptfragebogen Messzeitpunkt 1, 2 und 3

\begin{tabular}{|c|c|c|c|}
\hline MZP1 & MZP2 & MZP3 & Itemwortlaut \\
\hline a_eb35 & b_eb35 & c_eb35 & $\begin{array}{l}\text { Menschen mit Behinderung können einige Dinge besser als Menschen ohne } \\
\text { Behinderung. }\end{array}$ \\
\hline a_eb37 & b_eb37 & c_eb37 & Menschen mit Behinderung bereichern die Gesellschaft. \\
\hline a_eb43 & b_eb43 & c_eb43 & $\begin{array}{l}\text { Menschen mit Behinderung leisten einen positiven Beitrag zur Weiterent- } \\
\text { wicklung unserer Gesellschaft. }\end{array}$ \\
\hline a_eb47 & b_eb47 & c_eb47 & $\begin{array}{l}\text { Menschen ohne Behinderung können von Menschen mit Behinderung viel } \\
\text { lernen. }\end{array}$ \\
\hline
\end{tabular}




\begin{tabular}{|c|c|c|c|c|c|c|c|c|c|c|c|c|c|c|}
\hline \multirow{2}{*}{\multicolumn{3}{|c|}{ Itembezeichnung }} & \multicolumn{12}{|c|}{ Itemkennwerte } \\
\hline & & & \multicolumn{4}{|c|}{ MZP1 } & \multicolumn{4}{|c|}{ MZP2 } & \multicolumn{4}{|c|}{ MZP3 } \\
\hline MZP1 & MZP2 & MZP3 & MW & $\mathrm{SD}$ & $\mathrm{r}_{\mathrm{it}}$ & $\lambda_{\mathrm{ij}}$ & MW & $\mathrm{SD}$ & $r_{i t}$ & $\lambda_{\mathrm{ij}}$ & MW & SD & $\mathrm{r}_{\mathrm{it}}$ & $\lambda_{\mathrm{ij}}$ \\
\hline a_eb35 & b_eb35 & c_eb35 & 4.86 & 1.26 & .47 & $.52 * *$ & 4.86 & 1.18 & .47 & $.52 * *$ & 4.91 & 1.26 & .59 & $.64 * *$ \\
\hline a_eb37 & b_eb37 & c_eb37 & 4.74 & 1.23 & .58 & $.77 * *$ & 4.75 & 1.23 & .63 & $.83^{* *}$ & 4.91 & 1.14 & .69 & $.90 * *$ \\
\hline a_eb43 & b_eb43 & c_eb43 & 4.75 & 1.13 & .63 & $.74 * *$ & 4.78 & 1.15 & .60 & $.68^{* *}$ & 4.83 & 1.22 & .64 & $.70^{* *}$ \\
\hline a_eb47 & b_eb47 & c_eb47 & 4.96 & 1.11 & .53 & $.68 * *$ & 4.87 & 1.19 & .57 & $.76^{* *}$ & 4.85 & 1.12 & .61 & $.78^{* *}$ \\
\hline \multirow{4}{*}{\multicolumn{3}{|c|}{ Skalenkennwerte }} & \multicolumn{2}{|c|}{ Cronbachs $\alpha$} & \multicolumn{2}{|l|}{.76} & \multicolumn{2}{|c|}{ Cronbachs $\alpha$} & \multicolumn{2}{|l|}{.77} & \multicolumn{2}{|c|}{ Cronbachs $\alpha$} & \multicolumn{2}{|l|}{.82} \\
\hline & & & \multicolumn{2}{|c|}{ MW } & \multicolumn{2}{|l|}{4.82} & \multicolumn{2}{|l|}{ MW } & \multicolumn{2}{|l|}{4.80} & \multicolumn{2}{|l|}{ MW } & \multicolumn{2}{|c|}{4.82} \\
\hline & & & \multicolumn{2}{|l|}{ SD } & \multicolumn{2}{|l|}{.90} & \multicolumn{2}{|l|}{$\mathrm{SD}$} & \multicolumn{2}{|l|}{.92} & \multicolumn{2}{|l|}{$\mathrm{SD}$} & \multicolumn{2}{|l|}{1.01} \\
\hline & & & \multicolumn{2}{|l|}{$\mathrm{N}$} & \multicolumn{2}{|l|}{442} & \multicolumn{2}{|l|}{$\mathrm{N}$} & \multicolumn{2}{|l|}{408} & \multicolumn{2}{|l|}{$\mathrm{N}$} & \multicolumn{2}{|l|}{342} \\
\hline & & & \multicolumn{2}{|c|}{$\chi^{2}[\mathrm{df}]$} & \multicolumn{2}{|l|}{.49 n.s. [1] } & \multicolumn{2}{|c|}{$\chi^{2}[\mathrm{df}]$} & \multicolumn{2}{|c|}{.17 n.s. [1] } & $\chi^{2}[\mathrm{~d}$ & & $.00 \mathrm{n}$ & \\
\hline & & & CFI & & 1.00 & & CFI & & 1.00 & & CFI & & 1.00 & \\
\hline Messmo & & & TLI & & 1.01 & & TLI & & 1.01 & & TLI & & 1.01 & \\
\hline & & & RMS & & .00 n.s. & & RMS & & $.00 \mathrm{n}$ & & RMS & & $.00 \mathrm{n}$ & \\
\hline & & & SRM & & .01 & & SRM & & .00 & & SRM & & .00 & \\
\hline
\end{tabular}

$* * \mathrm{p} \leq .01 \quad * \mathrm{p} \leq .05 \quad$ n.s. $=$ nicht signifikant 


\subsubsection{Menschen mit Behinderung als Belastung}

Kurzbezeichnung Variablenname

Instruktion

Quelle

Weiterführende Literatur

Skalierung (Antwortformat)

Inverse Items (-)

Anzahl Items

Skalenbildung

Instrument xa_eb_bl/xb_eb_bl/xc_eb_bl

(xa_ebi_Bel/xb_ebi_Bel/xc_ebi_Bel)

Es folgt nun eine Reihe von verschiedenen Meinungen und möglichen Reaktionen zu Menschen mit Behinderung. Bitte gibt zu jeder Aussage an, inwieweit du ihr zustimmst.

Eigenentwicklung basierend auf Seifert \& Stangl, 1981; Yuker, Block \& Younng, 1970

Cloerkes, 2007; Kastl, 2017; Watson et al., 2014

Endpolbeschriftung; 1 = stimme überhaupt nicht zu, $(\ldots), 6=$ stimme voll $\mathrm{zu}$

6

per Mittelwert

Hauptfragebogen Messzeitpunkt 1, 2 und 3

\begin{tabular}{|c|c|c|c|}
\hline \multicolumn{4}{|c|}{ Itembezeichnung } \\
\hline MZP1 & MZP2 & MZP3 & Itemwortlaut \\
\hline a_eb22 & b_eb22 & c_eb22 & Die meisten Menschen mit Behinderung fühlen sich minderwertig. \\
\hline a_eb25 & b_eb25 & c_eb25 & Von einem Menschen mit Behinderung sollte man nicht zu viel erwarten. \\
\hline a_eb26 & b_eb26 & c_eb26 & $\begin{array}{l}\text { Die Zusammenarbeit mit einem Menschen mit Behinderung bringt für alle } \\
\text { anderen viele Belastungen mit sich. }\end{array}$ \\
\hline a_eb29 & b_eb29 & c_eb29 & $\begin{array}{l}\text { Menschen mit Behinderung sind fast immer auf die Hilfe anderer Men- } \\
\text { schen angewiesen. }\end{array}$ \\
\hline a_eb30 & b_eb30 & c_eb30 & $\begin{array}{l}\text { Menschen mit Behinderung erwarten oft, dass man auf sie besondere Rück- } \\
\text { sicht nimmt. }\end{array}$ \\
\hline a_eb32 & b_eb32 & c_eb32 & Menschen mit Behinderung fallen ihren Mitmenschen oft zur Last. \\
\hline
\end{tabular}




\begin{tabular}{|c|c|c|c|c|c|c|c|c|c|c|c|c|c|c|}
\hline \multirow{2}{*}{\multicolumn{3}{|c|}{ Itembezeichnung }} & \multicolumn{12}{|c|}{ Itemkennwerte } \\
\hline & & & \multicolumn{4}{|c|}{ MZP1 } & \multicolumn{4}{|c|}{ MZP2 } & \multicolumn{4}{|c|}{ MZP3 } \\
\hline MZP1 & MZP2 & MZP3 & MW & SD & $r_{i t}$ & $\lambda_{\mathrm{ij}}$ & MW & $\mathrm{SD}$ & $r_{i t}$ & $\lambda_{\mathrm{ij}}$ & MW & $\mathrm{SD}$ & $r_{i t}$ & $\lambda_{\mathrm{ij}}$ \\
\hline a_eb22 & b_eb22 & c_eb22 & 2.32 & 1.16 & .38 & $.44^{* *}$ & 2.28 & 1.10 & .45 & $.50 * *$ & 2.23 & 1.18 & .41 & $.46^{* *}$ \\
\hline a_eb25 & b_eb25 & c_eb25 & 1.97 & 1.23 & .33 & $.44 * *$ & 2.04 & 1.12 & .41 & $.50 * *$ & 1.99 & 1.03 & .47 & $.53 * *$ \\
\hline a_eb26 & b_eb26 & c_eb26 & 2.92 & 1.18 & .40 & $.62^{* *}$ & 2.69 & 1.17 & .51 & $.68^{* *}$ & 2.69 & 1.11 & .49 & $.67 * *$ \\
\hline a_eb29 & b_eb29 & c_eb29 & 3.18 & 1.24 & .37 & $.47 * *$ & 3.20 & 1.21 & .44 & $.51 * *$ & 3.13 & 1.14 & .43 & $.50 * *$ \\
\hline a_eb30 & b_eb30 & c_eb30 & 2.32 & 1.19 & .40 & $.58 * *$ & 2.29 & 1.11 & .43 & $.57^{* *}$ & 2.42 & 1.19 & .44 & $.60 * *$ \\
\hline a_eb32 & b_eb32 & c_eb32 & 2.57 & 1.11 & .48 & $.58^{* *}$ & 2.55 & 1.05 & .52 & $.61 * *$ & 2.60 & 1.15 & .57 & $.70 * *$ \\
\hline \multirow{4}{*}{\multicolumn{3}{|c|}{ Skalenkennwerte }} & \multicolumn{2}{|c|}{ Cronbachs $\alpha$} & \multicolumn{2}{|l|}{.66} & \multicolumn{2}{|c|}{ Cronbachs $\alpha$} & \multicolumn{2}{|l|}{.75} & \multicolumn{2}{|c|}{ Cronbachs $\alpha$} & \multicolumn{2}{|l|}{.74} \\
\hline & & & \multicolumn{2}{|l|}{ MW } & \multicolumn{2}{|l|}{2.56} & \multicolumn{2}{|l|}{ MW } & \multicolumn{2}{|l|}{2.52} & \multicolumn{2}{|l|}{ MW } & \multicolumn{2}{|c|}{2.51} \\
\hline & & & \multicolumn{2}{|l|}{$\mathrm{SD}$} & \multicolumn{2}{|l|}{.76} & \multicolumn{2}{|l|}{ SD } & \multicolumn{2}{|l|}{.76} & \multicolumn{2}{|l|}{ SD } & \multicolumn{2}{|l|}{.76} \\
\hline & & & \multicolumn{2}{|l|}{$\mathrm{N}$} & \multicolumn{2}{|l|}{441} & \multicolumn{2}{|l|}{$\mathrm{N}$} & \multicolumn{2}{|l|}{411} & \multicolumn{2}{|l|}{$\mathrm{N}$} & \multicolumn{2}{|l|}{343} \\
\hline & & & \multicolumn{2}{|c|}{$\chi^{2}[\mathrm{df}]$} & \multicolumn{2}{|c|}{14.51 n.s. [8] } & \multicolumn{2}{|c|}{$\chi^{2}[\mathrm{df}]$} & 21.5 & & $\chi^{2}[\mathrm{~d} f$ & & 20.2 & \\
\hline & & & CFI & & .98 & & CFI & & .97 & & CFI & & .97 & \\
\hline Messmo & & & TLI & & .96 & & TLI & & .93 & & TLI & & .94 & \\
\hline & & & RMS & & $.04 \mathrm{r}$ & & RMS & & $.06 \mathrm{n}$ & & RMS & & .07 & \\
\hline & & & SRM & & .03 & & SRM & & .03 & & SRM & & .03 & \\
\hline
\end{tabular}

** $\mathrm{p} \leq .01 \quad * \mathrm{p} \leq .05 \quad$ n.s. $=$ nicht signifikant 


\subsubsection{Menschen mit Behinderung als gleich}

Kurzbezeichnung Variablenname

Instruktion

Quelle

Weiterführende Literatur

Skalierung (Antwortformat)

Inverse Items (-)

Anzahl Items

Skalenbildung

Instrument xa_eb_gl/xb_eb_gl/xc_eb_gl

(xa_ebi_In/xb_ebi_In/xc_ebi_In)

Es folgt nun eine Reihe von verschiedenen Meinungen und möglichen Reaktionen zu Menschen mit Behinderung. Bitte gibt zu jeder Aussage an, inwieweit du ihr zustimmst.

Eigenentwicklung basierend auf Seifert \& Stangl, 1981

Cloerkes, 2007; Kastl, 2017; Watson et al., 2014

Endpolbeschriftung; 1 = stimme überhaupt nicht zu, $(\ldots), 6=$ stimme voll $\mathrm{zu}$

6

per Mittelwert

Hauptfragebogen Messzeitpunkt 1, 2 und 3

\begin{tabular}{|c|c|c|c|}
\hline \multicolumn{4}{|c|}{ Itembezeichnung } \\
\hline MZP1 & MZP2 & MZP3 & Itemwortlaut \\
\hline a_eb28 & b_eb28 & c_eb28 & $\begin{array}{l}\text { Mit einem Menschen mit Behinderung kann man genauso gut die Freizeit } \\
\text { verbringen wie mit einem Menschen ohne Behinderung. }\end{array}$ \\
\hline a_eb31 & b_eb31 & c_eb31 & $\begin{array}{l}\text { Menschen mit Behinderung sind beruflich genauso leistungsfähig wie Men- } \\
\text { schen ohne Behinderung. }\end{array}$ \\
\hline a_eb33 & b_eb33 & c_eb33 & $\begin{array}{l}\text { Mit Menschen mit Behinderung kommt man im Allgemeinen genauso gut } \\
\text { zurecht wie mit Menschen ohne Behinderung. }\end{array}$ \\
\hline a_eb45 & b_eb45 & c_eb45 & Menschen mit und ohne Behinderung haben einen ähnlichen Alltag. \\
\hline a_eb51 & b_eb51 & c_eb51 & $\begin{array}{l}\text { Menschen mit Behinderung haben die gleichen Probleme wie Menschen } \\
\text { ohne Behinderung. }\end{array}$ \\
\hline a_eb56 & b_eb55 & c_eb55 & $\begin{array}{l}\text { Menschen mit Behinderung haben die gleichen Bedürfnisse wie Menschen } \\
\text { ohne Behinderung. }\end{array}$ \\
\hline
\end{tabular}




\begin{tabular}{|c|c|c|c|c|c|c|c|c|c|c|c|c|c|c|}
\hline \multirow{2}{*}{\multicolumn{3}{|c|}{ Itembezeichnung }} & \multicolumn{12}{|c|}{ Itemkennwerte } \\
\hline & & & \multicolumn{4}{|c|}{ MZP1 } & \multicolumn{4}{|c|}{ MZP2 } & \multicolumn{4}{|c|}{ MZP3 } \\
\hline MZP1 & MZP2 & MZP3 & MW & SD & $\mathrm{r}_{\mathrm{it}}$ & $\lambda_{\mathrm{ij}}$ & MW & SD & $r_{i t}$ & $\lambda_{\mathrm{ij}}$ & MW & SD & $\mathrm{r}_{\mathrm{it}}$ & $\lambda_{\mathrm{ij}}$ \\
\hline a_eb28 & b_eb28 & c_eb28 & 4.91 & 1.23 & .51 & $.64^{* *}$ & 4.81 & 1.25 & .50 & $.63^{* *}$ & 4.79 & 1.23 & .51 & $.63 * *$ \\
\hline a_eb31 & b_eb31 & c_eb31 & 3.44 & 1.40 & .48 & $.62 * *$ & 3.55 & 1.37 & .43 & $.55^{* *}$ & 3.52 & 1.36 & .48 & $.57^{* *}$ \\
\hline a_eb33 & b_eb33 & c_eb33 & 4.61 & 1.32 & .48 & $.60^{* *}$ & 4.64 & 1.27 & .51 & $.66^{* *}$ & 4.69 & 1.23 & .51 & $.63 * *$ \\
\hline a_eb45 & b_eb45 & c_eb45 & 3.61 & 1.33 & .40 & $.46^{* *}$ & 3.82 & 1.35 & .37 & $.43^{* *}$ & 3.94 & 1.40 & .46 & $.53 * *$ \\
\hline a_eb51 & b_eb51 & c_eb51 & 3.78 & 1.39 & .45 & $.50^{* *}$ & 3.88 & 1.34 & .44 & $.50^{* *}$ & 3.95 & 1.41 & .47 & $.53 * *$ \\
\hline a_eb56 & b_eb55 & c_eb55 & 5.21 & 1.08 & .36 & $.39 * *$ & 5.16 & 1.06 & .39 & $.45^{* *}$ & 5.08 & 1.19 & .45 & $.54 * *$ \\
\hline \multirow{4}{*}{\multicolumn{3}{|c|}{ Skalenkennwerte }} & \multicolumn{2}{|c|}{ Cronbachs $\alpha$} & \multicolumn{2}{|l|}{.66} & \multicolumn{2}{|c|}{ Cronbachs $\alpha$} & \multicolumn{2}{|l|}{.71} & \multicolumn{2}{|c|}{ Cronbachs $\alpha$} & \multicolumn{2}{|l|}{.75} \\
\hline & & & \multicolumn{2}{|l|}{ MW } & \multicolumn{2}{|l|}{4.27} & \multicolumn{2}{|l|}{ MW } & \multicolumn{2}{|c|}{4.29} & \multicolumn{2}{|l|}{ MW } & \multicolumn{2}{|l|}{4.32} \\
\hline & & & \multicolumn{2}{|l|}{ SD } & \multicolumn{2}{|l|}{.83} & \multicolumn{2}{|l|}{ SD } & \multicolumn{2}{|l|}{.83} & \multicolumn{2}{|l|}{ SD } & \multicolumn{2}{|l|}{.87} \\
\hline & & & \multicolumn{2}{|l|}{$\mathrm{N}$} & \multicolumn{2}{|l|}{442} & \multicolumn{2}{|l|}{$\mathrm{N}$} & \multicolumn{2}{|l|}{409} & \multicolumn{2}{|l|}{$\mathrm{N}$} & \multicolumn{2}{|l|}{342} \\
\hline & & & \multicolumn{2}{|c|}{$\chi^{2}[\mathrm{df}]$} & \multicolumn{2}{|l|}{$44.58^{* *}[9]$} & \multicolumn{2}{|c|}{$\chi^{2}[\mathrm{df}]$} & 38.6 & & $\chi^{2}[\mathrm{~d}$ & & 42.0 & \\
\hline & & & CFI & & .91 & & CFI & & .92 & & CFI & & .91 & \\
\hline Messmo & & & TLI & & .85 & & TLI & & .87 & & TLI & & .85 & \\
\hline & & & RMS & & $.09 * *$ & & RMSI & & $.09 *$ & & RMS & & $.10^{* \ngtr}$ & \\
\hline & & & SRM & & .05 & & SRMl & & .05 & & SRM & & .05 & \\
\hline
\end{tabular}

${ }^{*} \mathrm{p} \leq .01 \quad * \mathrm{p} \leq .05 \quad$ n.s. $=$ nicht signifikant 


\subsubsection{Menschen mit Behinderung als anders}

Kurzbezeichnung Variablenname

Instruktion

Quelle

Weiterführende Literatur

Skalierung (Antwortformat)

Inverse Items (-)

Anzahl Items

Skalenbildung

Instrument xa_eb_an/xb_eb_an/xc_eb_an

(xa_ebi_Out/xb_ebi_Out/xc_ebi_Out)

Es folgt nun eine Reihe von verschiedenen Meinungen und möglichen Reaktionen zu Menschen mit Behinderung. Bitte gibt zu jeder Aussage an, inwieweit du ihr zustimmst.

Eigenentwicklung basierend auf Seifert \& Stangl, 1981; Kuhl, Moser, Schäfer \& Redlich, 2013

Cloerkes, 2007; Kastl, 2017; Watson et al., 2014

Endpolbeschriftung; 1 = stimme überhaupt nicht zu, $(\ldots), 6=$ stimme voll $\mathrm{zu}$

7

per Mittelwert

Hauptfragebogen Messzeitpunkt 1, 2 und 3

\begin{tabular}{|c|c|c|c|}
\hline \multicolumn{4}{|c|}{ Itembezeichnung } \\
\hline MZP1 & MZP2 & MZP3 & Itemwortlaut \\
\hline a_eb21 & b_eb21 & c_eb21 & Menschen mit Behinderung bleiben am liebsten unter sich. \\
\hline a_eb23 & b_eb23 & c_eb23 & $\begin{array}{l}\text { Es ist schwierig, sich gegenüber einem Menschen mit Behinderung richtig } \\
\text { zu verhalten. }\end{array}$ \\
\hline a_eb27 & b_eb27 & c_eb27 & $\begin{array}{l}\text { Menschen mit Behinderung können meistens nur einfache berufliche Tä- } \\
\text { tigkeiten ausüben. }\end{array}$ \\
\hline a_eb41 & b_eb41 & c_eb41 & $\begin{array}{l}\text { Menschen, die dieselbe Behinderung haben, sind sich meistens recht ähn- } \\
\text { lich. }\end{array}$ \\
\hline a_eb46 & b_eb46 & c_eb46 & $\begin{array}{l}\text { Ohne das entsprechende Fachwissen kann man Menschen mit Behinderung } \\
\text { nicht wirklich verstehen. }\end{array}$ \\
\hline a_eb49 & b_eb49 & c_eb49 & $\begin{array}{l}\text { Menschen mit Behinderung kommen besser mit anderen Menschen mit } \\
\text { Behinderung klar als mit Menschen ohne Behinderung. }\end{array}$ \\
\hline a_eb53 & b_eb53 & c_eb53 & $\begin{array}{l}\text { Menschen ohne Behinderung haben nicht viel gemeinsam mit Menschen } \\
\text { mit Behinderung. }\end{array}$ \\
\hline
\end{tabular}




\begin{tabular}{|c|c|c|c|c|c|c|c|c|c|c|c|c|c|c|}
\hline \multirow{2}{*}{\multicolumn{3}{|c|}{ Itembezeichnung }} & \multicolumn{12}{|c|}{ Itemkennwerte } \\
\hline & & & \multicolumn{4}{|c|}{ MZP1 } & \multicolumn{4}{|c|}{ MZP2 } & \multicolumn{4}{|c|}{ MZP3 } \\
\hline MZP1 & MZP2 & MZP3 & MW & SD & $\mathrm{r}_{\mathrm{it}}$ & $\lambda_{\mathrm{ij}}$ & MW & SD & $\mathrm{r}_{\mathrm{it}}$ & $\lambda_{\mathrm{ij}}$ & MW & SD & $r_{i t}$ & $\lambda_{\mathrm{ij}}$ \\
\hline a_eb21 & b_eb21 & c_eb21 & 1.75 & .98 & .44 & $.57 * *$ & 1.92 & 1.00 & .34 & $.42 * *$ & 1.90 & 1.11 & .53 & $.67 * *$ \\
\hline a_eb23 & b_eb23 & c_eb23 & 2.65 & 1.28 & .37 & $.47 * *$ & 2.48 & 1.27 & .35 & $.44 * *$ & 2.25 & 1.17 & .50 & $.59 * *$ \\
\hline a_eb27 & b_eb27 & c_eb27 & 3.04 & 1.28 & .31 & $.41 * *$ & 2.86 & 1.27 & .39 & $.49 * *$ & 2.79 & 1.27 & .34 & $.40^{* *}$ \\
\hline a_eb41 & b_eb41 & c_eb41 & 2.57 & 1.32 & .37 & $.47 * *$ & 2.64 & 1.34 & .37 & $.43^{* *}$ & 2.67 & 1.37 & .33 & $.39 * *$ \\
\hline a_eb46 & b_eb46 & c_eb46 & 2.39 & 1.22 & .41 & $.53^{* *}$ & 2.44 & 1.20 & .44 & $.54 * *$ & 2.34 & 1.18 & .42 & $.52 * *$ \\
\hline a_eb49 & b_eb49 & c_eb49 & 2.79 & 1.29 & .39 & $.47^{* *}$ & 2.77 & 1.22 & .39 & $.50 * *$ & 2.82 & 1.30 & .47 & $.57 * *$ \\
\hline a_eb53 & b_eb53 & c_eb53 & 1.95 & 1.05 & .46 & $.57 * *$ & 2.04 & 1.02 & .55 & $.71^{* *}$ & 2.16 & 1.18 & .52 & $.59 * *$ \\
\hline \multirow{4}{*}{\multicolumn{3}{|c|}{ Skalenkennwerte }} & \multicolumn{2}{|c|}{ Cronbachs $\alpha$} & \multicolumn{2}{|l|}{.77} & \multicolumn{2}{|c|}{ Cronbachs $\alpha$} & \multicolumn{2}{|l|}{.70} & \multicolumn{2}{|c|}{ Cronbachs $\alpha$} & \multicolumn{2}{|l|}{.73} \\
\hline & & & \multicolumn{2}{|l|}{ MW } & \multicolumn{2}{|l|}{2.46} & \multicolumn{2}{|l|}{ MW } & \multicolumn{2}{|c|}{2.45} & \multicolumn{2}{|l|}{ MW } & \multicolumn{2}{|l|}{2.43} \\
\hline & & & \multicolumn{2}{|l|}{ SD } & \multicolumn{2}{|l|}{.73} & \multicolumn{2}{|l|}{ SD } & \multicolumn{2}{|l|}{.73} & \multicolumn{2}{|l|}{ SD } & \multicolumn{2}{|l|}{.77} \\
\hline & & & \multicolumn{2}{|l|}{$\mathrm{N}$} & \multicolumn{2}{|l|}{442} & \multicolumn{2}{|l|}{$\mathrm{N}$} & \multicolumn{2}{|l|}{411} & \multicolumn{2}{|l|}{$\mathrm{N}$} & \multicolumn{2}{|l|}{343} \\
\hline & & & \multicolumn{2}{|c|}{$\chi^{2}[\mathrm{df}]$} & \multicolumn{2}{|c|}{$29.19^{* *}[14]$} & $\chi^{2}[\mathrm{df}$ & & 19.2 & [14] & $\chi^{2}[\mathrm{~d} f$ & & 24.12 & \\
\hline & & & CFI & & .96 & & CFI & & .99 & & CFI & & .97 & \\
\hline Messmo & & & TLI & & .94 & & TLI & & .98 & & TLI & & .96 & \\
\hline & & & RMS & & $.05 \mathrm{n}$ & & RMSI & & $.03 \mathrm{n}$ & & RMS & & $.05 \mathrm{n}$ & \\
\hline & & & SRM & & .03 & & SRMl & & .03 & & SRM & & .03 & \\
\hline
\end{tabular}

** $\mathrm{p} \leq .01 \quad * \mathrm{p} \leq .05 \quad$ n.s. $=$ nicht signifikant 


\subsubsection{Unsicherheit im Kontakt mit Menschen mit Behinderung}

Kurzbezeichnung Variablenname

Instruktion

Quelle

Weiterführende Literatur

Skalierung (Antwortformat)

Inverse Items (-)

Anzahl Items

Skalenbildung

Instrument xa_eb_un/xb_eb_un/xc_eb_un (xa_ebi_UiK/xb_ebi_UiK/xc_ebi_UiK)

Es folgt nun eine Reihe von verschiedenen Meinungen und möglichen Reaktionen zu Menschen mit Behinderung. Bitte gibt zu jeder Aussage an, inwieweit du ihr zustimmst.

Adaptiert von Gething \& Wheeler, 1992; Seifert \& Stangl, 1981

Cloerkes, 2007; Kastl, 2017; Watson et al., 2014

Endpolbeschriftung; 1 = stimme überhaupt nicht $\mathrm{zu}$, $(\ldots), 6=$ stimme voll $\mathrm{zu}$

6

per Mittelwert

Hauptfragebogen Messzeitpunkt 1, 2 und 3

\begin{tabular}{|c|c|c|c|}
\hline MZP1 & MZP2 & MZP3 & Itemwortlaut \\
\hline a_eb9 & b_eb9 & c_eb9 & Ich fühle mich unwohl und es fällt mir schwer, entspannt zu sein. \\
\hline a_eb11 & b_eb11 & c_eb11 & $\begin{array}{l}\text { Wenn ich Menschen mit Behinderung sehe, kann ich nicht anders, als im- } \\
\text { mer wieder hinzuschauen. }\end{array}$ \\
\hline a_eb12 & b_eb12 & c_eb12 & $\begin{array}{l}\text { Beim Kontakt mit Menschen mit Behinderung fühle ich mich unsicher, weil } \\
\text { ich nicht weiß, wie ich mich verhalten soll. }\end{array}$ \\
\hline a_eb17 & b_eb17 & c_eb17 & $\begin{array}{l}\text { Ich habe Angst, Menschen mit Behinderung direkt in die Augen zu schau- } \\
\text { en. }\end{array}$ \\
\hline a_eb18 & b_eb18 & c_eb18 & $\begin{array}{l}\text { Ich halte Kontakte zu Menschen mit Behinderung meist kurz und beende } \\
\text { sie so schnell wie möglich. }\end{array}$ \\
\hline a_eb24 & b_eb24 & c_eb24 & $\begin{array}{l}\text { Wenn ich einem Menschen mit Behinderung begegne, habe ich immer } \\
\text { irgendwie ein schlechtes Gewissen. }\end{array}$ \\
\hline
\end{tabular}




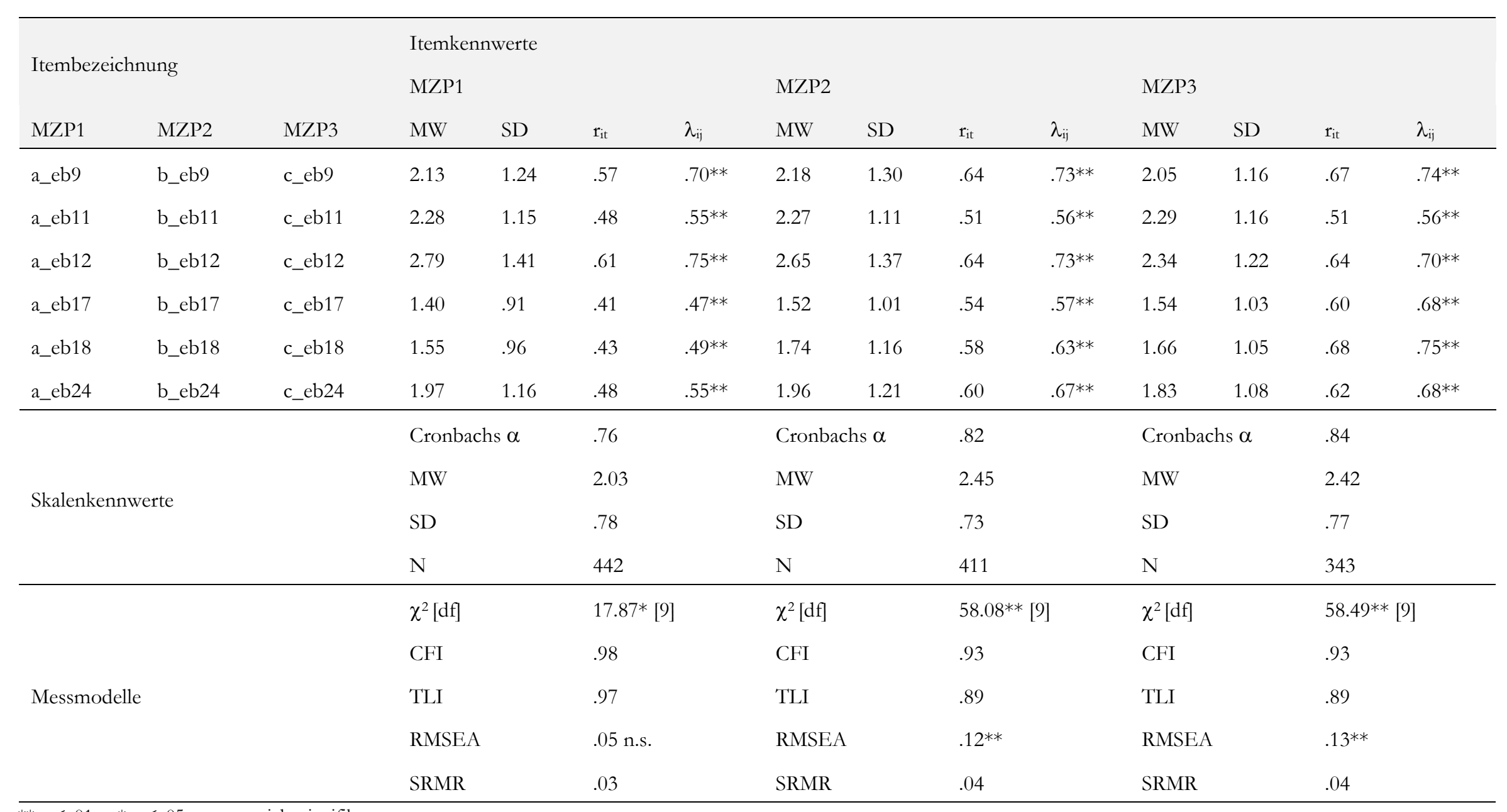

** $\mathrm{p} \leq .01 \quad * \mathrm{p} \leq .05 \quad$ n.s. $=$ nicht signifikant 


\subsubsection{Behinderung als Bedrohung}

Kurzbezeichnung Variablenname

Instruktion

Quelle

Weiterführende Literatur

Skalierung (Antwortformat)

Inverse Items (-)

Anzahl Items

Skalenbildung

Instrument xa_eb_dr/xb_eb_dr/xc_eb_dr

(xa_ebi_BeaBel/xb_ebi_BeaBel/xc_ebi_BeaBel)

Es folgt nun eine Reihe von verschiedenen Meinungen und möglichen Reaktionen zu Menschen mit Behinderung. Bitte gibt zu jeder Aussage an, inwieweit du ihr zustimmst.

Eigenentwicklung angelehnt an Gething \& Wheeler, 1992

Cloerkes, 2007; Kastl, 2017; Watson et al., 2014

Endpolbeschriftung; 1 = stimme überhaupt nicht zu, $(\ldots), 6=$ stimme voll $\mathrm{zu}$

4

per Mittelwert

Hauptfragebogen Messzeitpunkt 1, 2 und 3

\begin{tabular}{llll}
\hline \multicolumn{2}{l}{ Itembezeichnung } & & \\
MZP1 & MZP2 & MZP3 & Itemwortlaut \\
\hline a_eb7 & b_eb7 & c_eb7 & Ich bin dankbar, selbst keine solche Belastung zu haben. \\
a_eb34 & b_eb34 & c_eb34 & Für mich wäre es schrecklich, selbst eine Behinderung zu haben. \\
a_eb36 & b_eb36 & c_eb36 & $\begin{array}{l}\text { Wenn ich eine starke Behinderung hätte, fände ich mein Leben nicht le- } \\
\text { benswert. }\end{array}$ \\
a_eb39 & b_eb39 & c_eb39 & Ein Kind mit Behinderung zu bekommen, wäre für mich ein Albtraum. \\
\hline
\end{tabular}




\begin{tabular}{|c|c|c|c|c|c|c|c|c|c|c|c|c|c|c|}
\hline \multirow{2}{*}{\multicolumn{3}{|c|}{ Itembezeichnung }} & \multicolumn{12}{|c|}{ Itemkennwerte } \\
\hline & & & \multicolumn{4}{|c|}{ MZP1 } & \multicolumn{4}{|c|}{ MZP2 } & \multicolumn{4}{|c|}{ MZP3 } \\
\hline MZP1 & MZP2 & MZP3 & MW & $\mathrm{SD}$ & $\mathrm{r}_{\mathrm{it}}$ & $\lambda_{\mathrm{ij}}$ & MW & $\mathrm{SD}$ & $r_{i t}$ & $\lambda_{\mathrm{ij}}$ & MW & $\mathrm{SD}$ & $\mathrm{r}_{\mathrm{it}}$ & $\lambda_{\mathrm{ij}}$ \\
\hline a_eb7 & b_eb7 & c_eb7 & 5.47 & 1.10 & .39 & $.30^{* *}$ & 5.36 & 1.17 & .41 & $.33^{* *}$ & 5.12 & 1.25 & .31 & $.20 * *$ \\
\hline a_eb34 & b_eb34 & c_eb34 & 4.83 & 1.39 & .61 & $.60^{* *}$ & 4.79 & 1.46 & .61 & $.64 * *$ & 4.55 & 1.52 & .52 & $.52 * *$ \\
\hline a_eb36 & b_eb36 & c_eb36 & 2.85 & 1.56 & .43 & $.59 * *$ & 2.81 & 1.47 & .44 & $.59^{* *}$ & 2.93 & 1.52 & .39 & $.53 * *$ \\
\hline a_eb39 & b_eb39 & c_eb39 & 3.37 & 1.64 & .53 & $.75^{* *}$ & 3.27 & 1.57 & .49 & $.70^{* *}$ & 3.45 & 1.68 & .51 & $.84 * *$ \\
\hline \multirow{4}{*}{\multicolumn{3}{|c|}{ Skalenkennwerte }} & \multicolumn{2}{|c|}{ Cronbachs $\alpha$} & \multicolumn{2}{|l|}{.71} & \multicolumn{2}{|c|}{ Cronbachs $\alpha$} & \multicolumn{2}{|l|}{.70} & \multicolumn{2}{|c|}{ Cronbachs $\alpha$} & \multicolumn{2}{|l|}{.62} \\
\hline & & & \multicolumn{2}{|c|}{ MW } & \multicolumn{2}{|l|}{4.16} & \multicolumn{2}{|l|}{ MW } & \multicolumn{2}{|l|}{4.08} & \multicolumn{2}{|l|}{ MW } & \multicolumn{2}{|c|}{4.04} \\
\hline & & & \multicolumn{2}{|l|}{$\mathrm{SD}$} & \multicolumn{2}{|l|}{1.05} & \multicolumn{2}{|l|}{ SD } & \multicolumn{2}{|l|}{1.04} & \multicolumn{2}{|l|}{$\mathrm{SD}$} & \multicolumn{2}{|l|}{1.06} \\
\hline & & & \multicolumn{2}{|l|}{$\mathrm{N}$} & \multicolumn{2}{|l|}{441} & \multicolumn{2}{|l|}{$\mathrm{N}$} & \multicolumn{2}{|l|}{412} & \multicolumn{2}{|l|}{$\mathrm{N}$} & \multicolumn{2}{|l|}{342} \\
\hline & & & \multicolumn{2}{|c|}{$\chi^{2}[\mathrm{df}]$} & \multicolumn{2}{|c|}{1.06 n.s. [1] } & \multicolumn{2}{|c|}{$\chi^{2}[\mathrm{df}]$} & \multicolumn{2}{|l|}{.09 n.s. [1] } & $\chi^{2}[\mathrm{df}$ & & $.00 \mathrm{n}$ & \\
\hline & & & CFI & & 1.00 & & CFI & & 1.00 & & CFI & & 1.00 & \\
\hline Messmo & & & TLI & & 1.00 & & TLI & & 1.02 & & TLI & & 1.03 & \\
\hline & & & RMS & & .01 n.s. & & RMSI & & .00 n.s. & & RMSI & & $.00 \mathrm{n}$ & \\
\hline & & & SRM & & .01 & & SRMl & & .00 & & SRMI & & .00 & \\
\hline
\end{tabular}

** $\mathrm{p} \leq .01 \quad * \mathrm{p} \leq .05 \quad$ n.s. $=$ nicht signifikant 


\subsubsection{Behinderung als Konstruktion}

Kurzbezeichnung Variablenname

Instruktion

Quelle

Weiterführende Literatur

Skalierung (Antwortformat)

Inverse Items (-)

Anzahl Items

Skalenbildung

Instrument xa_eb_no/xb_eb_no/xc_eb_no

(xa_ebi_BeaNor/xb_ebi_BeaNor/xc_ebi_BeaNor)

Es folgt nun eine Reihe von verschiedenen Meinungen und möglichen Reaktionen zu Menschen mit Behinderung. Bitte gibt zu jeder Aussage an, inwieweit du ihr zustimmst.

Eigenentwicklung

Cloerkes, 2007; Kastl, 2017; Watson et al., 2014

Endpolbeschriftung; 1 = stimme überhaupt nicht zu, $(\ldots), 6=$ stimme voll $\mathrm{zu}$

6

per Mittelwert

Hauptfragebogen Messzeitpunkt 1, 2 und 3

Itembezeichnung

MZP1 MZP2 MZP3 Itemwortlaut

\begin{tabular}{|c|c|c|c|}
\hline a_eb38 & b_eb38 & c_eb38 & $\begin{array}{l}\text { Menschen mit Behinderung sind genauso unterschiedlich wie Menschen } \\
\text { ohne Behinderung. }\end{array}$ \\
\hline a_eb40 & b_eb40 & c_eb40 & $\begin{array}{l}\text { Alle Menschen können auf unterschiedliche Weise durch etwas behindert } \\
\text { sein oder behindert werden. }\end{array}$ \\
\hline a_eb44 & b_eb44 & c_eb44 & Es ist nicht außergewöhnlich, eine Behinderung zu haben. \\
\hline a_eb48 & b_eb48 & c_eb48 & $\begin{array}{l}\text { Wenn die gesellschaftlichen Rahmenbedingungen stimmen würden, könn- } \\
\text { ten Menschen mit Behinderung ohne Einschränkungen leben. }\end{array}$ \\
\hline a_eb50 & b_eb50 & c_eb50 & Was eine Behinderung ist, lässt sich nicht ein für alle Mal definieren. \\
\hline a_eb54 & b_eb54 & c_eb54 & Was als Behinderung gilt, hängt immer vom gesellschaftlichen Kontext ab. \\
\hline
\end{tabular}




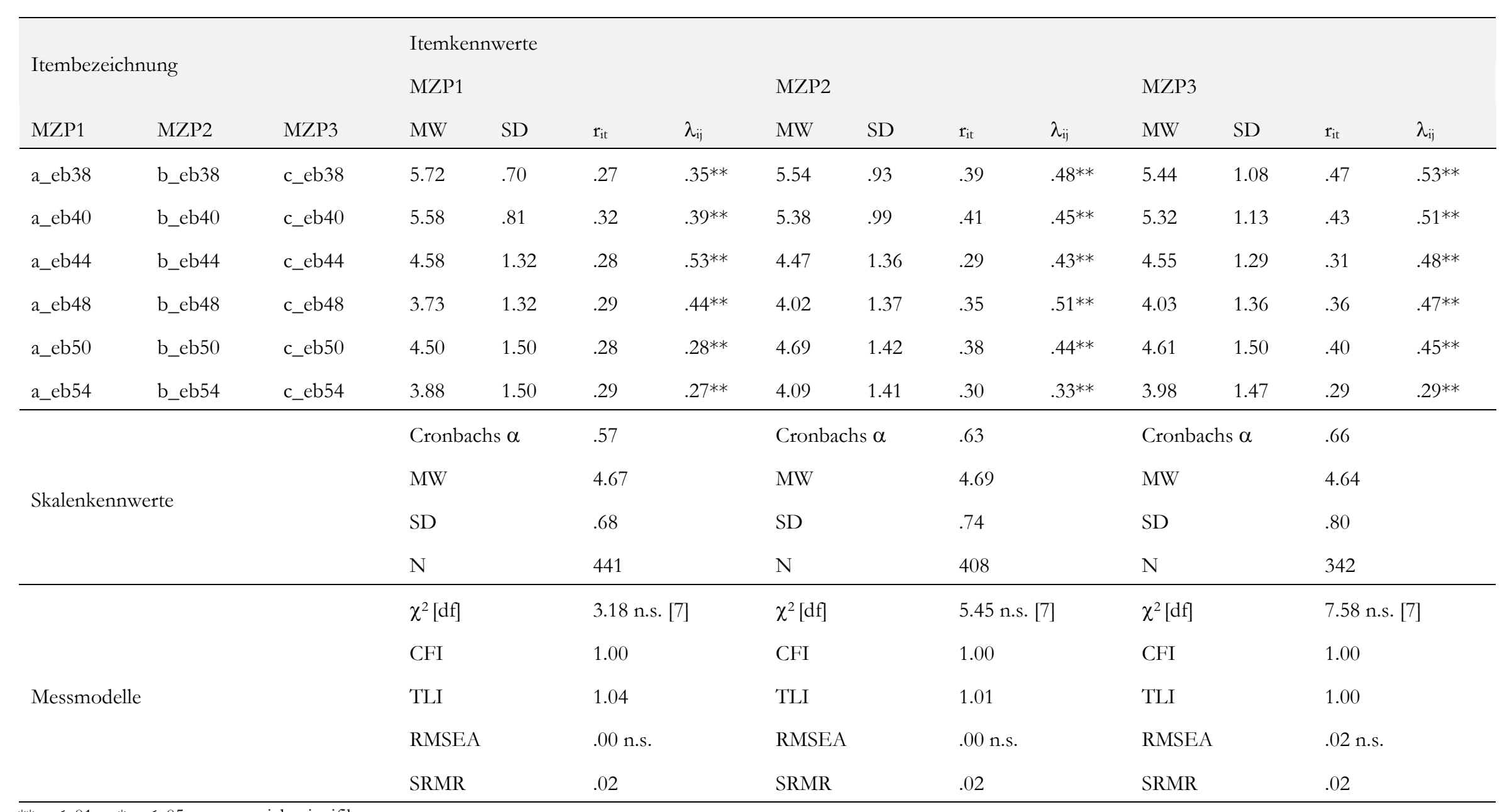

** $\mathrm{p} \leq .01 \quad * \mathrm{p} \leq .05 \quad$ n.s. $=$ nicht signifikant 
1.1.8 Strukturmodell Einstellungen zu (Menschen mit) Behinderung

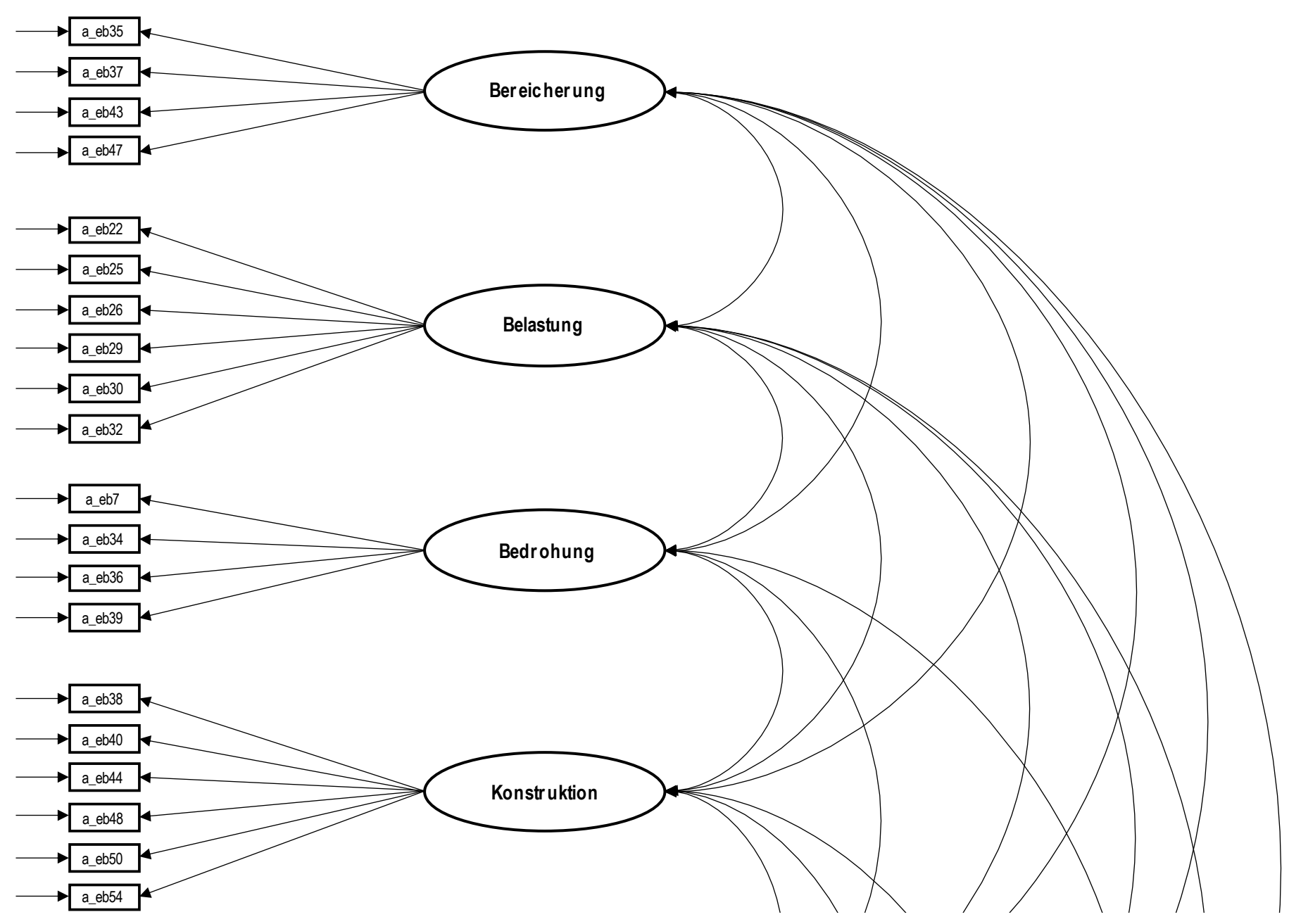




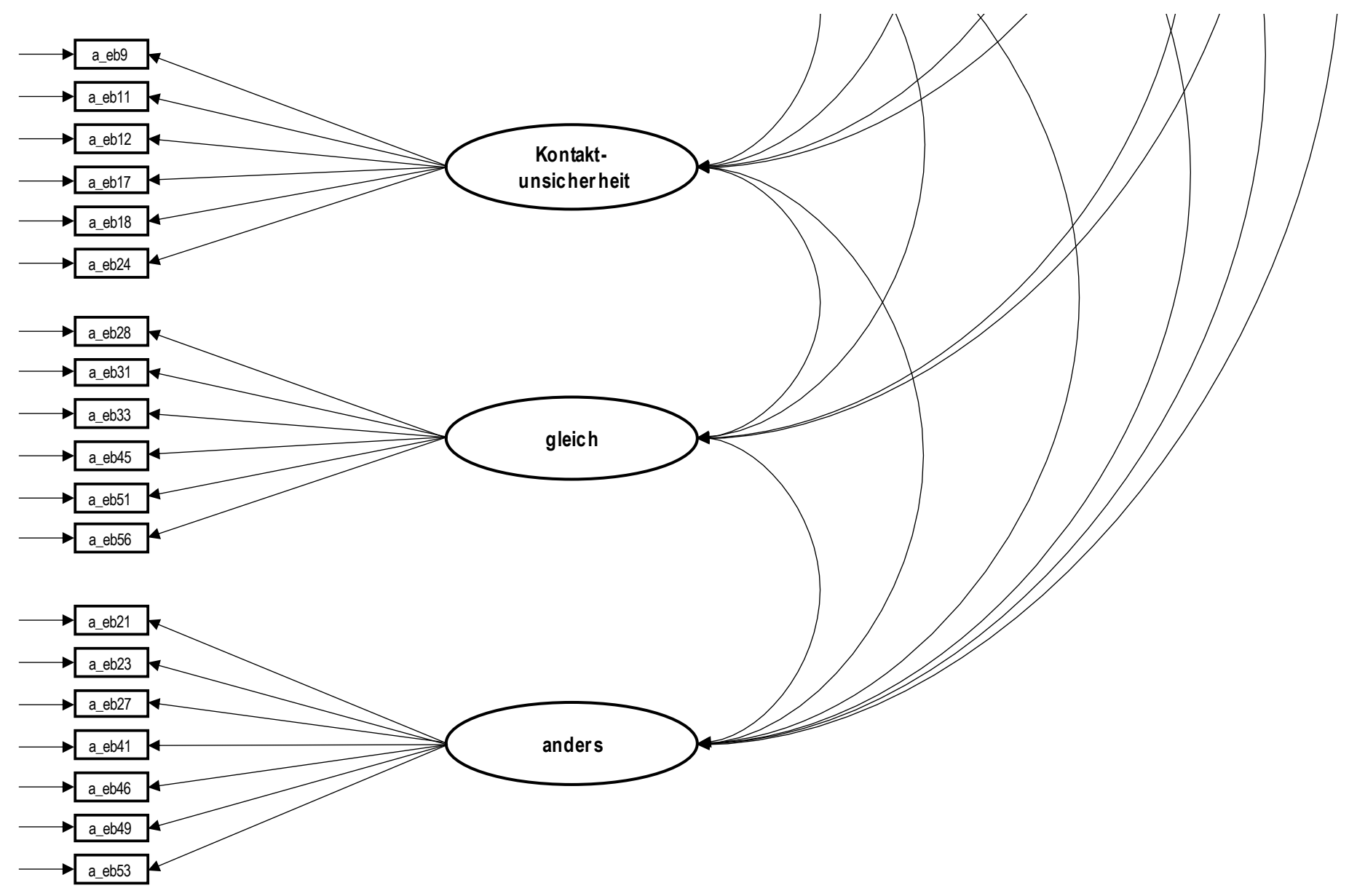

Modellfitwerte (MZP1/MZP2/MZP3):

$\chi^{2}[\mathrm{df}]=1235.85^{* *}[674] / 1345.54 * *[674] / 1360.87^{* *}[674] ; \mathrm{CFI}=.86 / .84 / .84 ; \mathrm{TLI}=.84 / .83 / .83 ; \mathrm{RMSEA}=.04$ n.s. $/ .05$ n.s. $/ .06 * ;$ SRMR $=.06 / .06 / .06$ 


\subsubsection{Interkorrelationen}

\begin{tabular}{lllllllc}
\hline MZP1 & xa_eb_br & xa_eb_bl & xa_eb_gl & xa_eb_an & xa_eb_un & xa_eb_dr & xa_eb_no \\
\hline xa_eb_br & -- & $-.38^{* *}$ & $.61^{* *}$ & $-.50^{* *}$ & $-.31 * *$ & $-.29 * *$ & $.86^{* *}$ \\
xa_eb_bl & $-.38^{* *}$ & -- & $-.57^{* *}$ & $.88^{* *}$ & $.49^{* *}$ & $.52^{* *}$ & $-.50^{* *}$ \\
xa_eb_gl & $.61^{* *}$ & $-.57^{* *}$ & -- & $-.54^{* *}$ & $-.33^{* *}$ & $-.50^{* *}$ & $.82^{* *}$ \\
xa_eb_an & $-.50^{* *}$ & $.88^{* *}$ & $-.54^{* *}$ & -- & $.71^{* *}$ & $.58^{* *}$ & $-.50^{* *}$ \\
xa_eb_un & $-.31^{* *}$ & $.49^{* *}$ & $-.33^{* *}$ & $.71^{* *}$ & -- & $.50^{* *}$ & $-.32^{* *}$ \\
xa_eb_dr & $-.29^{* *}$ & $.52^{* *}$ & $-.50^{* *}$ & $.58^{* *}$ & $.50^{* *}$ & -- & $-.38^{* *}$ \\
xa_eb_no & $.86^{* *}$ & $-.50^{* *}$ & $.82^{* *}$ & $-.50^{* *}$ & $-.32^{* *}$ & $-.38^{* *}$ & -- \\
\hline
\end{tabular}

\begin{tabular}{llllllll}
\hline MZP2 & xa_eb_br & xa_eb_bl & xa_eb_gl & xa_eb_an & xa_eb_un & xa_eb_dr & xa_eb_no \\
\hline xa_eb_br & -- & $-.37^{* *}$ & $.66^{* *}$ & $-.50^{* *}$ & $-.35^{* *}$ & $-.32^{* *}$ & $.87^{* *}$ \\
xa_eb_bl & $-.37 * *$ & -- & $-.56^{* *}$ & $.86^{* *}$ & $.61^{* *}$ & $.56^{* *}$ & $-.42^{* *}$ \\
xa_eb_gl & $.66^{* *}$ & $-.56^{* *}$ & -- & $-.58^{* *}$ & $-.44^{* *}$ & $-.35^{* *}$ & $.84^{* *}$ \\
xa_eb_an & $-.50^{* *}$ & $.86^{* *}$ & $-.58^{* *}$ & -- & $.73^{* *}$ & $.53^{* *}$ & $-.55^{* *}$ \\
xa_eb_un & $-.35^{* *}$ & $.61^{* *}$ & $-.44^{* *}$ & $.73^{* *}$ & -- & $.57^{* *}$ & $-.43^{* *}$ \\
xa_eb_dr & $-.32^{* *}$ & $.56^{* *}$ & $-.35^{* *}$ & $.53^{* *}$ & $.57^{* *}$ & -- & $-.25^{* *}$ \\
xa_eb_no & $.87^{* *}$ & $-.42^{* *}$ & $.84^{* *}$ & $-.55^{* *}$ & $-.43^{* *}$ & $-.25^{* *}$ & -- \\
\hline
\end{tabular}

\begin{tabular}{|c|c|c|c|c|c|c|c|}
\hline MZP3 & xa_eb_br & xa_eb_bl & xa_eb_gl & xa_eb_an & xa_eb_un & xa_eb_dr & xa_eb_no \\
\hline xa_eb_br & -- & $-.46 * *$ & $.77 * *$ & $-.50 * *$ & $-.40 * *$ & $-.31 * *$ & $.86^{* *}$ \\
\hline xa_eb_bl & $-.46 * *$ & -- & $-.61 * *$ & $.84 * *$ & $.58^{* *}$ & $.59 * *$ & $-.63 * *$ \\
\hline xa_eb_gl & $.77 * *$ & $-.61 * *$ & -- & $-.60 * *$ & $-.41 * *$ & $-.41 * *$ & $.95^{* *}$ \\
\hline xa_eb_an & $-.50 * *$ & $.84 * *$ & $-.60 * *$ & -- & $.81^{* *}$ & $.48^{* *}$ & $-.73 * *$ \\
\hline xa_eb_un & $-.40 * *$ & $.58^{* *}$ & $-.41 * *$ & $.81^{* *}$ & -- & $.50^{* *}$ & $-.63 * *$ \\
\hline xa_eb_dr & $-.31 * *$ & $.59 * *$ & $-.41 * *$ & $.48^{* *}$ & $.50^{* *}$ & -- & $-.35^{* *}$ \\
\hline xa_eb_no & $.86^{* *}$ & $-.63 * *$ & $.95^{* *}$ & $-.73 * *$ & $-.63 * *$ & $-.35^{* *}$ & -- \\
\hline
\end{tabular}

${ }^{* *} \mathrm{p} \leq .01 \quad * \mathrm{p} \leq .05 \quad$ n.s. $=$ nicht signifikant 



\subsection{Ingroup/Outgroup: Ähnlichkeit von Menschen mit und ohne Behinderung}

Kurzbezeichnung Variablenname

Instruktion

Quelle

Weiterführende Literatur

Skalierung (Antwortformat)

Anzahl Items

Skalenbildung

Instrument a_io_kb/b_io_kb/c_io_kb/a_io_gb/b_io_gb/

c_io_gb/a_io_pb/b_io_pb/c_io_pb

Siehe Itemwortlaut

Schubert \& Otten, 2002

Aron \& Fraley, 1999; Coats, Smith, Claypool \& Banner, 2000

Siehe Grafik auf folgender Seite: aufsteigend von 1 (oben) bis 7 (unten)

Hauptfragebogen Messzeitpunkt 1, 2 und 3

\begin{tabular}{|c|c|c|c|}
\hline \multicolumn{4}{|c|}{ Itembezeichnung } \\
\hline MZP1 & MZP2 & MZP3 & Itemwortlaut \\
\hline a_io_kb & b_io_kb & c_io_kb & $\begin{array}{l}\text { Welche Abbildung veranschaulicht deiner Meinung nach am besten, } \\
\text { wie ähnlich sich Menschen mit körperlicher Behinderung und Men- } \\
\text { schen ohne Behinderung sind? }\end{array}$ \\
\hline a_io_gb & b_io_gb & c_io_gb & $\begin{array}{l}\text { Welche Abbildung veranschaulicht deiner Meinung nach am besten, } \\
\text { wie ähnlich sich Menschen mit geistiger Behinderung und Menschen } \\
\text { ohne Behinderung sind? }\end{array}$ \\
\hline a_io_pb & b_io_pb & c_io_pb & $\begin{array}{l}\text { Welche Abbildung veranschaulicht deiner Meinung nach am besten, } \\
\text { wie ähnlich sich Menschen mit psychischer Erkrankung und Men- } \\
\text { schen ohne Behinderung sind? }\end{array}$ \\
\hline
\end{tabular}




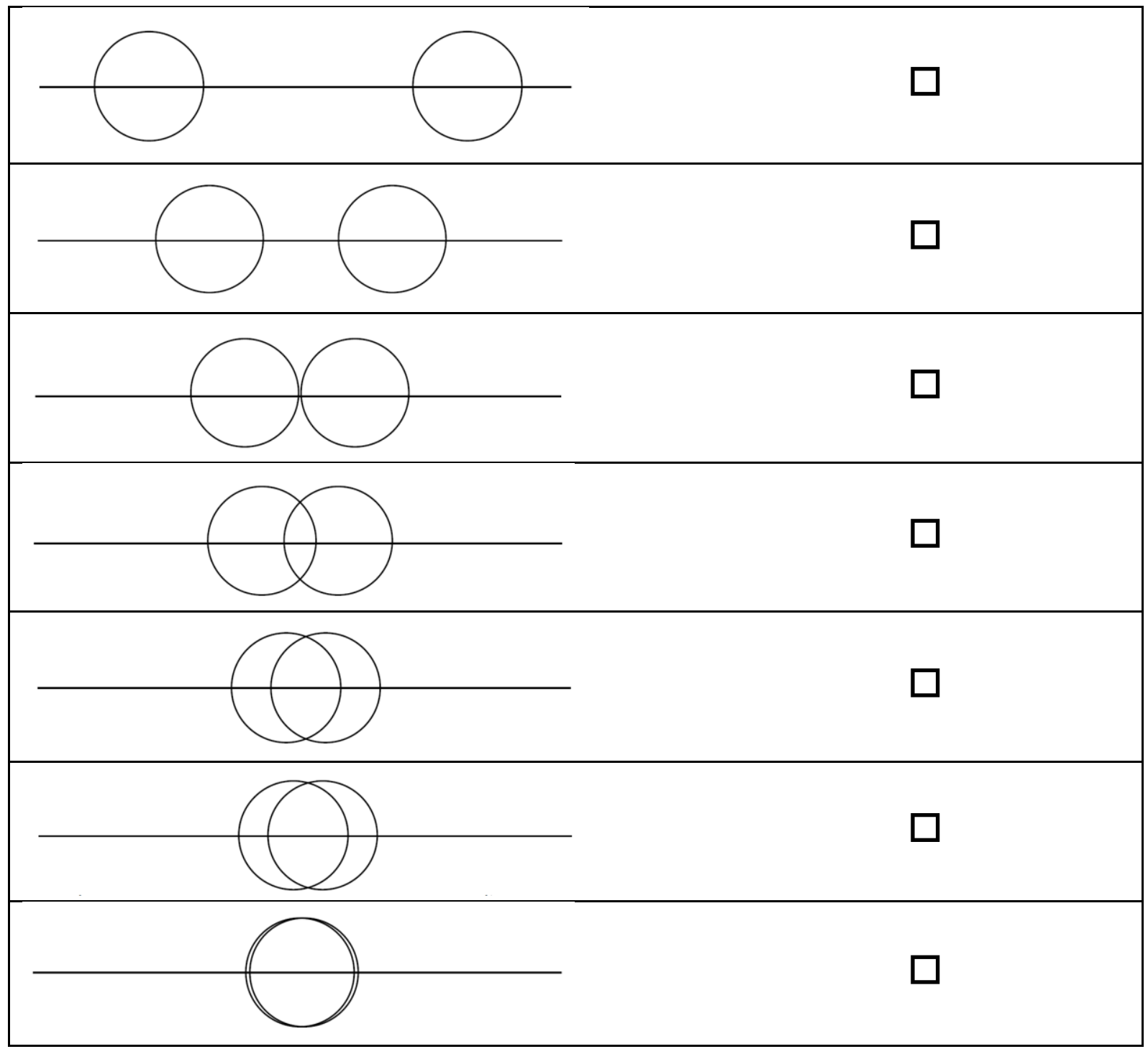

\begin{tabular}{|c|c|c|c|c|c|c|c|c|c|c|c|}
\hline \multirow{2}{*}{\multicolumn{3}{|c|}{ Itembezeichnung }} & \multicolumn{9}{|c|}{ Itemkennwerte } \\
\hline & & & \multicolumn{3}{|c|}{ MZP1 } & \multicolumn{3}{|c|}{ MZP2 } & \multicolumn{3}{|c|}{ MZP3 } \\
\hline MZP1 & MZP2 & MZP3 & MW & SD & $\mathrm{N}$ & MW & SD & $\mathrm{N}$ & MW & SD & $\mathrm{N}$ \\
\hline a_io_kb & b_io_kb & c_io_kb & 5.23 & 1.39 & 426 & 5.31 & 1.45 & 395 & 5.41 & 1.34 & 333 \\
\hline a_io_gb & b_io_gb & c_io_gb & 4.07 & 1.45 & 424 & 4.19 & 1.52 & 393 & 4.35 & 1.54 & 333 \\
\hline a_io_pb & b_io_pb & c_io_pb & 4.94 & 1.61 & 428 & 4.95 & 1.64 & 395 & 5.01 & 1.66 & 331 \\
\hline
\end{tabular}




\subsection{Soziale Distanz zu Menschen mit Behinderung}

\subsubsection{Soziale Distanz zu Menschen mit körperlicher Behinderung}

Kurzbezeichnung Variablenname

Instruktion

Quelle

Weiterführende Literatur

Skalierung (Antwortformat)

Anzahl Items

Instrument a_sd_kb/b_sd_kb/c_sd_kb

Manche Menschen sind mit einem Menschen mit Behinderung zusammen, andere wollen nicht mit ihnen in der gleichen Nachbarschaft leben. Wie ist das bei dir? Beantworte bitte spontan folgende Fragen mit Ja oder Nein.

Menschen mit körperlicher Behinderung...

Bogardus, 1933

Hodgetts \& Stolte, 2016; Wark \& Galliher, 2007

Dichotom; 0 = Nein, $1=\mathrm{Ja}$

4

Hauptfragebogen Messzeitpunkt 1, 2 und 3

\begin{tabular}{llll}
\hline \multicolumn{2}{l}{ Itembezeichnung } & & \\
MZP1 & MZP2 & MZP3 & Itemwortlaut \\
\hline a_sd_kb & b_sd_k & c_sd_k & ...kann ich mir als Partner/in (Liebesbeziehung) vorstellen. \\
1 & b1 & b1 & \\
a_sd_kb & b_sd_k & c_sd_k & $\ldots$...kann ich mir als Freund/in (Freundschaft) vorstellen. \\
2 & b2 & b2 & \\
a_sd_kb & b_sd_k & c_sd_k & $\ldots$..kann ich mir als Arbeitskolleg/in vorstellen. \\
3 & b3 & b3 & \\
a_sd_kb & b_sd_k & c_sd_k & $\ldots$ \\
4 & b4 & b4 & ...kann ich mir als Nachbar/in vorstellen. \\
\hline
\end{tabular}




\begin{tabular}{|c|c|c|c|c|c|c|}
\hline \multirow{3}{*}{$\begin{array}{l}\text { MZP1 } \\
(\mathrm{N}=445)\end{array}$} & \multirow{3}{*}{$\begin{array}{l}\text { Ausprägung } \\
0 \\
\mathrm{~N}\end{array}$} & \multirow[b]{3}{*}{$\%$} & \multirow{3}{*}{$\begin{array}{l}\text { Ausprägung } \\
1 \\
N\end{array}$} & \multicolumn{3}{|c|}{ Fehlende Werte } \\
\hline & & & & & & \\
\hline & & & & $\%$ & $\mathrm{~N}$ & $\%$ \\
\hline a_sd_kb1 & 208 & 46.74 & 204 & 45.84 & 33 & 7.42 \\
\hline a_sd_kb2 & 9 & 2.02 & 414 & 93.03 & 22 & 4.94 \\
\hline a_sd_kb3 & 10 & 2.25 & 415 & 93.26 & 20 & 4.49 \\
\hline a_sd_kb4 & 2 & 0.45 & 421 & 94.61 & 22 & 4.94 \\
\hline
\end{tabular}

\begin{tabular}{lllllll}
\hline & Ausprägung & \multicolumn{3}{c}{ Ausprägung } & \multicolumn{2}{c}{ Fehlende Werte } \\
MZP2 & 0 & & 1 & & $\mathrm{~N}$ & $\%$ \\
$(\mathrm{~N}=414)$ & $\mathrm{N}$ & $\%$ & $\mathrm{~N}$ & $\%$ & 28 & 6.76 \\
\hline b_sd_kb1 & 182 & 43.96 & 204 & 49.28 & 23 & 5.56 \\
b_sd_kb2 & 12 & 2.90 & 379 & 91.55 & 23 & 5.56 \\
b_sd_kb3 & 9 & 2.17 & 382 & 92.27 & 23 & 5.56 \\
b_sd_kb4 & 5 & 1.21 & 386 & 93.24 & 23 & \\
\hline
\end{tabular}

\begin{tabular}{lllllll}
\hline & Ausprägung & \multicolumn{3}{c}{ Ausprägung } & \multicolumn{2}{l}{ Fehlende Werte } \\
MZP3 & 0 & & 1 & & & \\
$(\mathrm{~N}=344)$ & $\mathrm{N}$ & $\%$ & $\mathrm{~N}$ & $\%$ & $\mathrm{~N}$ & $\%$ \\
\hline c_sd_kb1 & 157 & 45.64 & 172 & 50.00 & 15 & 4.36 \\
c_sd_kb2 & 18 & 5.23 & 314 & 91.28 & 12 & 3.49 \\
c_sd_kb3 & 9 & 2.62 & 322 & 93.60 & 13 & 3.78 \\
c_sd_kb4 & 3 & 0.87 & 328 & 95.35 & 13 & 3.78 \\
\hline
\end{tabular}




\subsubsection{Soziale Distanz zu Menschen mit geistiger Behinderung}

\begin{tabular}{|c|c|c|c|}
\hline \multicolumn{3}{|c|}{ Kurzbezeichnung Variablenname } & a_sd_gb/b_sd_gb/c_sd_gb \\
\hline \multicolumn{3}{|c|}{ Instruktion } & Menschen mit geistiger Behinderung... \\
\hline \multicolumn{3}{|l|}{ Quelle } & Bogardus, 1933 \\
\hline \multicolumn{3}{|c|}{ Weiterführende Literatur } & Hodgetts \& Stolte, 2016; Wark \& Galliher, 2007 \\
\hline \multicolumn{3}{|c|}{ Skalierung (Antwortformat) } & Dichotom; $0=$ Nein, $1=\mathrm{Ja}$ \\
\hline \multicolumn{3}{|c|}{ Anzahl Items } & 4 \\
\hline \multicolumn{3}{|c|}{ Instrument } & Hauptfragebogen Messzeitpunkt 1, 2 und 3 \\
\hline \multicolumn{4}{|c|}{ Itembezeichnung } \\
\hline MZP1 & MZP2 & MZP3 & Itemwortlaut \\
\hline $\begin{array}{l}\text { a_sd_gb } \\
1\end{array}$ & $\begin{array}{l}\text { b_sd_g } \\
\text { b1 }\end{array}$ & $\begin{array}{l}\text { c_sd_gb } \\
1\end{array}$ & ...kann ich mir als Partner/in (Liebesbeziehung) vorstellen. \\
\hline $\begin{array}{l}\text { a_sd_gb } \\
2\end{array}$ & $\begin{array}{l}\text { b_sd_g } \\
\text { b2 }\end{array}$ & $\begin{array}{l}\text { c_sd_gb } \\
2\end{array}$ & ...kann ich mir als Freund/in (Freundschaft) vorstellen. \\
\hline $\begin{array}{l}\text { a_sd_gb } \\
3\end{array}$ & $\begin{array}{l}\text { b_sd_g } \\
\text { b3 }\end{array}$ & $\begin{array}{l}\text { c_sd_gb } \\
3\end{array}$ & ...kann ich mir als Arbeitskolleg/in vorstellen. \\
\hline $\begin{array}{l}\text { a_sd_gb } \\
4\end{array}$ & $\begin{array}{l}\text { b_sd_g } \\
\text { b4 }\end{array}$ & $\begin{array}{l}\text { c_sd_gb } \\
4\end{array}$ & ...kann ich mir als Nachbar/in vorstellen. \\
\hline
\end{tabular}




\begin{tabular}{|c|c|c|c|c|c|c|}
\hline \multirow{3}{*}{$\begin{array}{l}\text { MZP1 } \\
(\mathrm{N}=445)\end{array}$} & \multirow{3}{*}{$\begin{array}{l}\text { Ausprägung } \\
0 \\
\mathrm{~N}\end{array}$} & \multirow[b]{3}{*}{$\%$} & \multirow{3}{*}{$\begin{array}{l}\text { Ausprägung } \\
1 \\
\mathrm{~N}\end{array}$} & \multicolumn{3}{|c|}{ Fehlende Werte } \\
\hline & & & & & & \\
\hline & & & & $\%$ & $\mathrm{~N}$ & $\%$ \\
\hline a_sd_gb1 & 381 & 85.62 & 33 & 7.42 & 31 & 6.97 \\
\hline a_sd_gb2 & 68 & 15.28 & 356 & 80.00 & 21 & 4.72 \\
\hline a_sd_gb3 & 65 & 14.61 & 358 & 80.45 & 22 & 4.94 \\
\hline a_sd_gb4 & 16 & 3.60 & 408 & 91.69 & 21 & 4.72 \\
\hline
\end{tabular}

\begin{tabular}{lllllll}
\hline & Ausprägung & \multicolumn{3}{c}{ Ausprägung } \\
MZP2 & 0 & & 1 & \multicolumn{2}{c}{ Fehlende Werte } \\
$(\mathrm{N}=414)$ & $\mathrm{N}$ & $\%$ & $\mathrm{~N}$ & $\%$ & $\mathrm{~N}$ & $\%$ \\
\hline b_sd_gb1 & 346 & 83.57 & 40 & 9.66 & 28 & 6.76 \\
b_sd_gb2 & 68 & 16.43 & 324 & 78.26 & 22 & 5.31 \\
b_sd_gb3 & 45 & 10.87 & 345 & 83.33 & 24 & 5.80 \\
b_sd_gb4 & 13 & 3.14 & 376 & 90.82 & 25 & 6.04 \\
\hline
\end{tabular}

\begin{tabular}{lllllll}
\hline & Ausprägung & \multicolumn{3}{c}{ Ausprägung } & \multicolumn{2}{l}{ Fehlende Werte } \\
MZP3 & 0 & & 1 & & & \\
(N =344) & $\mathrm{N}$ & $\%$ & $\mathrm{~N}$ & $\%$ & $\mathrm{~N}$ & $\%$ \\
\hline c_sd_gb1 & 301 & 87.50 & 28 & 8.14 & 15 & 4.36 \\
c_sd_gb2 & 75 & 21.80 & 256 & 74.42 & 13 & 3.78 \\
c_sd_gb3 & 50 & 14.53 & 281 & 81.69 & 13 & 3.78 \\
c_sd_gb4 & 10 & 2.91 & 321 & 93.31 & 13 & 3.78 \\
\hline
\end{tabular}




\subsubsection{Soziale Distanz zu Menschen mit psychischer Erkrankung}

Kurzbezeichnung Variablenname

Instruktion

Quelle

Weiterführende Literatur

Skalierung (Antwortformat)

Anzahl Items

Instrument a_sd_pb/b_sd_pb/c_sd_pb

Menschen mit psychischer Erkrankung...

Bogardus, 1933

Hodgetts \& Stolte, 2016; Wark \& Galliher, 2007

Dichotom; 0 = Nein, $1=\mathrm{Ja}$

4

Hauptfragebogen Messzeitpunkt 1, 2 und 3

\begin{tabular}{llll}
\hline \multicolumn{2}{l}{ Itembezeichnung } & & \\
MZP1 & MZP2 & MZP3 & Itemwortlaut \\
\hline a_sd_p & b_sd_P & c_sd_p & .. kann ich mir als Partner/in (Liebesbeziehung) vorstellen. \\
b1 & b1 & b1 & \\
a_sd_p & b_sd_P & c_sd_p & ..kann ich mir als Freund/in (Freundschaft) vorstellen. \\
b2 & b2 & b2 & \\
a_sd_P & b_sd_P & c_sd_P & ...kann ich mir als Arbeitskolleg/in vorstellen. \\
b3 & b3 & b3 & \\
a_sd_p & b_sd_P & c_sd_P & ...kann ich mir als Nachbar/in vorstellen. \\
b4 & b4 & b4 & \\
\hline
\end{tabular}




\begin{tabular}{lllllll}
\hline & Ausprägung & \multicolumn{3}{c}{ Ausprägung } \\
MZP1 & 0 & & 1 & \multicolumn{2}{c}{ Fehlende Werte } \\
(N = 445) & $\mathrm{N}$ & $\%$ & $\mathrm{~N}$ & $\%$ & $\mathrm{~N}$ & $\%$ \\
\hline a_sd_pb1 & 214 & 48.09 & 196 & 44.04 & 35 & 7.87 \\
a_sd_pb2 & 46 & 10.34 & 377 & 84.72 & 22 & 4.94 \\
a_sd_pb3 & 41 & 9.21 & 380 & 85.39 & 24 & 5.39 \\
a_sd_pb4 & 19 & 4.27 & 405 & 91.01 & 21 & 4.72 \\
\hline
\end{tabular}

\begin{tabular}{lllllll}
\hline & Ausprägung & \multicolumn{3}{c}{ Ausprägung } \\
MZP2 & 0 & & 1 & \multicolumn{2}{c}{ Fehlende Werte } \\
$(\mathrm{N}=414)$ & $\mathrm{N}$ & $\%$ & $\mathrm{~N}$ & $\%$ & $\mathrm{~N}$ & $\%$ \\
\hline b_sd_pb1 & 208 & 50.24 & 177 & 42.75 & 29 & 7.00 \\
b_sd_pb2 & 40 & 9.66 & 350 & 84.54 & 24 & 5.80 \\
b_sd_pb3 & 40 & 9.66 & 352 & 85.02 & 22 & 5.31 \\
b_sd_pb4 & 22 & 5.31 & 370 & 89.37 & 22 & 5.31 \\
\hline
\end{tabular}

\begin{tabular}{lllllll}
\hline & Ausprägung & \multicolumn{3}{c}{ Ausprägung } & \multicolumn{2}{l}{ Fehlende Werte } \\
MZP3 & 0 & & 1 & $\%$ & $\mathrm{~N}$ & $\%$ \\
(N =344) & $\mathrm{N}$ & $\%$ & $\mathrm{~N}$ & 42.15 & 18 & 5.23 \\
\hline c_sd_pb1 & 181 & 52.62 & 145 & 82.27 & 12 & 3.49 \\
c_sd_pb2 & 49 & 14.24 & 283 & 87.79 & 12 & 3.49 \\
c_sd_pb3 & 30 & 8.72 & 302 & 92.73 & 12 & 3.49 \\
c_sd_pb4 & 13 & 3.78 & 319 & & & \\
\hline
\end{tabular}




\subsection{Multidimensional Attitudes Scale (MAS)}

\subsubsection{Affektive Dimension}

Kurzbezeichnung Variablenname

Instruktion

Quelle

Weiterführende Literatur

Skalierung (Antwortformat)

Anzahl Items

Instrument

\author{
a_maa/b_maa/c_maa
}

Du gehst in der Mittagspause mit einem Freund in der Stadt etwas essen. Ein junger Mann im Rollstuhl kommt in das Lokal. Er kennt deinen Freund, weil er in der gleichen Straße wohnt, und gesellt sich zu euch. Du bemerkst, dass er auch seine Arme nur eingeschränkt bewegen kann und Schwierigkeiten beim Sprechen hat. Kurz darauf muss dein Freund los, aber du bist noch nicht fertig mit deinem Essen. Man kann ganz verschiedene Gefühle in so einer Situation haben. Bitte gib an, wie du dich in der Situation am ehesten fühlst. Ich fühle mich...

Adaptiert von Findler et al., 2007

Bolt, 2014; Werth \& Mayer, 2008

Semantisches Differential mit Endpolbeschriftung 1 (...) bis $6(\ldots)$, siehe folgende Tabelle

18

Hauptfragebogen Messzeitpunkt 1, 2 und 3

\begin{tabular}{llll}
\hline \multicolumn{2}{l}{ Itembezeichnung } & & \\
MZP1 & MZP2 & MZP3 & Itemwortlaut \\
\hline a_maa1 & b_maa1 & c_maa1 & angespannt $[\ldots]$ entspannt \\
a_maa2 & b_maa2 & c_maa2 & hilflos $[\ldots]$ souverän \\
a_maa3 & b_maa3 & c_maa3 & nervös $[\ldots]$ gelassen \\
a_maa4 & b_maa4 & c_maa4 & gefangen $[\ldots]$ frei \\
a_maa5 & b_maa5 & c_maa5 & unruhig $[\ldots]$ ruhig \\
a_maa6 & b_maa6 & c_maa6 & deprimiert $[\ldots]$ unbekümmert \\
a_maa7 & b_maa7 & c_maa7 & unzufrieden $[\ldots]$ zufrieden \\
a_maa8 & b_maa8 & c_maa8 & unsicher $[\ldots]$ sicher \\
a_maa9 & b_maa9 & c_maa9 & unterlegen $[\ldots]$ überlegen \\
\hline
\end{tabular}




\begin{tabular}{llll}
\hline \multicolumn{2}{l}{ Itembezeichnung } & & \\
MZP1 & MZP2 & MZP3 & Itemwortlaut \\
\hline a_maa10 & b_maa10 & c_maa10 & mitleidig $[\ldots]$ ebenbürtig \\
a_maa11 & b_maa11 & c_maa11 & abgestoßen $[\ldots]$ angezogen \\
a_maa12 & b_maa12 & c_maa12 & gelangweilt $[\ldots . .$.$] aktiviert$ \\
a_maa13 & b_maa13 & c_maa13 & belastet $[\ldots]$ ausgeglichen \\
a_maa14 & b_maa14 & c_maa14 & demütig $[\ldots]$ stolz \\
a_maa15 & b_maa15 & c_maa15 & befangen $[\ldots]$ unbefangen \\
a_maa16 & b_maa16 & c_maa16 & verärgert $[\ldots]$ erfreut \\
a_maa17 & b_maa17 & c_maa17 & fremd $[\ldots]$ vertraut \\
a_maa18 & b_maa18 & c_maa18 & verängstigt $[\ldots]$ ermutigt \\
\hline
\end{tabular}

\begin{tabular}{|c|c|c|c|c|c|c|c|c|}
\hline \multicolumn{3}{|c|}{ Itembezeichnung } & \multicolumn{2}{|c|}{ MZP1 $(\mathrm{N}=360)$} & \multicolumn{2}{|c|}{ MZP2 $(\mathrm{N}=345)$} & \multicolumn{2}{|c|}{ MZP3 $(N=293)$} \\
\hline MZP1 & MZP2 & MZP3 & MW & $\mathrm{SD}$ & MW & $\mathrm{SD}$ & MW & SD \\
\hline a_maa1 & b_maa1 & c_maa1 & 3.81 & 1.48 & 4.06 & 1.45 & 4.28 & 1.35 \\
\hline a_maa2 & b_maa2 & c_maa2 & 4.03 & 1.26 & 4.20 & 1.25 & 4.37 & 1.19 \\
\hline a_maa3 & b_maa3 & c_maa3 & 3.76 & 1.48 & 4.07 & 1.42 & 4.19 & 1.37 \\
\hline a_maa4 & b_maa4 & c_maa4 & 4.27 & 1.34 & 4.41 & 1.34 & 4.41 & 1.30 \\
\hline a_maa5 & b_maa5 & c_maa5 & 4.24 & 1.32 & 4.33 & 1.33 & 4.41 & 1.37 \\
\hline a_maa6 & b_maa6 & c_maa6 & 4.63 & 1.07 & 4.62 & 1.14 & 4.73 & 1.06 \\
\hline a_maa7 & b_maa7 & c_maa7 & 4.46 & 1.17 & 4.51 & 1.22 & 4.45 & 1.26 \\
\hline a_maa8 & b_maa8 & c_maa8 & 3.73 & 1.55 & 3.98 & 1.53 & 4.21 & 1.39 \\
\hline a_maa9 & b_maa9 & c_maa9 & 3.94 & .87 & 3.96 & .89 & 3.97 & .87 \\
\hline a_maa10 & b_maa10 & c_maa10 & 4.14 & 1.44 & 4.18 & 1.44 & 4.31 & 1.33 \\
\hline a_maa11 & b_maa11 & c_maa11 & 4.05 & .92 & 4.02 & .90 & 3.96 & .93 \\
\hline a_maa12 & b_maa12 & c_maa12 & 4.66 & 1.07 & 4.57 & 1.04 & 4.38 & 1.24 \\
\hline a_maa13 & b_maa13 & c_maa13 & 4.19 & 1.29 & 4.30 & 1.21 & 4.38 & 1.30 \\
\hline a_maa14 & b_maa14 & c_maa14 & 3.91 & 1.08 & 3.87 & .94 & 4.04 & 1.03 \\
\hline a_maa15 & b_maa15 & c_maa15 & 4.13 & 1.30 & 4.25 & 1.28 & 4.28 & 1.23 \\
\hline a_maa16 & b_maa16 & c_maa16 & 4.36 & 1.21 & 4.36 & 1.20 & 4.40 & 1.23 \\
\hline a_maa17 & b_maa17 & c_maa17 & 3.22 & 1.42 & 3.39 & 1.44 & 3.49 & 1.41 \\
\hline a_maa18 & b_maa18 & c_maa18 & 4.18 & 1.17 & 4.24 & 1.13 & 4.28 & 1.15 \\
\hline
\end{tabular}




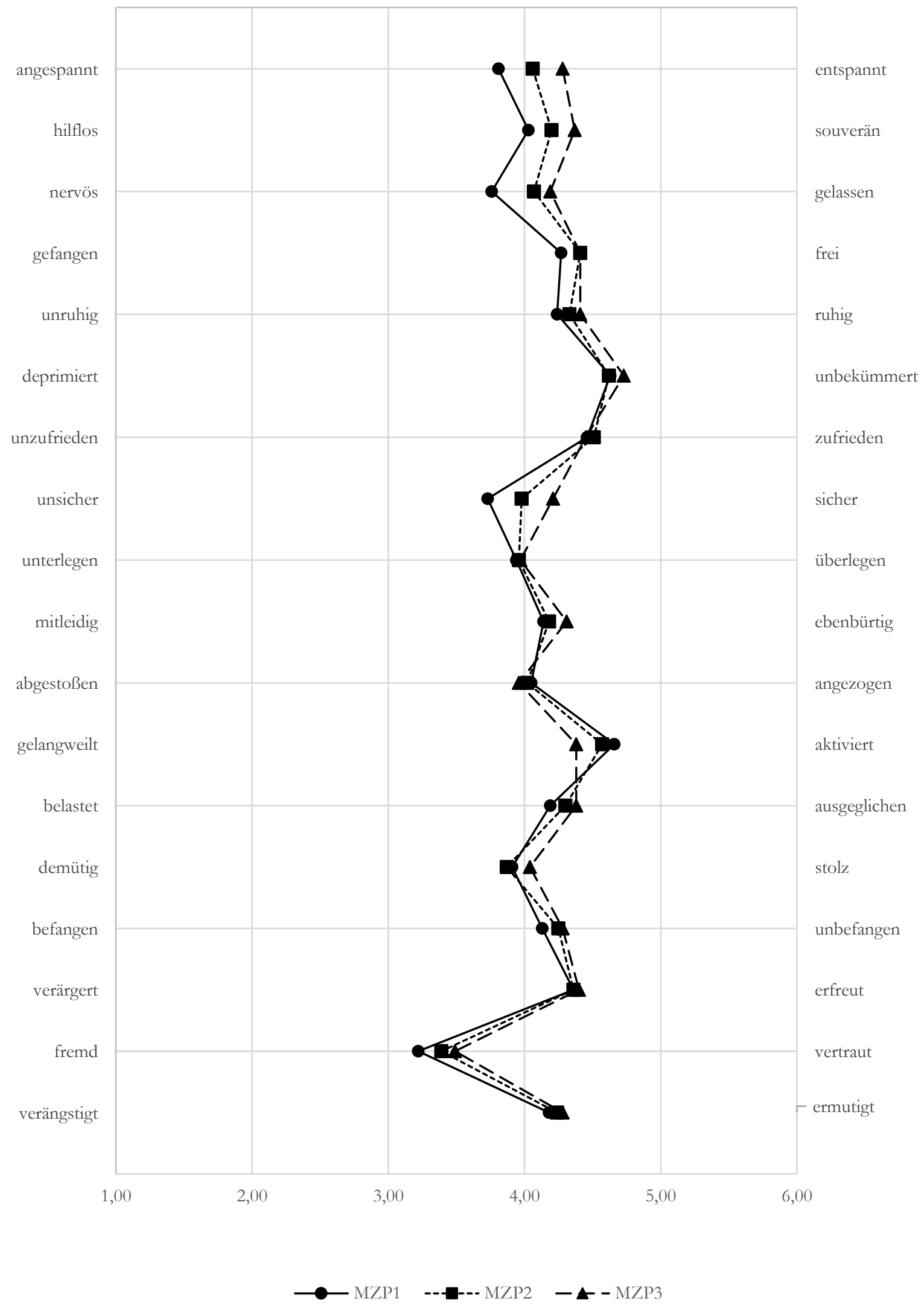





\subsubsection{Kognitive Dimension}

Kurzbezeichnung Variablenname

Instruktion

Quelle

Weiterführende Literatur

Skalierung (Antwortformat)

Inverse Items (-)

Anzahl Items

Instrument

$$
\text { a_mak/b_mak/c_mak }
$$

Man kann ganz verschiedene Gedanken in so einer Situation haben. Bitte gib zu jedem Gedanken an, wie wahrscheinlich es ist, dass er dir in der Situation kommt.

Adaptiert von Findler et al., 2007

Bolt, 2014; Werth \& Mayer, 2008

Endpolbeschriftung; 1 = wahrscheinlich überhaupt nicht, $(\ldots), 6=$ sehr wahrscheinlich

a_mak2, b_mak2, c_mak2, a_mak3, b_mak3, c_mak3, a_mak5, b_mak5, c_mak5, a_mak7, b_mak7, c_mak7, a_mak10, b_mak10, c_mak10, a_mak14, b_mak14, c_mak14, a_mak15, b_mak15, c_mak15, a_mak16, b_mak16, c_mak16, a_mak19, b_mak19, c_mak19

19

Hauptfragebogen Messzeitpunkt 1, 2 und 3

\begin{tabular}{llll}
\hline $\begin{array}{l}\text { Itembezeichnung } \\
\text { MZP1 }\end{array}$ & MZP2 & MZP3 & Itemwortlaut \\
\hline a_mak1 & b_mak1 & c_mak1 & Er scheint ein interessanter Typ zu sein. \\
a_mak2 & b_mak2 & c_mak2 & $\begin{array}{l}\text { Ich hoffe, irgendetwas unterbricht die Situation schnell. (-) } \\
\text { a_mak3 }\end{array}$ \\
b_mak3 & c_mak3 & $\begin{array}{l}\text { Mir muss schnell eine Ausrede einfallen, warum ich plötzlich gehen } \\
\text { muss. (-) }\end{array}$ \\
a_mak4 & b_mak4 & c_mak4 & Ich treffe gern neue Menschen. \\
a_mak5 & b_mak5 & c_mak5 & Ich habe überhaupt keine Lust, mich mit ihm zu unterhalten. (-) \\
a_mak6 & b_mak6 & c_mak6 & Wir finden bestimmt Gesprächsthemen, die uns beide interessieren. \\
a_mak7 & b_mak7 & c_mak7 & Ich hoffe, er sagt von sich aus, dass er jetzt los muss. (-) \\
a_mak8 & b_mak8 & c_mak8 & Ist doch nett ihn besser kennenzulernen. \\
a_mak9 & b_mak9 & c_mak9 & Er findet es bestimmt gut, wenn ich ein Gespräch beginne. \\
a_mak10 & b_mak10 & c_mak10 & Irgendwie werde ich die Situation schon überleben. (-) \\
a_mak11 & b_mak11 & c_mak11 & Der sieht doch ganz nett aus. \\
\hline
\end{tabular}




\begin{tabular}{|c|c|c|c|}
\hline \multicolumn{4}{|c|}{ Itembezeichnung } \\
\hline MZP1 & MZP2 & MZP3 & Itemwortlaut \\
\hline a_mak12 & b_mak12 & c_mak12 & Er freut sich bestimmt, mich kennenzulernen. \\
\hline a_mak13 & b_mak13 & c_mak13 & $\begin{array}{l}\text { Hoffentlich fällt ihm ein gutes Thema ein, über das wir uns unterhalten } \\
\text { können. }\end{array}$ \\
\hline a_mak14 & b_mak14 & c_mak14 & Ein Gespräch mit ihm wird sicherlich ziemlich anstrengend werden. (-) \\
\hline a_mak15 & b_mak15 & c_mak15 & Ich weiß nicht, worüber wir uns unterhalten sollten. (-) \\
\hline a_mak16 & b_mak16 & c_mak16 & Hoffentlich kennt er hier noch andere Menschen. (-) \\
\hline a_mak17 & b_mak17 & c_mak17 & Er sieht freundlich aus. \\
\hline a_mak18 & b_mak18 & c_mak18 & Ich werde dafür sorgen, dass er sich wohlfühlt. \\
\hline a_mak19 & b_mak19 & c_mak19 & Ich würde jetzt am liebsten auch direkt gehen. (-) \\
\hline
\end{tabular}

\begin{tabular}{lllllllll}
\multicolumn{2}{c}{ Itembezeichnung } & & \multicolumn{2}{c}{ MZP1 } & $(\mathrm{N}=401)$ & \multicolumn{2}{c}{ MZP2 $(\mathrm{N}=367)$} & \multicolumn{2}{c}{ MZP3 (N $=305)$} \\
MZP1 & MZP2 & MZP3 & MW & SD & MW & SD & MW & SD \\
\hline a_mak1 & b_mak1 & c_mak1 & 4.35 & 1.22 & 4.27 & 1.19 & 4.31 & 1.22 \\
ra_mak2 & rb_mak2 & rc_mak2 & 4.53 & 1.32 & 4.40 & 1.33 & 4.50 & 1.37 \\
ra_mak3 & rb_mak3 & rc_mak3 & 5.12 & 1.11 & 4.90 & 1.21 & 4.83 & 1.28 \\
a_mak4 & b_mak4 & c_mak4 & 4.51 & 1.43 & 4.57 & 1.41 & 4.45 & 1.43 \\
ra_mak5 & rb_mak5 & rc_mak5 & 5.15 & 1.07 & 4.89 & 1.16 & 4.82 & 1.24 \\
a_mak6 & b_mak6 & c_mak6 & 4.78 & 1.20 & 4.64 & 1.18 & 4.71 & 1.24 \\
ra_mak7 & rb_mak7 & rc_mak7 & 4.73 & 1.36 & 4.55 & 1.40 & 4.46 & 1.45 \\
a_mak8 & b_mak8 & c_mak8 & 4.73 & 1.18 & 4.63 & 1.21 & 4.53 & 1.21 \\
a_mak9 & b_mak9 & c_mak9 & 4.62 & 1.18 & 4.52 & 1.17 & 4.40 & 1.21 \\
ra_mak10 & rb_mak10 & rc_mak10 & 3.42 & 1.79 & 3.56 & 1.76 & 3.65 & 1.75 \\
a_mak11 & b_mak11 & c_mak11 & 4.79 & 1.12 & 4.66 & 1.14 & 4.69 & 1.21 \\
a_mak12 & b_mak12 & c_mak12 & 4.45 & 1.30 & 4.28 & 1.27 & 4.26 & 1.35 \\
a_mak13 & b_mak13 & c_mak13 & 4.14 & 1.30 & 3.97 & 1.34 & 3.89 & 1.34 \\
ra_mak14 & rb_mak14 & rc_mak14 & 4.71 & 1.20 & 4.64 & 1.24 & 4.69 & 1.28 \\
ra_mak15 & rb_mak15 & rc_mak15 & 3.71 & 1.53 & 3.57 & 1.46 & 3.74 & 1.46 \\
ra_mak16 & rb_mak16 & rc_mak16 & 4.66 & 1.28 & 4.62 & 1.31 & 4.63 & 1.29 \\
a_mak17 & b_mak17 & c_mak17 & 4.97 & .96 & 4.89 & 1.01 & 4.78 & 1.15 \\
a_mak18 & b_mak18 & c_mak18 & 4.61 & 1.25 & 4.58 & 1.22 & 4.42 & 1.34 \\
ra_mak19 & rb_mak19 & rc_mak19 & 4.98 & 1.27 & 4.78 & 1.40 & 4.81 & 1.39 \\
\hline & & & & & & & &
\end{tabular}


Er scheint ein interessanter Typ zu sein.

Ich hoffe, irgendetwas unterbricht die Situation

Mir muss schnell eine Ausrede einfallen, warum ich plötzlich gehen muss.

Ich treffe gern neue Menschen.

Ich habe überhaupt keine

Lust, mich mit ihm zu unterhalten. (-)

Wir finden bestimmt Gesprächsthemen, die uns beide interessieren. Ich hoffe, er sagt von sich aus, dass er jetzt los muss.

Ist doch nett ihn besser kennenzulernen.

Er findet es bestimmt gut, wenn ich ein Gespräch beginne.

Irgendwie werde ich die Situation schon überleben.

Der sieht doch ganz nett aus.

Er freut sich bestimmt, mich kennenzulernen.

Hoffentlich fällt ihm ein gutes Thema ein, über das wir uns unterhalten

Ein Gespräch mönnen. wird sicherlich ziemlich anstrengend werden. (-) Ich weiß nicht, worüber wir uns unterhalten sollten. (-)

Hoffentlich kennt er hier noch andere Menschen. (-)

Er sieht freundlich aus.

Ich werde dafür sorgen, dass er sich wohlfühlt.

Ich würde jetzt am liebsten auch direkt gehen.

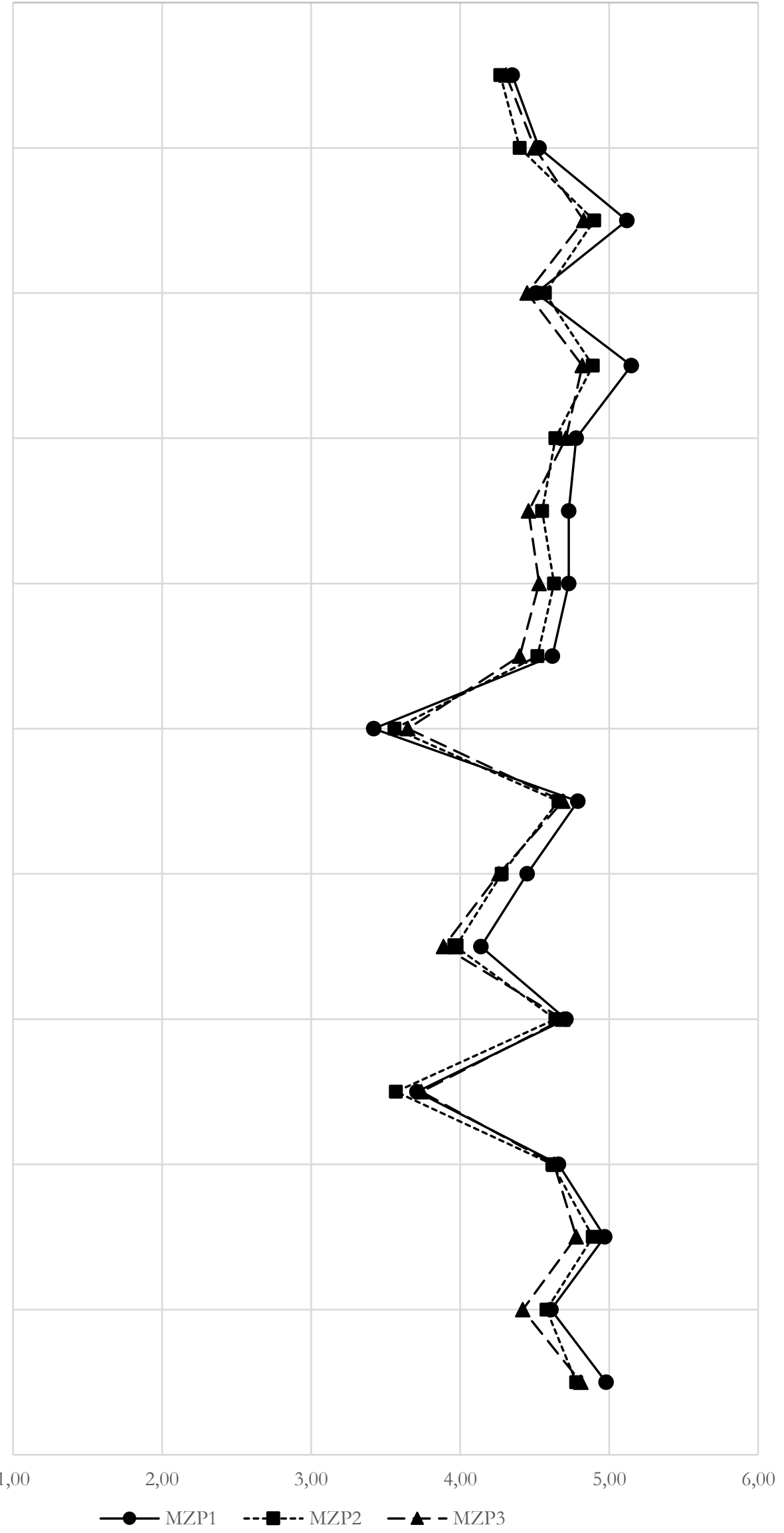





\subsubsection{Behaviorale Dimension}

Kurzbezeichnung Variablenname

Instruktion

Quelle

Weiterführende Literatur

Skalierung (Antwortformat)

Inverse Items (-)

Anzahl Items

Instrument a_mab/b_mab/c_mab

Man kann sich in so einer Situation ganz unterschiedlich verhalten. Bitte gib an, wie wahrscheinlich es ist, dass du dich in der Situation so verhältst. Dieses Verhalten wäre...

Adaptiert von Findler et al., 2007

Bolt, 2014; Werth \& Mayer, 2008

Endpolbeschriftung; 1 = wahrscheinlich überhaupt nicht, $(\ldots), 6=$ sehr wahrscheinlich

a_mab1, b_mab1, c_mab1, a_mab2, b_mab2,

c_mab2, a_mab3, b_mab3, c_mab3, a_mab4,

b_mab4, c_mab4, a_mab7, b_mab7, c_mab7,

a_mab8, b_mab8, c_mab8, a_mab10, b_mab10,

c_mab10,a_mab11, b_mab11, c_mab11

12

Hauptfragebogen Messzeitpunkte 1, 2 und 3

\begin{tabular}{llll}
\hline \multicolumn{2}{l}{ Itembezeichnung } & & \\
MZP1 & MZP2 & MZP3 & Itemwortlaut \\
\hline a_mab1 & b_mab1 & c_mab1 & Aufstehen und gehen. (-) \\
a_mab2 & b_mab2 & c_mab2 & Eine Zeitschrift lesen oder mich mit meinem Handy beschäftigen. (-) \\
a_mab3 & b_mab3 & c_mab3 & Eine Ausrede erfinden, um gehen zu können. (-) \\
a_mab4 & b_mab4 & c_mab4 & An einen anderen Tisch setzen. (-) \\
a_mab5 & b_mab5 & c_mab5 & Von mir aus ein Gespräch beginnen. \\
a_mab6 & b_mab6 & c_mab6 & Ein Gespräch beginnen, falls er nicht damit anfängt. \\
a_mab7 & b_mab7 & c_mab7 & Auf Toilette gehen und ein wenig Zeit verstreichen lassen. (-) \\
a_mab8 & b_mab8 & c_mab8 & Die Speisekarte durchblättern und nach etwas suchen. (-) \\
a_mab9 & b_mab9 & c_mab9 & Ihn zum Essen einladen. \\
a_mab10 & b_mab10 & c_mab10 & Schnell aufessen, damit ich schnell gehen kann. (-) \\
a_mab11 & b_mab11 & c_mab11 & $\begin{array}{l}\text { Mich umsehen, ob ich jemand anderes entdecke, mit dem ich mich } \\
\text { unterhalten kann. (-) }\end{array}$ \\
a_mab12 & b_mab12 & c_mab12 & Ihn fragen, ob er Hilfe braucht. \\
\hline
\end{tabular}




\begin{tabular}{lllllllll}
\hline \multicolumn{2}{c}{ Itembezeichnung } & & \multicolumn{2}{c}{ MZP1 $(\mathrm{N}=431)$} & \multicolumn{2}{c}{ MZP2 $(\mathrm{N}=390)$} & \multicolumn{2}{c}{ MZP3 (N = 328) } \\
MZP1 & MZP2 & MZP3 & MW & SD & MW & SD & MW & SD \\
\hline ra_mab1 & rb_mab1 & rc_mab1 & 5.65 & .85 & 5.56 & .98 & 5.58 & .88 \\
ra_mab2 & rb_mab2 & rc_mab2 & 4.88 & 1.29 & 4.81 & 1.34 & 4.78 & 1.36 \\
ra_mab3 & rb_mab3 & rc_mab3 & 5.15 & 1.21 & 5.06 & 1.27 & 4.94 & 1.32 \\
ra_mab4 & rb_mab4 & rc_mab4 & 5.87 & .46 & 5.72 & .82 & 5.72 & .79 \\
a_mab5 & b_mab5 & c_mab5 & 4.63 & 1.13 & 4.57 & 1.18 & 4.56 & 1.15 \\
a_mab6 & b_mab6 & c_mab6 & 4.85 & 1.12 & 4.83 & 1.20 & 4.81 & 1.17 \\
ra_mab7 & rb_mab7 & rc_mab7 & 5.00 & 1.24 & 4.89 & 1.35 & 4.86 & 1.35 \\
ra_mab8 & rb_mab8 & rc_mab8 & 4.81 & 1.32 & 4.54 & 1.47 & 4.63 & 1.44 \\
a_mab9 & b_mab9 & c_mab9 & 2.74 & 1.58 & 2.67 & 1.53 & 2.49 & 1.46 \\
ra_mab10 & rb_mab10 & rc_mab10 & 5.03 & 1.19 & 4.91 & 1.31 & 4.80 & 1.35 \\
ra_mab11 & rb_mab11 & rc_mab11 & 5.45 & .87 & 5.30 & 1.07 & 5.30 & 1.04 \\
a_mab12 & b_mab12 & c_mab12 & 4.10 & 1.52 & 3.97 & 1.54 & 3.68 & 1.54 \\
\hline
\end{tabular}


Aufstehen und gehen. (-

Eine Zeitschrift lesen oder mich mit meinem Handy beschäftigen. (-)

Eine Ausrede erfinden, um gehen zu können. (-)

An einen anderen Tisch setzen. (-)

Von mir aus ein Gespräch beginnen.

Ein Gespräch beginnen, falls er nicht damit anfängt.

Auf Toilette gehen und ein wenig Zeit verstreichen lassen. (-)

Die Speisekarte durchblättern und nach etwas suchen. (-)

Ihn zum Essen einladen.

Schnell aufessen, damit ich schnell gehen kann.

Mich umsehen, ob ich jemand anderes entdecke, mit dem ich mich unterhalten kann.

Ihn fragen, ob er Hilfe braucht. 



\section{Einstellungen zu Inklusion}

\subsection{Befürwortung von Inklusion im Sinne der UN-BRK}

\subsubsection{Teilhabe}

Kurzbezeichnung Variablenname

Instruktion

Quelle

Weiterführende Literatur

Skalierung (Antwortformat)

Inverse Items (-)

Anzahl Items

Skalenbildung

Instrument xa_brk_t/xb_brk_t/xc_brk_t

(xa_brk_Teil/xb_brk_Teil/xc_brk_Teil)

Es folgt nun eine Reihe von verschiedenen Meinungen und möglichen Reaktionen zu Menschen mit Behinderung. Bitte gibt zu jeder Aussage an, inwieweit du ihr zustimmst.

Eigenentwicklung basierend auf der UN-

Behindertenrechtskonvention (2009)

Degener \& Diehl, 2015

Endpolbeschriftung; 1 = stimme überhaupt nicht zu, (...), 6 = stimme voll $\mathrm{zu}$

per Mittelwert

Hauptfragebogen Messzeitpunkt 1, 2 und 3

Itembezeichnung

\begin{tabular}{|c|c|c|c|}
\hline MZP1 & MZP2 & MZP3 & Itemwortlaut \\
\hline a_brk2 & b_brk2 & c_brk2 & $\begin{array}{l}\text { Man sollte für Menschen mit Behinderung Möglichkeiten schaffen, ihr } \\
\text { kreatives und intellektuelles Potenzial zu entfalten und zu nutzen. }\end{array}$ \\
\hline a_brk3 & b_brk3 & c_brk3 & $\begin{array}{l}\text { Menschen mit Behinderung sollte die Teilnahme an Freizeitaktivitäten } \\
\text { gleichberechtigt mit anderen ermöglicht werden. }\end{array}$ \\
\hline a_brk4 & b_brk4 & c_brk4 & $\begin{array}{l}\text { Es ist Aufgabe des Staates, sich für die Entwicklung von Produkten einzu- } \\
\text { setzen, die für Menschen mit Behinderung leicht bedienbar sind. }\end{array}$ \\
\hline a_brk8 & b_brk8 & c_brk8 & $\begin{array}{l}\text { Es sollte Kampagnen zur Förderung einer positiveren Wahrnehmung von } \\
\text { Menschen mit Behinderungen geben. }\end{array}$ \\
\hline a_brk19 & b_brk19 & c_brk19 & $\begin{array}{l}\text { Der Staat sollte sich um Familien, in denen ein Kind behindert ist, ganz } \\
\text { besonders kümmern. }\end{array}$ \\
\hline
\end{tabular}




\begin{tabular}{|c|c|c|c|c|c|c|c|c|c|c|c|c|c|c|}
\hline \multirow{2}{*}{\multicolumn{3}{|c|}{ Itembezeichnung }} & \multicolumn{12}{|c|}{ Itemkennwerte } \\
\hline & & & \multicolumn{4}{|c|}{ MZP1 } & \multicolumn{4}{|c|}{ MZP2 } & \multicolumn{4}{|c|}{ MZP3 } \\
\hline MZP1 & MZP2 & MZP3 & MW & SD & $\mathrm{r}_{\mathrm{it}}$ & $\lambda_{\mathrm{ij}}$ & MW & SD & $\mathrm{r}_{\mathrm{it}}$ & $\lambda_{\mathrm{ij}}$ & MW & SD & $r_{i t}$ & $\lambda_{\mathrm{ij}}$ \\
\hline a_brk2 & b_brk2 & c_brk2 & 5.58 & .73 & .58 & $.76^{* *}$ & 5.41 & .85 & .65 & $.69 * *$ & 5.40 & .99 & .66 & $.73 * *$ \\
\hline a_brk3 & b_brk3 & c_brk3 & 5.36 & .93 & .53 & $.70^{* *}$ & 5.31 & 1.02 & .58 & $.75^{* *}$ & 5.24 & 1.08 & .67 & $.78^{* *}$ \\
\hline a_brk4 & b_brk4 & c_brk4 & 5.06 & 1.09 & .49 & $.61 * *$ & 5.07 & 1.07 & .64 & $.69 * *$ & 5.07 & 1.14 & .67 & $.78^{* *}$ \\
\hline a_brk8 & b_brk8 & c_brk8 & 5.19 & 1.01 & .50 & $.56^{* *}$ & 5.08 & 1.08 & .60 & $.73^{* *}$ & 5.07 & 1.06 & .68 & $.74 * *$ \\
\hline a_brk19 & b_brk19 & c_brk19 & 4.40 & 1.24 & .36 & $.38^{* *}$ & 4.45 & 1.19 & .43 & $.47 * *$ & 4.38 & 1.21 & .43 & $.46^{* *}$ \\
\hline \multirow{4}{*}{\multicolumn{3}{|c|}{ Skalenkennwerte }} & \multicolumn{2}{|c|}{ Cronbachs $\alpha$} & \multicolumn{2}{|l|}{.74} & \multicolumn{2}{|c|}{ Cronbachs $\alpha$} & \multicolumn{2}{|l|}{.80} & \multicolumn{2}{|c|}{ Cronbachs $\alpha$} & \multicolumn{2}{|l|}{.83} \\
\hline & & & \multicolumn{2}{|l|}{ MW } & \multicolumn{2}{|l|}{5.11} & \multicolumn{2}{|l|}{ MW } & \multicolumn{2}{|l|}{5.07} & \multicolumn{2}{|l|}{ MW } & \multicolumn{2}{|l|}{5.03} \\
\hline & & & \multicolumn{2}{|l|}{ SD } & \multicolumn{2}{|l|}{.70} & \multicolumn{2}{|l|}{ SD } & \multicolumn{2}{|l|}{.78} & \multicolumn{2}{|l|}{ SD } & \multicolumn{2}{|l|}{.84} \\
\hline & & & \multicolumn{2}{|l|}{$\mathrm{N}$} & \multicolumn{2}{|l|}{441} & \multicolumn{2}{|l|}{$\mathrm{N}$} & \multicolumn{2}{|l|}{406} & \multicolumn{2}{|l|}{$\mathrm{N}$} & \multicolumn{2}{|l|}{340} \\
\hline & & & \multicolumn{2}{|c|}{$\chi^{2}[\mathrm{df}]$} & \multicolumn{2}{|c|}{$15.44^{* *}[5]$} & \multicolumn{2}{|c|}{$\chi^{2}[\mathrm{df}]$} & 6.77 & & $\chi^{2}[\mathrm{~d} f$ & & 16.7 & \\
\hline & & & CFI & & .98 & & CFI & & 1.00 & & CFI & & .98 & \\
\hline Messmo & & & TLI & & .95 & & TLI & & .99 & & TLI & & .96 & \\
\hline & & & RMSI & & .07 n.s. & & RMS & & $.03 \mathrm{n}$ & & RMS & & $.08 \mathrm{r}$ & \\
\hline & & & SRMF & & .03 & & SRM & & .02 & & SRM & & .03 & \\
\hline
\end{tabular}

$* * \mathrm{p} \leq .01 \quad * \mathrm{p} \leq .05 \quad$ n.s. $=$ nicht signifikant 


\subsubsection{Selbstbestimmung}

Kurzbezeichnung Variablenname

Instruktion

Quelle

Weiterführende Literatur

Skalierung (Antwortformat)

Inverse Items (-)

Anzahl Items

Skalenbildung

Instrument xa_brk_s/xb_brk_s/xc_brk_s

(xa_brk_Selbst/xb_brk_Selbst/xc_brk_Selbst)

Wie sehr stimmst du folgenden Aussagen zu?

Eigenentwicklung basierend auf der UN-

Behindertenrechtskonvention (2009)

Degener \& Diehl, 2015

Endpolbeschriftung; 1 = stimme überhaupt nicht zu, $(\ldots), 6=$ stimme voll $\mathrm{zu}$

5

per Mittelwert

Hauptfragebogen Messzeitpunkt 1, 2 und 3

\begin{tabular}{|c|c|c|c|}
\hline \multicolumn{4}{|c|}{ Itembezeichnung } \\
\hline MZP1 & MZP2 & MZP3 & Itemwortlaut \\
\hline a_brk14 & b_brk14 & c_brk14 & $\begin{array}{l}\text { Menschen mit Behinderung sollten selbstbestimmt entscheiden dürfen, an } \\
\text { welchem Ort sie leben möchten. }\end{array}$ \\
\hline a_brk15 & b_brk15 & c_brk15 & $\begin{array}{l}\text { Menschen mit Behinderung sollten über ihr privates Eigentum selbstbe- } \\
\text { stimmt verfügen dürfen. }\end{array}$ \\
\hline a_brk16 & b_brk16 & c_brk16 & $\begin{array}{l}\text { Menschen mit Behinderung sollten ein Recht auf individuelle Assistenz } \\
\text { haben, um in einer eigenen Wohnung leben zu können. }\end{array}$ \\
\hline a_brk18 & b_brk18 & c_brk18 & $\begin{array}{l}\text { Menschen mit Behinderung sollten frei entscheiden dürfen, ob und wie } \\
\text { viele Kinder sie bekommen möchten. }\end{array}$ \\
\hline a_brk21 & b_brk21 & c_brk 21 & $\begin{array}{l}\text { Der Staat sollte Menschen mit Behinderung darin unterstützen, Arbeit auf } \\
\text { dem ersten Arbeitsmarkt zu finden. }\end{array}$ \\
\hline
\end{tabular}




\begin{tabular}{|c|c|c|c|c|c|c|c|c|c|c|c|c|c|c|}
\hline \multirow{2}{*}{\multicolumn{3}{|c|}{ Itembezeichnung }} & \multicolumn{12}{|c|}{ Itemkennwerte } \\
\hline & & & \multicolumn{4}{|c|}{ MZP1 } & \multicolumn{4}{|c|}{ MZP2 } & \multicolumn{4}{|c|}{ MZP3 } \\
\hline MZP1 & MZP2 & MZP3 & MW & SD & $\mathrm{r}_{\mathrm{it}}$ & $\lambda_{\mathrm{ij}}$ & MW & SD & $\mathrm{r}_{\mathrm{it}}$ & $\lambda_{\mathrm{ij}}$ & MW & SD & $\mathrm{r}_{\mathrm{it}}$ & $\lambda_{\mathrm{ij}}$ \\
\hline a_brk14 & b_brk14 & c_brk14 & 5.03 & 1.10 & .50 & $.56^{* *}$ & 5.07 & 1.08 & .61 & $.71 * *$ & 4.88 & 1.18 & .65 & $.72 * *$ \\
\hline a_brk15 & b_brk15 & c_brk15 & 5.09 & 1.06 & .53 & $.59 * *$ & 5.02 & 1.08 & .59 & $.67 * *$ & 4.81 & 1.24 & .61 & $.65^{* *}$ \\
\hline a_brk16 & b_brk16 & c_brk16 & 5.04 & 1.04 & .48 & $.61 * *$ & 5.06 & 1.09 & .64 & $.80^{* *}$ & 4.92 & 1.16 & .62 & $.77^{* *}$ \\
\hline a_brk18 & b_brk18 & c_brk18 & 4.56 & 1.45 & .44 & $.55^{* *}$ & 4.47 & 1.43 & .43 & $.47 * *$ & 4.14 & 1.46 & .42 & $.46^{* *}$ \\
\hline a_brk21 & b_brk21 & c_brk21 & 4.97 & 1.18 & .47 & $.58^{* *}$ & 4.98 & 1.14 & .51 & $.58^{* *}$ & 4.85 & 1.25 & .48 & $.55^{* *}$ \\
\hline \multirow{4}{*}{\multicolumn{3}{|c|}{ Skalenkennwerte }} & \multicolumn{2}{|c|}{ Cronbachs $\alpha$} & .73 & & \multicolumn{2}{|c|}{ Cronbachs $\alpha$} & .79 & & \multicolumn{2}{|c|}{ Cronbachs $\alpha$} & \multicolumn{2}{|l|}{.79} \\
\hline & & & \multicolumn{2}{|l|}{ MW } & 4.93 & & \multicolumn{2}{|c|}{ MW } & \multicolumn{2}{|l|}{4.92} & \multicolumn{2}{|l|}{ MW } & \multicolumn{2}{|l|}{4.72} \\
\hline & & & \multicolumn{2}{|l|}{ SD } & \multicolumn{2}{|l|}{.82} & \multicolumn{2}{|l|}{ SD } & \multicolumn{2}{|l|}{.86} & \multicolumn{2}{|l|}{ SD } & \multicolumn{2}{|l|}{.91} \\
\hline & & & \multicolumn{2}{|l|}{$\mathrm{N}$} & \multicolumn{2}{|l|}{436} & \multicolumn{2}{|l|}{$\mathrm{N}$} & \multicolumn{2}{|l|}{399} & \multicolumn{2}{|l|}{$\mathrm{N}$} & \multicolumn{2}{|l|}{337} \\
\hline & & & \multicolumn{2}{|c|}{$\chi^{2}[\mathrm{df}]$} & \multicolumn{2}{|c|}{5.64 n.s. [4] } & \multicolumn{2}{|c|}{$\chi^{2}[\mathrm{df}]$} & \multicolumn{2}{|c|}{6.67 n.s. [4] } & \multicolumn{2}{|c|}{$\chi^{2}[\mathrm{df}]$} & \multicolumn{2}{|c|}{8.16 n.s. [4] } \\
\hline & & & CFI & & .99 & & CFI & & 1.00 & & CFI & & .99 & \\
\hline Messmoc & & & TLI & & .97 & & TLI & & .99 & & TLI & & .98 & \\
\hline & & & RMSF & & $.05 \mathrm{n}$ & & RMS & & $.04 \mathrm{n}$ & & RMS & & $.06 \mathrm{n}$ & \\
\hline & & & SRMF & & .02 & & SRM & & .02 & & SRM & & .02 & \\
\hline
\end{tabular}

$* * \mathrm{p} \leq .01 \quad * \mathrm{p} \leq .05 \quad$ n.s. $=$ nicht signifikant 


\subsubsection{Barrierefreiheit}

Kurzbezeichnung Variablenname

Instruktion

Quelle

Weiterführende Literatur

Skalierung (Antwortformat)

Inverse Items (-)

Anzahl Items

Skalenbildung

Instrument xa_brk_b/xb_brk_b/xc_brk_b

(xa_brk_Bar/xb_brk_Bar/xc_brk_Bar)

Wie sehr stimmst du folgenden Aussagen zu?

Eigenentwicklung basierend auf der UN-

Behindertenrechtskonvention (2009)

Degener \& Diehl, 2015

Endpolbeschriftung; 1 = stimme überhaupt nicht zu, $(\ldots), 6=$ stimme voll $\mathrm{zu}$

5

per Mittelwert

Hauptfragebogen Messzeitpunkt 1, 2 und 3

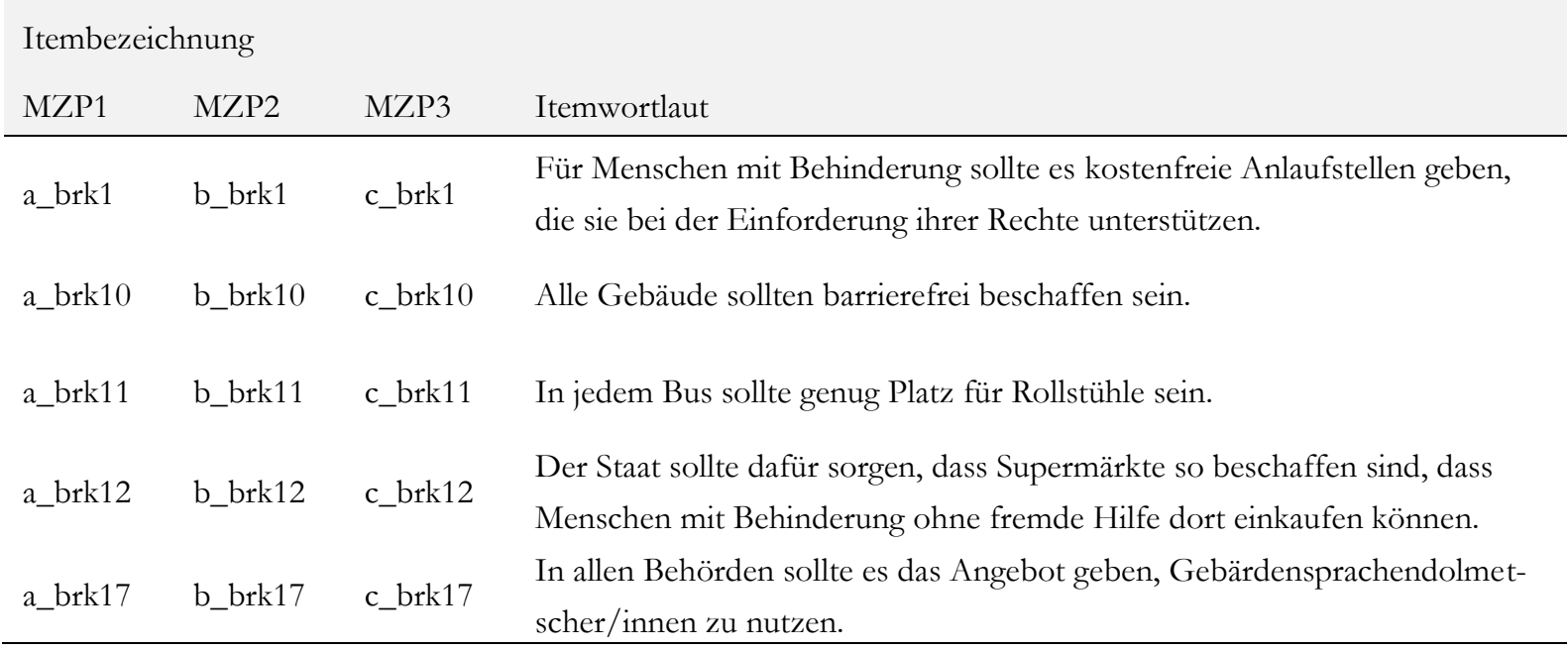




\begin{tabular}{|c|c|c|c|c|c|c|c|c|c|c|c|c|c|c|}
\hline \multirow{2}{*}{\multicolumn{3}{|c|}{ Itembezeichnung }} & \multicolumn{12}{|c|}{ Itemkennwerte } \\
\hline & & & \multicolumn{4}{|c|}{ MZP1 } & \multicolumn{4}{|c|}{ MZP2 } & \multicolumn{4}{|c|}{ MZP3 } \\
\hline MZP1 & MZP2 & MZP3 & MW & SD & $r_{i t}$ & $\lambda_{\mathrm{ij}}$ & MW & SD & $\mathrm{r}_{\mathrm{it}}$ & $\lambda_{\mathrm{ij}}$ & MW & SD & $\mathrm{r}_{\mathrm{it}}$ & $\lambda_{\mathrm{ij}}$ \\
\hline a_brk1 & b_brk1 & c_brk1 & 5.13 & 1.09 & .36 & $.41 * *$ & 5.08 & 1.11 & .36 & $.39 * *$ & 5.06 & 1.23 & .55 & $.58^{* *}$ \\
\hline a_brk10 & b_brk10 & c_brk10 & 4.95 & 1.27 & .44 & $.54^{* *}$ & 5.21 & 1.15 & .57 & $.71 * *$ & 5.19 & 1.17 & .65 & $.73^{* *}$ \\
\hline a_brk11 & b_brk11 & c_brk11 & 5.67 & .76 & .57 & $.72 * *$ & 5.60 & .78 & .68 & $.81 * *$ & 5.54 & .90 & .73 & $.84^{* *}$ \\
\hline a_brk12 & b_brk12 & c_brk12 & 5.35 & 1.05 & .55 & $.72 * *$ & 5.45 & .91 & .57 & $.72^{* *}$ & 5.31 & 1.14 & .63 & $.73^{* *}$ \\
\hline a_brk17 & b_brk17 & c_brk17 & 5.14 & 1.08 & .50 & $.56^{* *}$ & 5.18 & 1.13 & .52 & $.55^{* *}$ & 5.17 & 1.09 & .63 & $.69^{* *}$ \\
\hline \multirow{4}{*}{\multicolumn{3}{|c|}{ Skalenkennwerte }} & \multicolumn{2}{|c|}{ Cronbachs $\alpha$} & \multicolumn{2}{|l|}{.73} & \multicolumn{2}{|c|}{ Cronbachs $\alpha$} & \multicolumn{2}{|l|}{.78} & \multicolumn{2}{|c|}{ Cronbachs $\alpha$} & \multicolumn{2}{|l|}{.84} \\
\hline & & & \multicolumn{2}{|l|}{ MW } & \multicolumn{2}{|l|}{5.23} & \multicolumn{2}{|l|}{ MW } & \multicolumn{2}{|l|}{5.29} & \multicolumn{2}{|l|}{ MW } & \multicolumn{2}{|l|}{5.25} \\
\hline & & & \multicolumn{2}{|l|}{ SD } & \multicolumn{2}{|l|}{.74} & \multicolumn{2}{|l|}{ SD } & \multicolumn{2}{|l|}{.76} & \multicolumn{2}{|l|}{ SD } & \multicolumn{2}{|l|}{.86} \\
\hline & & & \multicolumn{2}{|l|}{$\mathrm{N}$} & \multicolumn{2}{|l|}{441} & \multicolumn{2}{|l|}{$\mathrm{N}$} & \multicolumn{2}{|l|}{392} & \multicolumn{2}{|l|}{$\mathrm{N}$} & \multicolumn{2}{|l|}{332} \\
\hline & & & \multicolumn{2}{|c|}{$\chi^{2}[\mathrm{df}]$} & \multicolumn{2}{|c|}{10.56 n.s. [5] } & \multicolumn{2}{|c|}{$\chi^{2}[\mathrm{df}]$} & 13.81 & & $\chi^{2}[\mathrm{~d}$ & & 25.09 & \\
\hline & & & CFI & & .99 & & CFI & & .98 & & CFI & & .97 & \\
\hline Messmoc & & & TLI & & .97 & & TLI & & .97 & & TLI & & .94 & \\
\hline & & & RMSF & & $.05 \mathrm{n}$ & & RMS & & $.07 \mathrm{n}$ & & RMS & & $.11^{* *}$ & \\
\hline & & & SRMF & & .02 & & SRM & & .03 & & SRM & & .03 & \\
\hline
\end{tabular}

$* * \mathrm{p} \leq .01 \quad * \mathrm{p} \leq .05 \quad$ n.s. $=$ nicht signifikant 


\subsubsection{Politische Rechte}

Kurzbezeichnung Variablenname

Instruktion

Quelle

Weiterführende Literatur

Skalierung (Antwortformat)

Inverse Items (-)

Anzahl Items

Skalenbildung

Instrument xa_brk_p/xb_brk_p/xc_brk_p

(xa_brk_Pol/xb_brk_Pol/xc_brk_Pol)

Wie sehr stimmst du folgenden Aussagen zu?

Eigenentwicklung basierend auf der UN-

Behindertenrechtskonvention (2009)

Degener \& Diehl, 2015

Endpolbeschriftung; 1 = stimme überhaupt nicht zu, $(\ldots), 6=$ stimme voll $\mathrm{zu}$

4

per Mittelwert

Hauptfragebogen Messzeitpunkt 1, 2 und 3

\begin{tabular}{|c|c|c|c|}
\hline MZP1 & MZP2 & MZP3 & Itemwortlaut \\
\hline a_brk5 & b_brk5 & c_brk5 & $\begin{array}{l}\text { Menschen mit Behinderung sollten die gleichen politischen Rechte haben } \\
\text { (z. B. aktives Wahlrecht) wie Menschen ohne Behinderung. }\end{array}$ \\
\hline a_brk6 & b_brk6 & c_brk6 & $\begin{array}{l}\text { Menschen mit Behinderung sollten bei öffentlichen Angelegenheiten das } \\
\text { gleiche Mitspracherecht haben wie Menschen ohne Behinderung. }\end{array}$ \\
\hline a_brk7 & b_brk7 & c_brk7 & $\begin{array}{l}\text { Menschen mit Behinderung sollten darin unterstützt werden, sich politisch } \\
\text { zu engagieren. }\end{array}$ \\
\hline a_brk9 & b_brk9 & c_brk9 & $\begin{array}{l}\text { Menschen mit Behinderung sollten bei Gesetzesänderungen, die ihre } \\
\text { Gleichberechtigung mit anderen betreffen, mitentscheiden dürfen. }\end{array}$ \\
\hline
\end{tabular}




\begin{tabular}{|c|c|c|c|c|c|c|c|c|c|c|c|c|c|c|}
\hline \multirow{2}{*}{\multicolumn{3}{|c|}{ Itembezeichnung }} & \multicolumn{12}{|c|}{ Itemkennwerte } \\
\hline & & & \multicolumn{4}{|c|}{ MZP1 } & \multicolumn{4}{|c|}{ MZP2 } & \multicolumn{4}{|c|}{ MZP3 } \\
\hline MZP1 & MZP2 & MZP3 & MW & $\mathrm{SD}$ & $\mathrm{r}_{\mathrm{it}}$ & $\lambda_{\mathrm{ij}}$ & MW & SD & $r_{i t}$ & $\lambda_{\mathrm{ij}}$ & MW & SD & $\mathrm{r}_{\mathrm{it}}$ & $\lambda_{\mathrm{ij}}$ \\
\hline a_brk5 & b_brk5 & c_brk5 & 4.81 & 1.36 & .73 & $.72^{* *}$ & 4.72 & 1.34 & .69 & $.77 * *$ & 4.59 & 1.35 & .71 & $.67 * *$ \\
\hline a_brk6 & b_brk6 & c_brk6 & 5.10 & 1.14 & .78 & $.76^{* *}$ & 4.86 & 1.27 & .79 & $.90^{* *}$ & 4.81 & 1.20 & .80 & $.82 * *$ \\
\hline a_brk7 & b_brk7 & c_brk7 & 5.03 & 1.13 & .70 & $.83^{* *}$ & 4.89 & 1.14 & .75 & $.81 * *$ & 4.89 & 1.12 & .72 & $.85^{* *}$ \\
\hline a_brk9 & b_brk9 & c_brk9 & 5.19 & 1.06 & .58 & $.67 * *$ & 5.10 & 1.10 & .63 & $.68^{* *}$ & 5.00 & 1.15 & .66 & $.75^{* *}$ \\
\hline \multirow{4}{*}{\multicolumn{3}{|c|}{ Skalenkennwerte }} & \multicolumn{2}{|c|}{ Cronbachs $\alpha$} & \multicolumn{2}{|l|}{.85} & \multicolumn{2}{|c|}{ Cronbachs $\alpha$} & \multicolumn{2}{|l|}{.87} & \multicolumn{2}{|c|}{ Cronbachs $\alpha$} & \multicolumn{2}{|l|}{.87} \\
\hline & & & \multicolumn{2}{|l|}{ MW } & \multicolumn{2}{|l|}{5.03} & \multicolumn{2}{|l|}{ MW } & \multicolumn{2}{|l|}{4.88} & \multicolumn{2}{|l|}{ MW } & \multicolumn{2}{|c|}{4.80} \\
\hline & & & \multicolumn{2}{|l|}{ SD } & \multicolumn{2}{|l|}{.98} & \multicolumn{2}{|l|}{ SD } & \multicolumn{2}{|l|}{1.03} & \multicolumn{2}{|l|}{$\mathrm{SD}$} & \multicolumn{2}{|l|}{1.05} \\
\hline & & & \multicolumn{2}{|l|}{$\mathrm{N}$} & \multicolumn{2}{|l|}{436} & \multicolumn{2}{|l|}{$\mathrm{N}$} & \multicolumn{2}{|l|}{405} & \multicolumn{2}{|l|}{$\mathrm{N}$} & \multicolumn{2}{|l|}{339} \\
\hline & & & \multicolumn{2}{|c|}{$\chi^{2}[\mathrm{df}]$} & \multicolumn{2}{|l|}{$3.88^{*}[1]$} & \multicolumn{2}{|c|}{$\chi^{2}[\mathrm{df}]$} & \multicolumn{2}{|l|}{.43 n.s. [1] } & $\chi^{2}[\mathrm{df}]$ & & 2.66 & \\
\hline & & & CFI & & 1.00 & & CFI & & 1.00 & & CFI & & 1.00 & \\
\hline Messme & & & TLI & & .98 & & TLI & & 1.00 & & TLI & & .99 & \\
\hline & & & RMS & & .08 n.s. & & RMSI & & .00 n.s. & & RMSI & & $.07 \mathrm{n}$ & \\
\hline & & & SRM & & .01 & & SRMI & & .00 & & SRMI & & .01 & \\
\hline
\end{tabular}

** $\mathrm{p} \leq .01 \quad * \mathrm{p} \leq .05 \quad$ n.s. $=$ nicht signifikant 


\subsubsection{Strukturmodell Befürwortung von Inklusion}

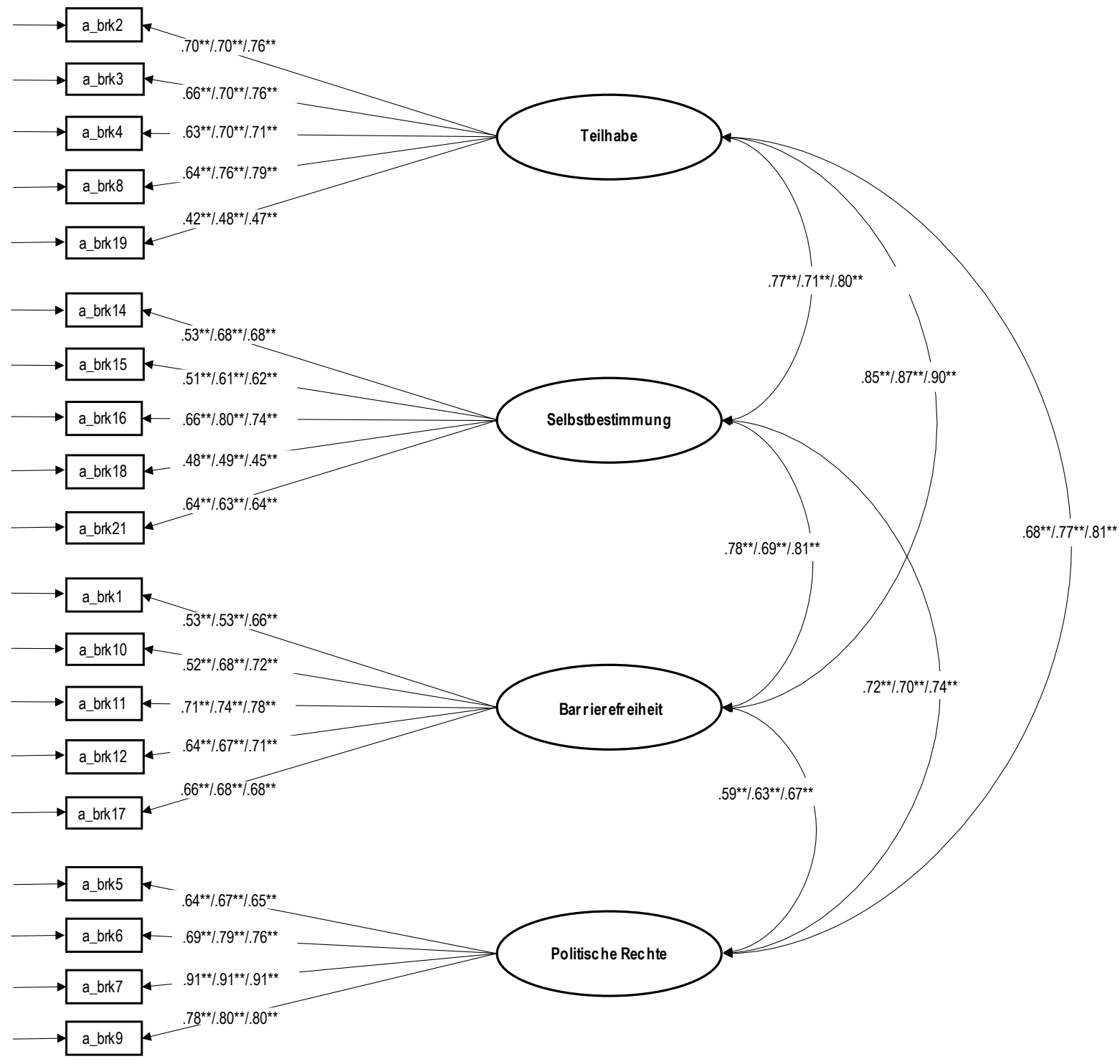

${ }^{* *} \mathrm{p} \leq .01 \quad * \mathrm{p} \leq .05 \quad$ n.s. $=$ nicht signifikant

Modellfitwerte (MZP1/MZP2/MZP3):

$\chi^{2}[\mathrm{df}]=371.07 * *[142] / 514.01 * *[142] / 398.28 * *[142] ; \mathrm{CFI}=.89 / .89 / .92 ; \mathrm{TLI}=.91 / .87 / .91 ; \mathrm{RMSE}-$

$\mathrm{A}=.06 * / .08 * / .07 * ; \mathrm{SRMR}=.05 / .06 / .05$ 



\subsection{Befürwortung von schulischer Inklusion}

\subsubsection{Schulische Inklusion von Schüler*innen mit körperlicher Behinderung}

\subsubsection{Vorteile von Inklusion für Schüler*innen mit körperlicher Bebinderung}

Kurzbezeichnung Variablenname

Instruktion

Quelle

Weiterführende Literatur

Skalierung (Antwortformat)

Inverse Items (-)

Anzahl Items

Skalenbildung

Instrument xa_kbesb/xb_kbesb/xb_kbesb (xa_ikb_EffB/xb_ikb_EffB/xc_ikb_EffB)

Als nächstes würden wir gern etwas über darüber erfahren, wie du es einschätzt, wenn Kinder mit bestimmten Behinderungsformen oder Erkrankungen auf Regelschulen gehen.

Zuerst geht es um deine Einschätzungen dazu, wenn Kinder mit körperlicher Behinderung auf Regelschulen gehen.

Wie sehr stimmst du folgenden Aussagen zu?

Gebhardt et al., 2011; Kunz, Luder \& Moretti, 2010; Paulus, 2013

Werning, 2014; Sturm \& Wagner-Willi, 2018

Endpolbeschriftung; 1 = stimme überhaupt nicht zu, (...), 6 = stimme voll $\mathrm{zu}$

a_ikb7, b_ikb7, c_ikb7

4

per Mittelwert

Hauptfragebogen Messzeitpunkt 1, 2 und 3

Itembezeichnung

\begin{tabular}{|c|c|c|c|}
\hline MZP1 & MZP2 & MZP3 & Itemwortlaut \\
\hline a_ikb7 & b_ikb7 & c_ikb7 & $\begin{array}{l}\text { Kinder mit körperlicher Behinderung würden in Förderschulen mehr ler- } \\
\text { nen. (-) }\end{array}$ \\
\hline a_ikb10 & b_ikb10 & c_ikb10 & $\begin{array}{l}\text { Kinder mit körperlicher Behinderung können in Regelschulen besser zu } \\
\text { selbstständigem Lernen angeregt werden als in Förderschulen. }\end{array}$ \\
\hline a_ikb12 & b_ikb12 & c_ikb12 & $\begin{array}{l}\text { Kinder mit körperlicher Behinderung entwickeln in Regelschulen ein größe- } \\
\text { res Selbstbewusstsein als in Förderschulen. }\end{array}$ \\
\hline a_ikb15 & b_ikb15 & c_ikb15 & $\begin{array}{l}\text { Kinder mit körperlicher Behinderung lernen in Regelschulen wichtige Din- } \\
\text { ge, die sie in Förderschulen nicht lernen würden. }\end{array}$ \\
\hline
\end{tabular}

(-) inverse Items 


\begin{tabular}{|c|c|c|c|c|c|c|c|c|c|c|c|c|c|c|}
\hline \multirow{2}{*}{\multicolumn{3}{|c|}{ Itembezeichnung }} & \multicolumn{12}{|c|}{ Itemkennwerte } \\
\hline & & & \multicolumn{4}{|c|}{ MZP1 } & \multicolumn{4}{|c|}{ MZP2 } & \multicolumn{4}{|c|}{ MZP3 } \\
\hline MZP1 & MZP2 & MZP3 & MW & SD & $\mathrm{r}_{\mathrm{it}}$ & $\lambda_{\mathrm{ij}}$ & MW & SD & $\mathrm{r}_{\mathrm{it}}$ & $\lambda_{\mathrm{ij}}$ & MW & $\mathrm{SD}$ & $\mathrm{r}_{\mathrm{it}}$ & $\lambda_{\mathrm{ij}}$ \\
\hline ra_ikb7 & rb_ikb7 & rc_ikb7 & 4.25 & 1.44 & .40 & $.47 * *$ & 4.31 & 1.46 & .48 & $.57 * *$ & 4.37 & 1.41 & .46 & $.56^{* *}$ \\
\hline a_ikb10 & b_ikb10 & c_ikb10 & 4.10 & 1.36 & .59 & $.73^{* *}$ & 4.13 & 1.36 & .70 & $.88^{* *}$ & 4.31 & 1.24 & .66 & $.82^{* *}$ \\
\hline a_ikb12 & b_ikb12 & c_ikb12 & 3.91 & 1.35 & .50 & $.63 * *$ & 4.03 & 1.30 & .51 & $.56^{* *}$ & 4.02 & 1.31 & .46 & $.55^{* *}$ \\
\hline a_ikb15 & b_ikb15 & c_ikb15 & 4.31 & 1.40 & .59 & $.71 * *$ & 4.44 & 1.35 & .56 & $.66^{* *}$ & 4.41 & 1.37 & .62 & $.76^{* *}$ \\
\hline \multirow{4}{*}{\multicolumn{3}{|c|}{ Skalenkennwerte }} & \multicolumn{2}{|c|}{ Cronbachs $\alpha$} & \multicolumn{2}{|l|}{.73} & \multicolumn{2}{|c|}{ Cronbachs $\alpha$} & \multicolumn{2}{|l|}{.77} & \multicolumn{2}{|c|}{ Cronbachs $\alpha$} & \multicolumn{2}{|l|}{.76} \\
\hline & & & \multicolumn{2}{|l|}{ MW } & \multicolumn{2}{|l|}{4.15} & \multicolumn{2}{|l|}{ MW } & \multicolumn{2}{|c|}{4.23} & \multicolumn{2}{|l|}{ MW } & \multicolumn{2}{|c|}{4.26} \\
\hline & & & \multicolumn{2}{|l|}{ SD } & \multicolumn{2}{|l|}{1.03} & \multicolumn{2}{|l|}{ SD } & \multicolumn{2}{|c|}{1.04} & \multicolumn{2}{|l|}{ SD } & \multicolumn{2}{|l|}{1.03} \\
\hline & & & \multicolumn{2}{|l|}{$\mathrm{N}$} & \multicolumn{2}{|l|}{433} & \multicolumn{2}{|l|}{$\mathrm{N}$} & \multicolumn{2}{|l|}{394} & \multicolumn{2}{|l|}{$\mathrm{N}$} & \multicolumn{2}{|l|}{337} \\
\hline & & & \multicolumn{2}{|c|}{$\chi^{2}[\mathrm{df}]$} & \multicolumn{2}{|c|}{5.18 n.s. [2] } & \multicolumn{2}{|c|}{$\chi^{2}[\mathrm{df}]$} & \multicolumn{2}{|c|}{$12.45^{* *}[2]$} & $\chi^{2}[\mathrm{df}$ & & 1.26 & \\
\hline & & & CFI & & .99 & & CFI & & .97 & & CFI & & 1.00 & \\
\hline Messmo & & & TLI & & .97 & & TLI & & .92 & & TLI & & 1.01 & \\
\hline & & & RMSI & & $.06 \mathrm{n}$ & & RMS & & $.12^{*}$ & & RMSI & & 0.00 & \\
\hline & & & SRMH & & .02 & & SRM & & .03 & & SRMI & & .01 & \\
\hline
\end{tabular}

** $\mathrm{p} \leq .01 \quad * \mathrm{p} \leq .05 \quad$ n.s. $=$ nicht signifikant 


\subsubsection{Vorteile von Inklusion für Mitschüler*innen}

Kurzbezeichnung Variablenname

Instruktion

Quelle

Weiterführende Literatur

Skalierung (Antwortformat)

Inverse Items (-)

Anzahl Items

Skalenbildung

Instrument xa_kbems/xb_kbems/xc_kbems

(xa_ikb_EffD/xb_ikb_EffD/xc_ikb_EffD)

Wie sehr stimmst du folgenden Aussagen zu?

Bosse \& Spörer, 2014; Gebhardt et al., 2011; Kunz et al., 2010; Paulus, 2013

Werning, 2014; Sturm \& Wagner-Willi, 2018

Endpolbeschriftung; 1 = stimme überhaupt nicht $\mathrm{zu}$, (...), 6 = stimme voll zu

a_ikb11, b_ikb11, c_ikb11

4

per Mittelwert

Hauptfragebogen Messzeitpunkt 1, 2 und 3

\begin{tabular}{|c|c|c|c|}
\hline \multicolumn{3}{|c|}{ Itembezeichnung } & \multirow[b]{2}{*}{ Itemwortlaut } \\
\hline MZP1 & MZP2 & MZP3 & \\
\hline a_ikb8 & b_ikb8 & c_ikb8 & $\begin{array}{l}\text { Durch Kinder mit körperlicher Behinderung lernen die anderen Kinder } \\
\text { verstärkt soziale Fähigkeiten (z. B. einander helfen). }\end{array}$ \\
\hline a_ikb11 & b_ikb11 & c_ikb11 & $\begin{array}{l}\text { Die anderen Kinder haben einen Nachteil, weil Kinder mit körperlicher } \\
\text { Behinderung alle Aufmerksamkeit der Lehrkräfte brauchen. (-) }\end{array}$ \\
\hline a_ikb13 & b_ikb13 & c_ikb13 & $\begin{array}{l}\text { Die Anwesenheit von Kindern mit körperlicher Behinderung fördert bei } \\
\text { den anderen Kindern die Akzeptanz von Unterschiedlichkeit. }\end{array}$ \\
\hline a_ikb14 & b_ikb14 & c_ikb14 & $\begin{array}{l}\text { Wenn Kinder mit körperlicher Behinderung in der Klasse sind, hat das } \\
\text { auch für die anderen Kinder Vorteile, trotz möglicher Schwierigkeiten. }\end{array}$ \\
\hline
\end{tabular}

(-) inverse Items 


\begin{tabular}{|c|c|c|c|c|c|c|c|c|c|c|c|c|c|c|}
\hline \multirow{2}{*}{\multicolumn{3}{|c|}{ Itembezeichnung }} & \multicolumn{12}{|c|}{ Itemkennwerte } \\
\hline & & & \multicolumn{4}{|c|}{ MZP1 } & \multicolumn{4}{|c|}{ MZP2 } & \multicolumn{4}{|c|}{ MZP3 } \\
\hline MZP1 & MZP2 & MZP3 & MW & SD & $\mathrm{r}_{\mathrm{it}}$ & $\lambda_{\mathrm{ij}}$ & MW & SD & $r_{i t}$ & $\lambda_{\mathrm{ij}}$ & MW & $\mathrm{SD}$ & $\mathrm{r}_{\mathrm{it}}$ & $\lambda_{\mathrm{ij}}$ \\
\hline a_ikb8 & b_ikb8 & c_ikb8 & 5.35 & .96 & .52 & $.63^{* *}$ & 5.25 & 1.05 & .58 & $.71 * *$ & 5.10 & 1.15 & .53 & $.64 * *$ \\
\hline ra_ikb11 & rb_ikb11 & rc_ikb11 & 4.42 & 1.36 & .36 & $.39 * *$ & 4.54 & 1.25 & .36 & $.41 * *$ & 4.52 & 1.35 & .32 & $.37^{* *}$ \\
\hline a_ikb13 & b_ikb13 & c_ikb13 & 5.17 & 1.08 & .62 & $.82^{* *}$ & 5.05 & 1.19 & .61 & $.79 * *$ & 4.97 & 1.16 & .57 & $.75^{* *}$ \\
\hline a_ikb14 & b_ikb14 & c_ikb14 & 4.81 & 1.19 & .64 & $.76^{* *}$ & 4.73 & 1.23 & .55 & $.67 * *$ & 4.73 & 1.21 & .57 & $.74 * *$ \\
\hline \multirow{4}{*}{\multicolumn{3}{|c|}{ Skalenkennwerte }} & \multicolumn{2}{|c|}{ Cronbachs $\alpha$} & \multicolumn{2}{|l|}{.75} & \multicolumn{2}{|c|}{ Cronbachs $\alpha$} & \multicolumn{2}{|l|}{.74} & \multicolumn{2}{|c|}{ Cronbachs $\alpha$} & \multicolumn{2}{|l|}{.72} \\
\hline & & & \multicolumn{2}{|l|}{ MW } & \multicolumn{2}{|l|}{4.93} & \multicolumn{2}{|l|}{ MW } & \multicolumn{2}{|c|}{4.90} & \multicolumn{2}{|l|}{ MW } & \multicolumn{2}{|c|}{4.82} \\
\hline & & & \multicolumn{2}{|l|}{ SD } & \multicolumn{2}{|l|}{.86} & \multicolumn{2}{|l|}{$\mathrm{SD}$} & \multicolumn{2}{|l|}{.88} & \multicolumn{2}{|l|}{$\mathrm{SD}$} & \multicolumn{2}{|l|}{.89} \\
\hline & & & \multicolumn{2}{|l|}{$\mathrm{N}$} & \multicolumn{2}{|l|}{435} & \multicolumn{2}{|l|}{$\mathrm{N}$} & \multicolumn{2}{|l|}{395} & \multicolumn{2}{|l|}{$\mathrm{N}$} & \multicolumn{2}{|l|}{336} \\
\hline & & & \multicolumn{2}{|c|}{$\chi^{2}[\mathrm{df}]$} & \multicolumn{2}{|l|}{$7.38^{*}[2]$} & \multicolumn{2}{|c|}{$\chi^{2}[\mathrm{df}]$} & \multicolumn{2}{|c|}{1.10 n.s. [2] } & $\chi^{2}[\mathrm{~d}$ & & 1.48 & \\
\hline & & & CFI & & .99 & & CFI & & 1.00 & & CFI & & 1.00 & \\
\hline Messmoc & & & TLI & & .96 & & TLI & & 1.01 & & TLI & & 1.01 & \\
\hline & & & RMSI & & .08 n.s. & & RMS & & $.00 \mathrm{t}$ & & RMS & & $.00 \mathrm{t}$ & \\
\hline & & & SRMI & & .02 & & SRM & & .01 & & SRM & & .01 & \\
\hline
\end{tabular}

** $\mathrm{p} \leq .01 \quad * \mathrm{p} \leq .05 \quad$ n.s. $=$ nicht signifikant 


\subsubsection{Strukturmodell Einstellungen zu schulischer Inklusion bei körperlicher Behinderung}

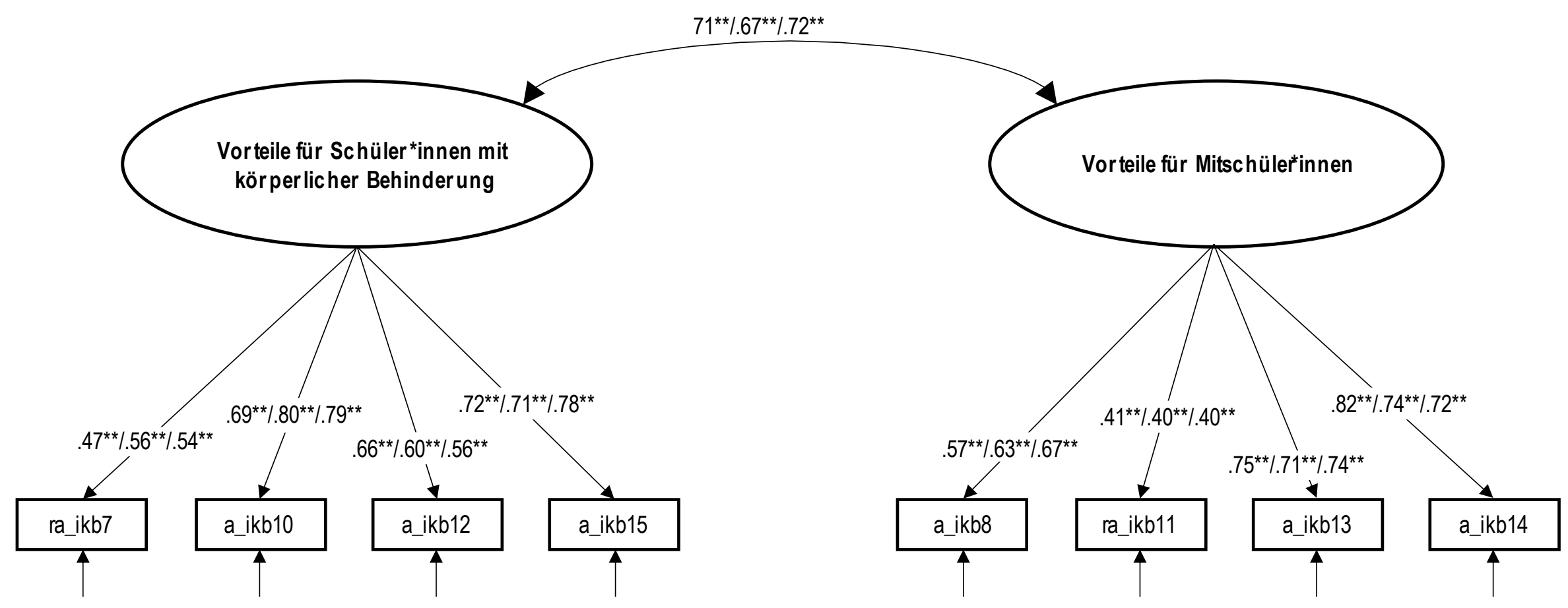

** $\mathrm{p} \leq .01 \quad * \mathrm{p} \leq .05 \quad$ n.s. $=$ nicht signifikant

Modellfitwerte (MZP1/MZP2/MZP3):

$\chi^{2}[\mathrm{df}]=28.61^{*}[17] / 45.30^{* *}[17] / 29.96^{*}[17] ; \mathrm{CFI}=.99 / .97 / .98 ; \mathrm{TLI}=.98 / .95 / .97 ; \mathrm{RMSEA}=.04$ n.s. $/ .07$ n.s. $/ .05$ n.s.; SRMR $=.03 / .03 / .03$ 



\subsubsection{Schulische Inklusion von Schüler*innen mit geistiger Behinderung}

\subsubsection{Vorteile von Inklusion für Schüler*innen mit geistiger Behinderung}

Kurzbezeichnung Variablenname

Instruktion

Quelle

Weiterführende Literatur

Skalierung (Antwortformat)

Inverse Items (-)

Anzahl Items

Skalenbildung

Instrument xa_gbesb/xb_gbesb/xc_gbesb

(xa_igb_EffB/xb_igb_EffB/xc_igb_EffB)

Nun geht es um Einschätzungen dazu, wenn Kinder mit geistiger Behinderung auf Regelschulen gehen.

Wie sehr stimmst du folgenden Aussagen zu?

Gebhardt et al., 2011; Kunz et al., 2010; Paulus, 2013

Werning, 2014; Sturm \& Wagner-Willi, 2018

Endpolbeschriftung; 1 = stimme überhaupt nicht zu, (...), 6 = stimme voll $\mathrm{zu}$

a_igb7, b_igb7, c_igb7

4

per Mittelwert

Hauptfragebogen Messzeitpunkt 1, 2 und 3

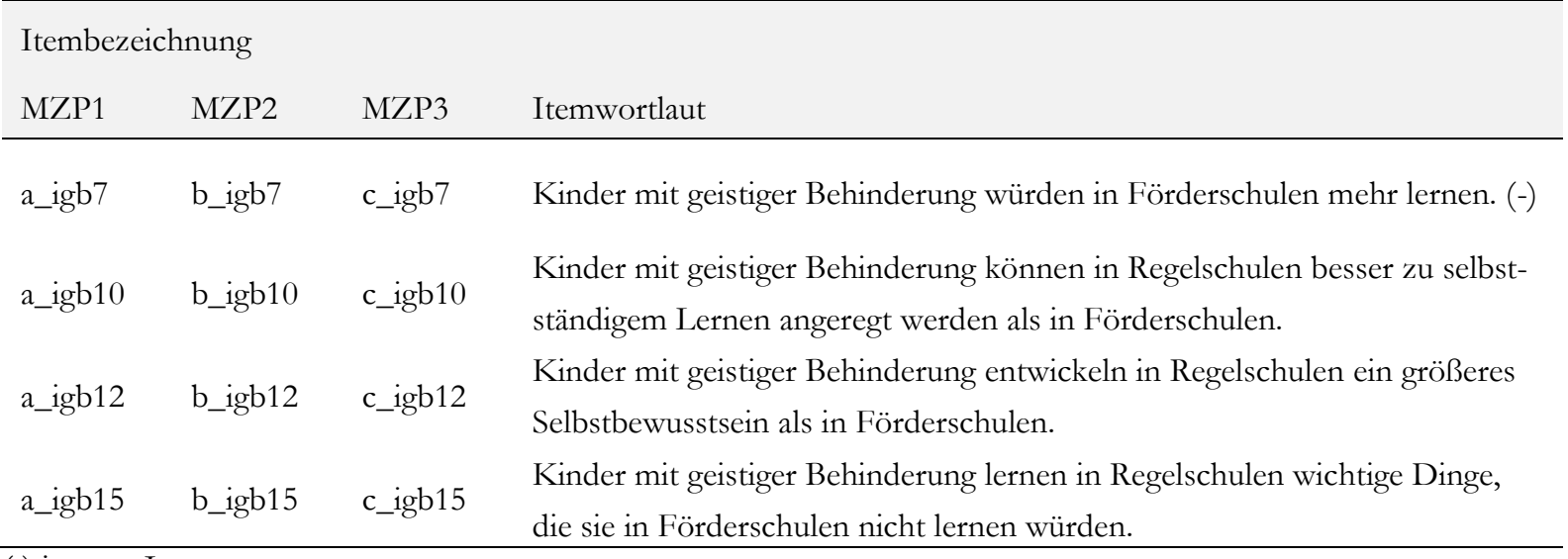

(-) inverse Items 


\begin{tabular}{|c|c|c|c|c|c|c|c|c|c|c|c|c|c|c|}
\hline \multirow{2}{*}{\multicolumn{3}{|c|}{ Itembezeichnung }} & \multicolumn{12}{|c|}{ Itemkennwerte } \\
\hline & & & \multicolumn{4}{|c|}{ MZP1 } & \multicolumn{4}{|c|}{ MZP2 } & \multicolumn{4}{|c|}{ MZP3 } \\
\hline MZP1 & MZP2 & MZP3 & MW & SD & $\mathrm{r}_{\mathrm{it}}$ & $\lambda_{\mathrm{ij}}$ & MW & SD & $\mathrm{r}_{\mathrm{it}}$ & $\lambda_{\mathrm{ij}}$ & MW & SD & $\mathrm{r}_{\mathrm{it}}$ & $\lambda_{\mathrm{ij}}$ \\
\hline ra_igb7 & rb_igb7 & rc_igb7 & 2.72 & 1.41 & .45 & $.50^{* *}$ & 2.88 & 1.41 & .33 & $.38^{* *}$ & 2.70 & 1.45 & .49 & $.54 * *$ \\
\hline a_igb10 & b_igb10 & c_igb10 & 3.13 & 1.37 & .65 & $.75^{* *}$ & 3.23 & 1.37 & .66 & $.78^{* *}$ & 3.12 & 1.37 & .68 & $.79 * *$ \\
\hline a_igb12 & b_igb12 & c_igb12 & 3.24 & 1.35 & .65 & $.78^{* *}$ & 3.43 & 1.34 & .61 & $.73^{* *}$ & 3.31 & 1.35 & .68 & $.80^{* *}$ \\
\hline a_igb15 & b_igb15 & c_igb15 & 3.54 & 1.43 & .59 & $.70^{* *}$ & 3.63 & 1.40 & .61 & $.75^{* *}$ & 3.54 & 1.44 & .59 & $.69 * *$ \\
\hline \multirow{4}{*}{\multicolumn{3}{|c|}{ Skalenkennwerte }} & \multicolumn{2}{|c|}{ Cronbachs $\alpha$} & \multicolumn{2}{|l|}{.78} & \multicolumn{2}{|c|}{ Cronbachs $\alpha$} & \multicolumn{2}{|l|}{.76} & \multicolumn{2}{|c|}{ Cronbachs $\alpha$} & \multicolumn{2}{|l|}{.80} \\
\hline & & & \multicolumn{2}{|l|}{ MW } & \multicolumn{2}{|l|}{3.16} & \multicolumn{2}{|l|}{ MW } & \multicolumn{2}{|l|}{3.32} & \multicolumn{2}{|l|}{ MW } & \multicolumn{2}{|l|}{3.21} \\
\hline & & & \multicolumn{2}{|l|}{$\mathrm{SD}$} & \multicolumn{2}{|l|}{1.08} & \multicolumn{2}{|l|}{$\mathrm{SD}$} & \multicolumn{2}{|l|}{1.06} & \multicolumn{2}{|l|}{$\mathrm{SD}$} & \multicolumn{2}{|l|}{1.11} \\
\hline & & & \multicolumn{2}{|l|}{$\mathrm{N}$} & \multicolumn{2}{|l|}{428} & \multicolumn{2}{|l|}{$\mathrm{N}$} & \multicolumn{2}{|l|}{390} & \multicolumn{2}{|l|}{$\mathrm{N}$} & \multicolumn{2}{|l|}{332} \\
\hline & & & \multicolumn{2}{|c|}{$\chi^{2}[\mathrm{df}]$} & \multicolumn{2}{|c|}{3.88 n.s. [2] } & \multicolumn{2}{|c|}{$\chi^{2}[\mathrm{df}]$} & \multicolumn{2}{|c|}{2.09 n.s. [2] } & $\chi^{2}[\mathrm{df}]$ & & 3.30 & \\
\hline & & & CFI & & 1.00 & & CFI & & 1.00 & & CFI & & 1.00 & \\
\hline Messmo & & & TLI & & .99 & & TLI & & 1.00 & & TLI & & 1.00 & \\
\hline & & & RMS & & .05 n.s. & & RMS & & $.01 \mathrm{n}$ & & RMSI & & $.04 \mathrm{n}$ & \\
\hline & & & SRM & & .02 & & SRMI & & .01 & & SRMI & & .02 & \\
\hline
\end{tabular}

** $\mathrm{p} \leq .01 \quad * \mathrm{p} \leq .05 \quad$ n.s. $=$ nicht signifikant 


\subsubsection{Vorteile von Inklusion für Mitschüler*innen}

Kurzbezeichnung Variablenname

Instruktion

Quelle

Weiterführende Literatur

Skalierung (Antwortformat)

Inverse Items (-)

Anzahl Items

Skalenbildung

Instrument xa_gbems/xb_gbems/xc_gbems

(xa_igb_EffD/xb_igb_EffD/xc_igb_EffD)

Wie sehr stimmst du folgenden Aussagen zu?

Bosse \& Spörer, 2014; Gebhardt et al., 2011; Kunz et al., 2010; Paulus, 2013

Werning, 2014; Sturm \& Wagner-Willi, 2018

Endpolbeschriftung; 1 = stimme überhaupt nicht zu, (...), 6 = stimme voll $\mathrm{zu}$

a_igb11, b_igb11, c_igb11

4

per Mittelwert

Hauptfragebogen Messzeitpunkt 1, 2 und 3

\begin{tabular}{|c|c|c|c|}
\hline \multicolumn{4}{|c|}{ Itembezeichnung } \\
\hline MZP1 & MZP2 & MZP3 & Itemwortlaut \\
\hline a_igb8 & b_igb8 & c_igb8 & $\begin{array}{l}\text { Durch Kinder mit geistiger Behinderung lernen die anderen Kinder ver- } \\
\text { stärkt soziale Fähigkeiten (z. B. eina(-) inverse Itemsnder helfen). }\end{array}$ \\
\hline a_igb11 & b_igb11 & c_igb11 & $\begin{array}{l}\text { Die anderen Kinder haben einen Nachteil, weil Kinder mit geistiger Behin- } \\
\text { derung alle Aufmerksamkeit der Lehrkräfte brauchen. (-) }\end{array}$ \\
\hline a_igb13 & b_igb13 & c_igb13 & $\begin{array}{l}\text { Die Anwesenheit von Kindern mit geistiger Behinderung fördert bei den } \\
\text { anderen Kindern die Akzeptanz von Unterschiedlichkeit. }\end{array}$ \\
\hline a_igb14 & b_igb14 & c_igb14 & $\begin{array}{l}\text { Wenn Kinder mit geistiger Behinderung in der Klasse sind, hat das auch für } \\
\text { die anderen Kinder Vorteile, trotz möglicher Schwierigkeiten. }\end{array}$ \\
\hline
\end{tabular}

(-) inverse Items 


\begin{tabular}{|c|c|c|c|c|c|c|c|c|c|c|c|c|c|c|}
\hline \multirow{2}{*}{\multicolumn{3}{|c|}{ Itembezeichnung }} & \multicolumn{12}{|c|}{ Itemkennwerte } \\
\hline & & & \multicolumn{4}{|c|}{ MZP1 } & \multicolumn{4}{|c|}{ MZP2 } & \multicolumn{4}{|c|}{ MZP3 } \\
\hline MZP1 & MZP2 & MZP3 & MW & SD & $\mathrm{r}_{\mathrm{it}}$ & $\lambda_{\mathrm{ij}}$ & MW & $\mathrm{SD}$ & $\mathrm{r}_{\mathrm{it}}$ & $\lambda_{\mathrm{ij}}$ & MW & $\mathrm{SD}$ & $\mathrm{r}_{\mathrm{it}}$ & $\lambda_{\mathrm{ij}}$ \\
\hline a_igb8 & b_igb8 & c_igb8 & 5.03 & 1.18 & .60 & $.76^{* *}$ & 4.95 & 1.21 & .56 & $.77^{* *}$ & 4.93 & 1.15 & .51 & $.73^{* *}$ \\
\hline ra_igb11 & rb_igb11 & rc_igb11 & 3.26 & 1.44 & .30 & $.31^{* *}$ & 3.48 & 1.39 & .16 & $.12^{*}$ & 3.39 & 1.45 & .20 & $.20^{* *}$ \\
\hline a_igb13 & b_igb13 & c_igb13 & 4.90 & 1.21 & .59 & $.81^{* *}$ & 4.91 & 1.23 & .63 & $.91 * *$ & 4.87 & 1.17 & .60 & $.82^{* *}$ \\
\hline a_igb14 & b_igb14 & c_igb14 & 4.31 & 1.31 & .57 & $.68^{* *}$ & 4.40 & 1.27 & .63 & $.68^{* *}$ & 4.36 & 1.29 & .64 & $.71^{* *}$ \\
\hline \multirow{4}{*}{\multicolumn{3}{|c|}{ Skalenkennwerte }} & \multicolumn{2}{|c|}{ Cronbachs $\alpha$} & \multicolumn{2}{|l|}{.74} & \multicolumn{2}{|c|}{ Cronbachs $\alpha$} & \multicolumn{2}{|l|}{.73} & \multicolumn{2}{|c|}{ Cronbachs $\alpha$} & \multicolumn{2}{|l|}{.72} \\
\hline & & & \multicolumn{2}{|l|}{ MW } & \multicolumn{2}{|c|}{4.38} & \multicolumn{2}{|l|}{ MW } & \multicolumn{2}{|c|}{4.43} & \multicolumn{2}{|l|}{ MW } & \multicolumn{2}{|l|}{4.38} \\
\hline & & & \multicolumn{2}{|l|}{$\mathrm{SD}$} & \multicolumn{2}{|l|}{.96} & \multicolumn{2}{|l|}{$\mathrm{SD}$} & \multicolumn{2}{|l|}{.93} & \multicolumn{2}{|l|}{ SD } & \multicolumn{2}{|l|}{.92} \\
\hline & & & \multicolumn{2}{|l|}{$\mathrm{N}$} & \multicolumn{2}{|c|}{431} & \multicolumn{2}{|l|}{$\mathrm{N}$} & \multicolumn{2}{|l|}{391} & \multicolumn{2}{|l|}{$\mathrm{N}$} & \multicolumn{2}{|l|}{332} \\
\hline & & & \multicolumn{2}{|c|}{$\chi^{2}[\mathrm{df}]$} & \multicolumn{2}{|c|}{$12.06^{* *}[2]$} & \multicolumn{2}{|c|}{$\chi^{2}[\mathrm{df}]$} & \multicolumn{2}{|c|}{$23.40 * *[2]$} & $\chi^{2}[\mathrm{~d}$ & & 17.55 & \\
\hline & & & CFI & & .98 & & CFI & & .96 & & CFI & & .95 & \\
\hline Messmoc & & & TLI & & .93 & & TLI & & .87 & & TLI & & .86 & \\
\hline & & & RMSF & & $.11 *$ & & RMS & & $.17^{* *}$ & & RMS & & $.15^{* *}$ & \\
\hline & & & SRMF & & .03 & & SRM & & .05 & & SRM & & .04 & \\
\hline
\end{tabular}

** $\mathrm{p} \leq .01 \quad * \mathrm{p} \leq .05 \quad$ n.s. $=$ nicht signifikant 


\subsubsection{Strukturmodell Einstellungen zu schulischer Inklusion bei geistiger Behinderung}

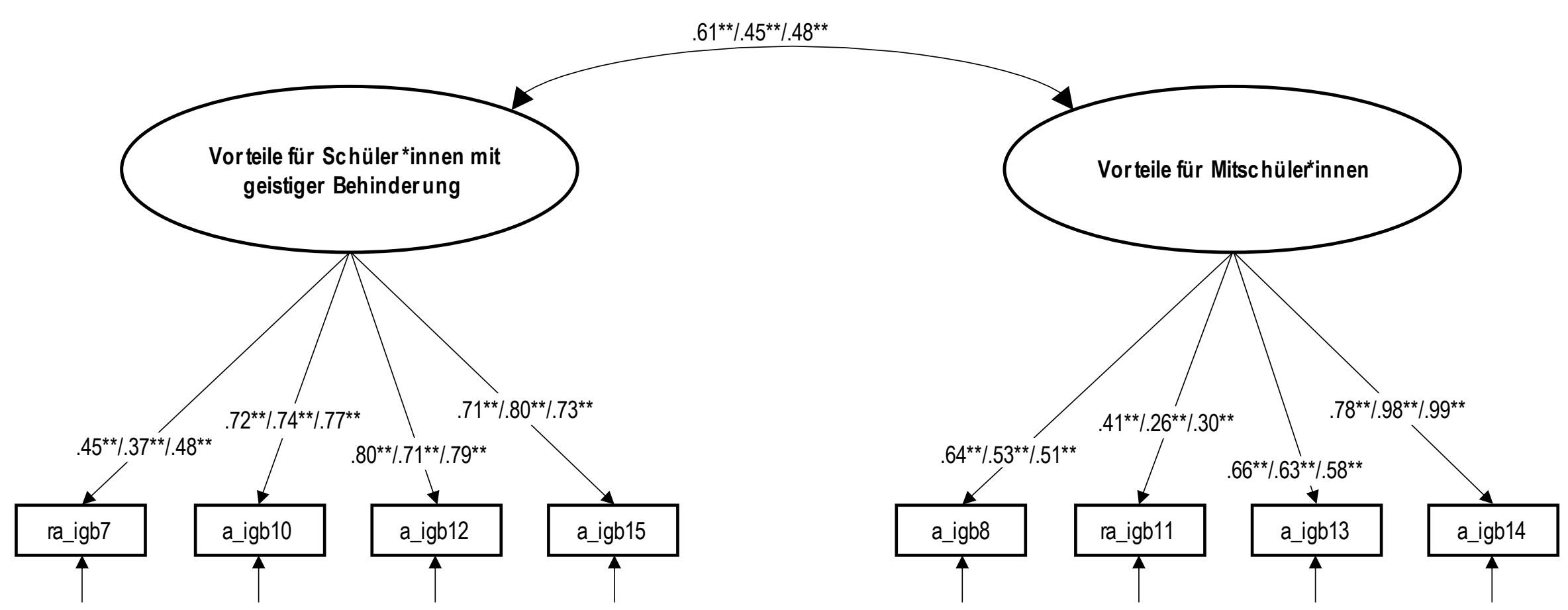

$* * \mathrm{p} \leq .01 \quad * \mathrm{p} \leq .05 \quad$ n.s. $=$ nicht signifikant

Modellfitwerte (MZP1/MZP2/MZP3):

$\chi^{2}[\mathrm{df}]=54.21^{*}[17] / 106.03 * *[17] / 57.63 *[17] ; \mathrm{CFI}=.97 / .91 / .95 ; \mathrm{TLI}=.94 / .86 / .92 ; \mathrm{TLI}=.94 / .86 / .92 ; \mathrm{RMSEA}=.07 * / .11 * * / .09 * * ; \mathrm{SRMR}=.05 / .08 / .06$ 



\subsubsection{Schulische Inklusion von Schüler*innen mit psychischer Erkrankung}

\subsubsection{Vorteile von Inklusion für Scbüler*innen mit psychischer Erkerankung}

Kurzbezeichnung Variablenname

Instruktion

Quelle

Weiterführende Literatur

Skalierung (Antwortformat)

Inverse Items (-)

Anzahl Items

Skalenbildung

Instrument xa_pbesb/xb_pbesb/xc_pbesb
(xa_ipb_EffB/xb_ipb_EffB/xc_ipb_EffB)

Zuletzt geht es um Einschätzungen dazu, wenn Kinder psychischer Erkrankung auf Regelschulen gehen. Wie sehr stimmst du folgenden Aussagen zu?

Gebhardt et al., 2011; Kunz et al., 2010; Paulus, 2013

Werning, 2014; Sturm \& Wagner-Willi, 2018

Endpolbeschriftung; 1 = stimme überhaupt nicht zu, (...), 6 = stimme voll $\mathrm{zu}$

a_ipb7, b_ipb7, c_ipb7

4

per Mittelwert

Hauptfragebogen Messzeitpunkt 1, 2 und 3

\begin{tabular}{llll}
\hline $\begin{array}{l}\text { Itembezeichnung } \\
\text { MZP1 }\end{array}$ & MZP2 & MZP3 & Itemwortlaut \\
a_ipb7 & b_ipb7 & c_ipb7 & $\begin{array}{l}\text { Kinder mit psychischer Erkrankung würden in Förderschulen mehr lernen. } \\
(-)\end{array}$ \\
a_ipb10 & b_ipb10 & c_ipb10 & $\begin{array}{l}\text { Kinder mit psychischer Erkrankung können in Regelschulen besser zu } \\
\text { selbstständigem Lernen angeregt werden als in Förderschulen. }\end{array}$ \\
a_ipb12 & b_ipb12 & c_ipb12 & $\begin{array}{l}\text { Kinder mit psychischer Erkrankung entwickeln in Regelschulen ein größe- } \\
\text { res Selbstbewusstsein als in Förderschulen. }\end{array}$ \\
a_ipb15 & b_ipb15 & c_ipb15 & $\begin{array}{l}\text { Kinder mit psychischer Erkrankung lernen in Regelschulen wichtige Dinge, } \\
\text { die sie in Förderschulen nicht lernen würden. }\end{array}$ \\
\hline$(-)$ invers
\end{tabular}

(-) inverse Items 


\begin{tabular}{|c|c|c|c|c|c|c|c|c|c|c|c|c|c|c|}
\hline \multirow{2}{*}{\multicolumn{3}{|c|}{ Itembezeichnung }} & \multicolumn{12}{|c|}{ Itemkennwerte } \\
\hline & & & \multicolumn{4}{|c|}{ MZP1 } & \multicolumn{4}{|c|}{ MZP2 } & \multicolumn{4}{|c|}{ MZP3 } \\
\hline MZP1 & MZP2 & MZP3 & MW & $\mathrm{SD}$ & $\mathrm{r}_{\mathrm{it}}$ & $\lambda_{\mathrm{ij}}$ & MW & SD & $\mathrm{r}_{\mathrm{it}}$ & $\lambda_{\mathrm{ij}}$ & MW & $\mathrm{SD}$ & $\mathrm{r}_{\mathrm{it}}$ & $\lambda_{\mathrm{ij}}$ \\
\hline ra_ipb7 & rb_ipb7 & rc_ipb7 & 3.87 & 1.47 & .48 & $.54 * *$ & 3.87 & 1.39 & .45 & $.53^{* *}$ & 3.82 & 1.47 & .40 & $.43^{* *}$ \\
\hline a_ipb10 & b_ipb10 & c_ipb10 & 3.68 & 1.33 & .67 & $.80 * *$ & 3.69 & 1.30 & .66 & $.78^{* *}$ & 3.89 & 1.32 & .73 & $.95^{* *}$ \\
\hline a_ipb12 & b_ipb12 & c_ipb12 & 3.53 & 1.30 & .57 & $.68^{* *}$ & 3.69 & 1.29 & .53 & $.65^{* *}$ & 3.63 & 1.26 & .52 & $.64^{* *}$ \\
\hline a_ipb15 & b_ipb15 & c_ipb15 & 3.97 & 1.45 & .64 & $.75^{* *}$ & 3.98 & 1.40 & .67 & $.78^{* *}$ & 3.97 & 1.41 & .56 & $.64^{* *}$ \\
\hline \multirow{4}{*}{\multicolumn{3}{|c|}{ Skalenkennwerte }} & \multicolumn{2}{|c|}{ Cronbachs $\alpha$} & \multicolumn{2}{|l|}{.78} & \multicolumn{2}{|c|}{ Cronbachs $\alpha$} & \multicolumn{2}{|l|}{.78} & \multicolumn{2}{|c|}{ Cronbachs $\alpha$} & \multicolumn{2}{|l|}{.76} \\
\hline & & & \multicolumn{2}{|l|}{ MW } & \multicolumn{2}{|l|}{3.77} & \multicolumn{2}{|l|}{ MW } & \multicolumn{2}{|l|}{3.81} & \multicolumn{2}{|l|}{ MW } & \multicolumn{2}{|l|}{3.83} \\
\hline & & & \multicolumn{2}{|l|}{$\mathrm{SD}$} & \multicolumn{2}{|c|}{1.08} & \multicolumn{2}{|l|}{$\mathrm{SD}$} & \multicolumn{2}{|l|}{1.05} & \multicolumn{2}{|l|}{$\mathrm{SD}$} & \multicolumn{2}{|l|}{1.05} \\
\hline & & & \multicolumn{2}{|l|}{$\mathrm{N}$} & \multicolumn{2}{|l|}{422} & \multicolumn{2}{|l|}{$\mathrm{N}$} & \multicolumn{2}{|l|}{387} & \multicolumn{2}{|l|}{$\mathrm{N}$} & \multicolumn{2}{|l|}{328} \\
\hline & & & \multicolumn{2}{|c|}{$\chi^{2}[\mathrm{df}]$} & \multicolumn{2}{|c|}{5.06 n.s. [2] } & \multicolumn{2}{|c|}{$\chi^{2}[\mathrm{df}]$} & \multicolumn{2}{|c|}{$10.55^{* *}[2]$} & $\chi^{2}[\mathrm{~d} f$ & & 2.42 & \\
\hline & & & CFI & & .99 & & CFI & & .98 & & CFI & & 1.00 & \\
\hline Messmoc & & & TLI & & .98 & & TLI & & .94 & & TLI & & 1.00 & \\
\hline & & & RMSF & & $.06 \mathrm{n}$ & & RMS & & .11 n.s. & & RMS & & $.03 \mathrm{n}$ & \\
\hline & & & SRMF & & .02 & & SRM & & .03 & & SRM & & .02 & \\
\hline
\end{tabular}

$* * \mathrm{p} \leq .01 \quad * \mathrm{p} \leq .05 \quad$ n.s. $=$ nicht signifikant 


\subsubsection{Vorteile von Inklusion für Mitschüler*innen}

Kurzbezeichnung Variablenname

Instruktion

Quelle

Weiterführende Literatur

Skalierung (Antwortformat)

Inverse Items (-)

Anzahl Items

Skalenbildung

Instrument xa_pbems/xb_pbems/xc_pbems

(xa_ipb_EffD/xb_ipb_EffD/xc_ipb_EffD)

Wie sehr stimmst du folgenden Aussagen zu?

Bosse \& Spörer, 2014; Gebhardt et al., 2011; Kunz et al., 2010; Paulus, 2013

Werning, 2014; Sturm \& Wagner-Willi, 2018

Endpolbeschriftung; 1 = stimme überhaupt nicht zu, (...), $6=$ stimme voll $\mathrm{zu}$

a_ipb11, b_ipb11, c_ipb11

per Mittelwert

Hauptfragebogen Messzeitpunkt 1, 2 und 3

\begin{tabular}{|c|c|c|c|}
\hline \multicolumn{4}{|c|}{ Itembezeichnung } \\
\hline MZP1 & MZP2 & MZP3 & Itemwortlaut \\
\hline a_ipb8 & b_ipb8 & c_ipb8 & $\begin{array}{l}\text { Durch Kinder mit psychischer Erkrankung lernen die anderen Kinder } \\
\text { verstärkt soziale Fähigkeiten (z. B. einander helfen). }\end{array}$ \\
\hline a_ipb11 & b_ipb11 & c_ipb11 & $\begin{array}{l}\text { Die anderen Kinder haben einen Nachteil, weil Kinder mit psychischer } \\
\text { Erkrankung alle Aufmerksamkeit der Lehrkräfte brauchen. (-) }\end{array}$ \\
\hline a_ipb13 & b_ipb13 & c_ipb13 & $\begin{array}{l}\text { Die Anwesenheit von Kindern mit psychischer Erkrankung fördert bei den } \\
\text { anderen Kindern die Akzeptanz von Unterschiedlichkeit. }\end{array}$ \\
\hline a_ipb14 & b_ipb14 & c_ipb14 & $\begin{array}{l}\text { Wenn Kinder mit psychischer Erkrankung in der Klasse sind, hat das auch } \\
\text { für die anderen Kinder Vorteile, trotz möglicher Schwierigkeiten. }\end{array}$ \\
\hline
\end{tabular}

(-) inverse Items 


\begin{tabular}{|c|c|c|c|c|c|c|c|c|c|c|c|c|c|c|}
\hline \multirow{2}{*}{\multicolumn{3}{|c|}{ Itembezeichnung }} & \multicolumn{12}{|c|}{ Itemkennwerte } \\
\hline & & & \multicolumn{4}{|c|}{ MZP1 } & \multicolumn{4}{|c|}{ MZP2 } & \multicolumn{4}{|c|}{ MZP3 } \\
\hline MZP1 & MZP2 & MZP3 & MW & $\mathrm{SD}$ & $\mathrm{r}_{\mathrm{it}}$ & $\lambda_{\mathrm{ij}}$ & MW & SD & $\mathrm{r}_{\mathrm{it}}$ & $\lambda_{\mathrm{ij}}$ & MW & $\mathrm{SD}$ & $\mathrm{r}_{\mathrm{it}}$ & $\lambda_{\mathrm{ij}}$ \\
\hline a_ipb8 & b_ipb8 & c_ipb8 & 4.72 & 1.27 & .61 & $.81 * *$ & 4.62 & 1.27 & .56 & $.74^{* *}$ & 4.67 & 1.21 & .56 & $.77 * *$ \\
\hline ra_ipb11 & rb_ipb11 & rc_ipb11 & 4.00 & 1.43 & .20 & $.18^{* *}$ & 4.03 & 1.33 & .18 & $.20^{* *}$ & 3.92 & 1.34 & .19 & $.19 * *$ \\
\hline a_ipb13 & b_ipb13 & c_ipb13 & 4.72 & 1.29 & .61 & $.82^{* *}$ & 4.57 & 1.30 & .63 & $.82^{* *}$ & 4.49 & 1.29 & .60 & $.81 * *$ \\
\hline a_ipb14 & b_ipb14 & c_ipb14 & 4.21 & 1.36 & .64 & $.72 * *$ & 4.20 & 1.36 & .63 & $.75^{* *}$ & 4.18 & 1.26 & .57 & $.66^{* *}$ \\
\hline \multirow{4}{*}{\multicolumn{3}{|c|}{ Skalenkennwerte }} & \multicolumn{2}{|c|}{ Cronbachs $\alpha$} & \multicolumn{2}{|l|}{.73} & \multicolumn{2}{|c|}{ Cronbachs $\alpha$} & \multicolumn{2}{|l|}{.72} & \multicolumn{2}{|c|}{ Cronbachs $\alpha$} & .71 & \\
\hline & & & \multicolumn{2}{|l|}{ MW } & \multicolumn{2}{|c|}{4.40} & \multicolumn{2}{|c|}{ MW } & \multicolumn{2}{|l|}{4.37} & \multicolumn{2}{|l|}{ MW } & \multicolumn{2}{|l|}{4.32} \\
\hline & & & \multicolumn{2}{|l|}{$\mathrm{SD}$} & \multicolumn{2}{|l|}{.98} & \multicolumn{2}{|l|}{$\mathrm{SD}$} & \multicolumn{2}{|l|}{.96} & \multicolumn{2}{|l|}{$\mathrm{SD}$} & \multicolumn{2}{|l|}{.91} \\
\hline & & & \multicolumn{2}{|l|}{$\mathrm{N}$} & \multicolumn{2}{|l|}{421} & \multicolumn{2}{|l|}{$\mathrm{N}$} & \multicolumn{2}{|l|}{383} & \multicolumn{2}{|l|}{$\mathrm{N}$} & \multicolumn{2}{|l|}{328} \\
\hline & & & \multicolumn{2}{|c|}{$\chi^{2}[\mathrm{df}]$} & \multicolumn{2}{|c|}{$15.37^{* *}[2]$} & \multicolumn{2}{|c|}{$\chi^{2}[\mathrm{df}]$} & \multicolumn{2}{|c|}{$7.86^{*}[2]$} & \multicolumn{2}{|c|}{$\chi^{2}[\mathrm{df}]$} & $6.87 *[2]$ & \\
\hline & & & CFI & & .97 & & CFI & & .99 & & CFI & & .98 & \\
\hline Messmod & & & TLI & & .91 & & TLI & & .96 & & TLI & & .95 & \\
\hline & & & RMSF & & $.13^{*}$ & & RMS & & .09 n.s. & & RMS & & .09 n.s. & \\
\hline & & & SRMF & & .03 & & SRM & & .03 & & SRMI & & .03 & \\
\hline
\end{tabular}

** $\mathrm{p} \leq .01 \quad * \mathrm{p} \leq .05 \quad$ n.s. $=$ nicht signifikant 
2.2.3.3 Strukturmodell Einstellungen zu schulischer Inklusion bei psychischer Erkerankung

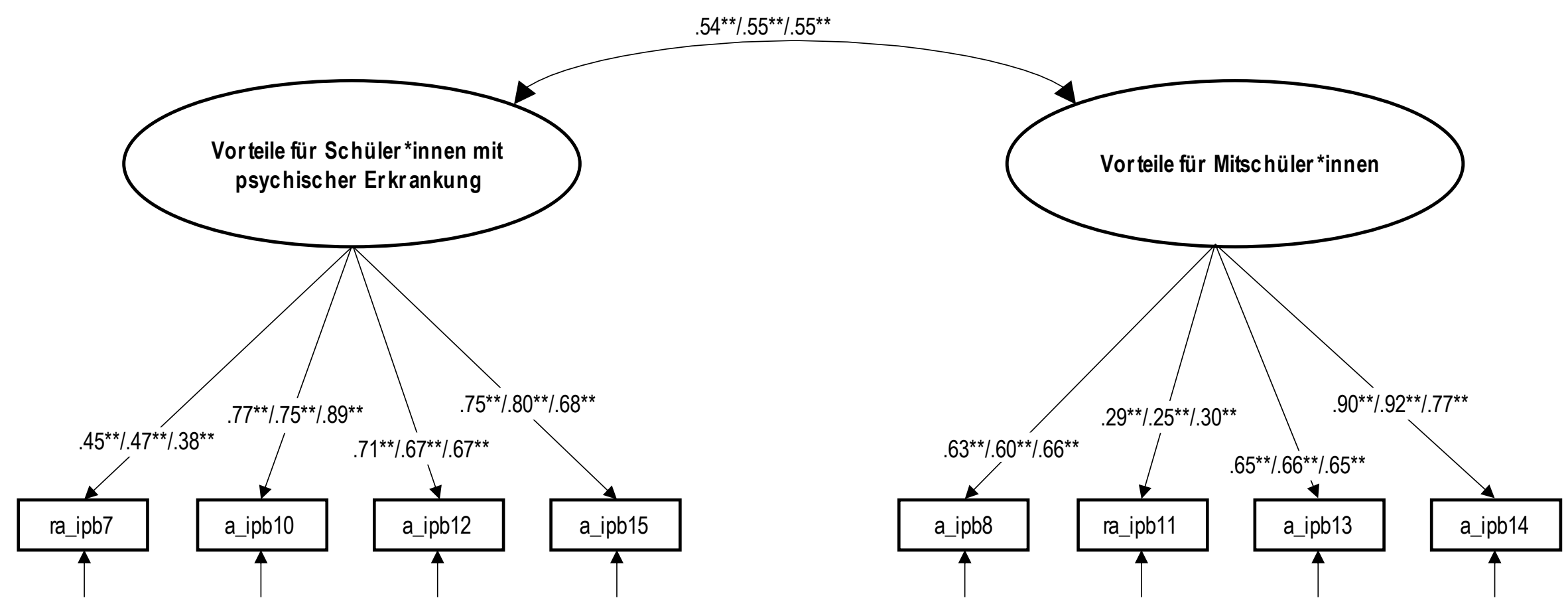

$* * \mathrm{p} \leq .01 \quad * \mathrm{p} \leq .05 \quad$ n.s. $=$ nicht signifikant

Modellfitwerte (MZP1/MZP2/MZP3):

$\chi^{2}[\mathrm{df}]=59.09^{* *}[17] / 61.94^{* *}[17] / 69.45^{* *}[17] ; \mathrm{CFI}=.96 / .96 / .94 ; \mathrm{TLI}=.94 / .93 / .90 ; \mathrm{TLI}=.94 / .93 / .90 ; \mathrm{RMSEA}=.08^{*} / .08^{* *} / .10^{* *} ; \mathrm{SRMR}=.06 / .06 / .06$ 


\subsection{Befürwortung von Inklusion bei spezifischer Behinderung}

Kurzbezeichnung Variablenname

Instruktion

Quelle

Skalierung (Antwortformat)

Instrument a_rfs2/b_rfs2/c_rfs2

LVorherige offene Frage: Nun möchten wir dich bitten, auf der Linie eine Behinderungsart oder psychische Krankheit einzutragen.

Du machst dein FSJ/BFD im Bereich Menschen mit Behinderung/psychischer Erkrankung? Dann trage eine Behinderungsart oder psychische Krankheit ein, die du von der Einsatzstelle kennst (wenn du noch nicht gearbeitet hast, setze eine ein, von der du denkst, dass du sie in deiner Einsatzstelle kennenlernen wirst).

Du machst dein FSJ/BFD in einem anderen Bereich? Dann setze einfach eine Behinderungsart oder psychische Krankheit ein, die du (gut) kennst.]

Sollte ein Kind, das diese Behinderung/Krankheit hat, deiner Meinung nach eine Regelschule oder eine Förderschule besuchen?

Eigenentwicklung

Endpolbeschriftung; 1 = Auf jeden Fall eine Regelschule, (...), 5 = Auf jeden Fall eine Förderschule

Hauptfragebogen Messzeitpunkt 1, 2 und 3

\begin{tabular}{|c|c|c|c|c|c|c|c|c|c|c|c|c|c|c|c|}
\hline \multirow[t]{3}{*}{ Itembezeichnung } & \multirow{2}{*}{\multicolumn{2}{|c|}{$\begin{array}{l}\text { Ausprägung } \\
1\end{array}$}} & \multirow{2}{*}{\multicolumn{2}{|c|}{$\begin{array}{l}\text { Ausprägung } \\
2\end{array}$}} & \multirow{2}{*}{\multicolumn{2}{|c|}{$\begin{array}{l}\text { Ausprägung } \\
3\end{array}$}} & \multirow{2}{*}{\multicolumn{2}{|c|}{$\begin{array}{l}\text { Ausprägung } \\
4\end{array}$}} & \multirow{2}{*}{\multicolumn{2}{|c|}{$\begin{array}{l}\text { Ausprägung } \\
5\end{array}$}} & \multirow{2}{*}{\multicolumn{2}{|c|}{ Fehlende Werte }} & \multirow{2}{*}{\multicolumn{3}{|c|}{ Gesamt }} \\
\hline & & & & & & & & & & & & & & & \\
\hline & $\mathrm{N}$ & $\%$ & $\mathrm{~N}$ & $\%$ & $\mathrm{~N}$ & $\%$ & $\mathrm{~N}$ & $\%$ & $\mathrm{~N}$ & $\%$ & $\mathrm{~N}$ & $\%$ & $\mathrm{~N}$ & MW & $\mathrm{SD}$ \\
\hline a_rfs2 & 56 & 12.58 & 83 & 18.65 & 120 & 26.97 & 100 & 22.47 & 64 & 14.38 & 22 & 4.94 & 445 & 3.08 & 1.25 \\
\hline b_rfs2 & 78 & 18.84 & 66 & 15.94 & 114 & 27.54 & 73 & 17.63 & 60 & 14.49 & 23 & 5.56 & 414 & 2.93 & 1.33 \\
\hline c_rfs2 & 53 & 15.41 & 67 & 19.48 & 96 & 27.91 & 70 & 20.35 & 38 & 11.05 & 20 & 5.81 & 344 & 2.92 & 1.24 \\
\hline
\end{tabular}




\section{Arbeitsbezogene Selbstwirksamkeitserwartung}

Kurzbezeichnung Variablenname

Instruktion

Quelle

Weiterführende Literatur

Skalierung (Antwortformat)

Inverse Items (-)

Anzahl Items

Skalenbildung

Instrument xa_swe/xb_swe/xc_swe

Wie sehr stimmst du folgender Aussage zu?

Schwarzer, 1999

Jerusalem \& Hopf, 2002; Schmitz \& Schwarzer, 1999

Endpolbeschriftung; 1 = stimme überhaupt nicht zu, $(\ldots), 6=$ stimme voll $\mathrm{zu}$

a_swe7, b_swe7, c_swe7

10

per Mittelwert

Hauptfragebogen Messzeitpunkt 1, 2 und 3

\begin{tabular}{|c|c|c|c|}
\hline \multicolumn{4}{|c|}{ Itembezeichnung } \\
\hline MZP1 & MZP2 & MZP3 & Itemwortlaut \\
\hline a_swe1 & b_swe1 & c_swe1 & $\begin{array}{l}\text { Ich weiß, dass ich es schaffe, selbst Menschen mit den schwersten Behinde- } \\
\text { rungen etwas zu vermitteln. }\end{array}$ \\
\hline a_swe2 & b_swe2 & c_swe2 & $\begin{array}{l}\text { Ich weiß, dass ich zu Menschen mit Behinderung guten Kontakt aufbauen } \\
\text { kann, selbst in schwierigen Situationen. }\end{array}$ \\
\hline a_swe3 & b_swe3 & c_swe3 & $\begin{array}{l}\text { Ich bin mir sicher, dass ich auch mit Menschen mit sehr starker Behinde- } \\
\text { rung in guten Kontakt kommen kann, wenn ich mich darum bemühe. }\end{array}$ \\
\hline a_swe4 & b_swe4 & c_swe4 & $\begin{array}{l}\text { Ich bin mir sicher, dass ich mich in Zukunft auf individuelle Probleme von } \\
\text { Menschen mit Behinderung noch besser einstellen kann. }\end{array}$ \\
\hline a_swe5 & b_swe5 & c_swe 5 & $\begin{array}{l}\text { Selbst wenn bei meiner Arbeit mit Menschen mit Behinderung etwas nicht } \\
\text { so läuft wie geplant, bin ich mir sicher, die notwendige Gelassenheit bewah- } \\
\text { ren zu können. }\end{array}$ \\
\hline a_swe6 & b_swe6 & c_swe6 & $\begin{array}{l}\text { Selbst wenn es mir mal nicht so gut geht, kann ich doch bei der Arbeit } \\
\text { immer noch gut auf die Menschen mit Behinderung eingehen. }\end{array}$ \\
\hline a_swe7 & b_swe7 & c_swe7 & $\begin{array}{l}\text { Auch wenn ich mich noch so sehr für die Entwicklung der Menschen mit } \\
\text { Behinderung engagiere, weiß ich, dass ich nicht viel ausrichten kann. (-) }\end{array}$ \\
\hline a_swe8 & b_swe8 & c_swe8 & $\begin{array}{l}\text { Ich bin mir sicher, dass ich kreative Ideen entwickeln kann, mit denen ich } \\
\text { ungünstige Strukturen bei der Arbeit mit Menschen mit Behinderung ver- } \\
\text { ändern kann. }\end{array}$ \\
\hline
\end{tabular}




\begin{tabular}{llll}
\multicolumn{1}{l}{$\begin{array}{l}\text { Itembezeichnung } \\
\text { MZP1 }\end{array}$} & MZP2 & MZP3 & Itemwortlaut \\
\hline a_swe9 & b_swe9 & c_swe9 & $\begin{array}{l}\text { Ich traue mir zu, Menschen mit Behinderung für neue Projekte bei der } \\
\text { Arbeit zu begeistern. }\end{array}$ \\
a_swe10 & b_swe10 & c_swe10 & $\begin{array}{l}\text { Ich kann Veränderungen im Rahmen meiner Arbeit mit Menschen mit } \\
\text { Behinderung auch gegenüber skeptischen Kollegen durchsetzen. }\end{array}$ \\
\hline
\end{tabular}

(-) inverse Items 


\begin{tabular}{|c|c|c|c|c|c|c|c|c|c|c|c|c|c|c|}
\hline \multirow{2}{*}{\multicolumn{3}{|c|}{ Itembezeichnung }} & \multicolumn{12}{|c|}{ Itemkennwerte } \\
\hline & & & \multicolumn{4}{|c|}{ MZP1 } & \multicolumn{4}{|c|}{ MZP2 } & \multicolumn{4}{|c|}{ MZP3 } \\
\hline MZP1 & MZP2 & MZP3 & MW & SD & $\mathrm{r}_{\mathrm{it}}$ & $\lambda_{\mathrm{ij}}$ & MW & SD & $\mathrm{r}_{\mathrm{it}}$ & $\lambda_{\mathrm{ij}}$ & MW & SD & $r_{i t}$ & $\lambda_{\mathrm{ij}}$ \\
\hline a_swe1 & b_swe1 & c_swe1 & 4.14 & 1.36 & .55 & $.63^{* *}$ & 4.42 & 1.27 & .67 & $.72 * *$ & 4.56 & 1.24 & .64 & $.66^{* *}$ \\
\hline a_swe2 & b_swe2 & c_swe2 & 4.47 & 1.14 & .72 & $.76^{* *}$ & 4.58 & 1.22 & .72 & $.78^{* *}$ & 4.72 & 1.19 & .73 & $.76^{* *}$ \\
\hline a_swe3 & b_swe3 & c_swe3 & 4.75 & 1.19 & .72 & $.78^{* *}$ & 4.89 & 1.08 & .72 & $.78^{* *}$ & 4.86 & 1.16 & .68 & $.74 * *$ \\
\hline a_swe4 & b_swe4 & c_swe4 & 4.88 & 1.06 & .63 & $.66^{* *}$ & 4.89 & 1.05 & .73 & $.78^{* *}$ & 4.89 & 1.11 & .77 & $.84 * *$ \\
\hline a_swe5 & b_swe 5 & c_swe5 & 4.61 & 1.11 & .61 & $.65^{* *}$ & 4.61 & 1.15 & .63 & $.69 * *$ & 4.72 & 1.11 & .69 & $.75^{* *}$ \\
\hline a_swe6 & b_swe6 & c_swe6 & 4.52 & 1.19 & .56 & $.61 * *$ & 4.60 & 1.20 & .73 & $.77 * *$ & 4.74 & 1.16 & .71 & $.78^{* *}$ \\
\hline ra_swe7 & rb_swe7 & rc_swe7 & 4.07 & 1.27 & .17 & $.21 * *$ & 4.01 & 1.34 & .23 & $.24 * *$ & 3.93 & 1.34 & .20 & $.13^{*}$ \\
\hline a_swe8 & b_swe8 & c_swe8 & 3.99 & 1.23 & .54 & $.56^{* *}$ & 4.14 & 1.17 & .59 & $.62 * *$ & 4.13 & 1.29 & .56 & $.58^{* *}$ \\
\hline a_swe9 & b_swe9 & c_swe9 & 4.51 & 1.18 & .65 & $.69 * *$ & 4.49 & 1.16 & .68 & $.71 * *$ & 4.54 & 1.25 & .75 & $.78^{* *}$ \\
\hline a_swe10 & b_swe10 & c_swe10 & 3.89 & 1.22 & .57 & $.61 * *$ & 4.02 & 1.14 & .64 & $.67 * *$ & 4.25 & 1.15 & .63 & $.65^{* *}$ \\
\hline \multirow{4}{*}{\multicolumn{3}{|c|}{ Skalenkennwerte }} & \multicolumn{2}{|c|}{ Cronbachs $\alpha$} & \multicolumn{2}{|l|}{.87} & \multicolumn{2}{|c|}{ Cronbachs $\alpha$} & \multicolumn{2}{|l|}{.90} & \multicolumn{2}{|c|}{ Cronbachs $\alpha$} & \multicolumn{2}{|l|}{.90} \\
\hline & & & \multicolumn{2}{|l|}{ MW } & \multicolumn{2}{|l|}{4.40} & \multicolumn{2}{|l|}{ MW } & \multicolumn{2}{|l|}{4.47} & \multicolumn{2}{|l|}{ MW } & \multicolumn{2}{|l|}{4.53} \\
\hline & & & \multicolumn{2}{|l|}{$\mathrm{SD}$} & \multicolumn{2}{|l|}{.79} & \multicolumn{2}{|l|}{$\mathrm{SD}$} & \multicolumn{2}{|l|}{.83} & \multicolumn{2}{|l|}{$\mathrm{SD}$} & \multicolumn{2}{|l|}{.88} \\
\hline & & & \multicolumn{2}{|l|}{$\mathrm{N}$} & \multicolumn{2}{|l|}{314} & \multicolumn{2}{|l|}{$\mathrm{N}$} & \multicolumn{2}{|l|}{307} & \multicolumn{2}{|l|}{$\mathrm{N}$} & \multicolumn{2}{|l|}{279} \\
\hline & & & \multicolumn{2}{|c|}{$\chi^{2}[\mathrm{df}]$} & 143. & [35] & $\chi^{2}[\mathrm{~d}$ & & 135.1 & [35] & $\chi^{2}[\mathrm{df}]$ & & 170.1 & 35] \\
\hline & & & CFI & & .90 & & CFI & & .93 & & CFI & & .90 & \\
\hline Messmod & & & TLI & & .86 & & TLI & & .91 & & TLI & & .87 & \\
\hline & & & RMSI & & $.10^{* *}$ & & RMS & & $.10^{* *}$ & & RMSF & & $.12^{* *}$ & \\
\hline & & & SRMI & & .05 & & SRM & & .04 & & SRMF & & .05 & \\
\hline
\end{tabular}





\section{Wissen zu Inklusion}

\subsection{Inklusive Gesellschaft}

Kurzbezeichnung Variablenname

Instruktion

Quelle

Skalierung (Antwortformat)

Instrument a_w_i/b_w_i/c_w_i

Kannst du dir unter einer inklusiven Gesellschaft etwas vorstellen?

Eigenentwicklung

Dichotom; $0=$ Nein; $1=\mathrm{Ja}$

Hauptfragebogen Messzeitpunkt 1, 2 und 3

\begin{tabular}{lllllllll}
\hline & Itembezeichnung & Ausprägung & \multicolumn{2}{l}{ Ausprägung } & \multicolumn{2}{c}{ Fehlende Werte } & Gesamt \\
& & 0 & & 1 & & & & \\
& & $\mathrm{~N}$ & $\%$ & $\mathrm{~N}$ & $\%$ & $\mathrm{~N}$ & $\%$ & $\mathrm{~N}$ \\
\hline MZP1 & a_w_i & 170 & 38.20 & 194 & 43.60 & 81 & 18.20 & 445 \\
MZP2 & b_w_i & 150 & 36.23 & 229 & 55.31 & 35 & 8.45 & 414 \\
MZP3 & c_w_i & 100 & 29.07 & 208 & 60.47 & 36 & 10.47 & 344 \\
\hline
\end{tabular}




\subsection{UN-Behindertenrechtskonvention}

Kurzbezeichnung Variablenname

Instruktion

Quelle

Skalierung (Antwortformat)

Instrument a_w_brk/b_w_brk/c_w_brk

Kennst du die UN-Behindertenrechtskonvention?

Eigenentwicklung

Dichotom; 0 = Nein; $1=\mathrm{Ja}$

Hauptfragebogen Messzeitpunkt 1, 2 und 3

\begin{tabular}{|c|c|c|c|c|c|c|c|c|}
\hline & \multirow[t]{3}{*}{ Itembezeichnung } & \multirow{2}{*}{\multicolumn{2}{|c|}{$\begin{array}{l}\text { Ausprägung } \\
0\end{array}$}} & \multirow{2}{*}{\multicolumn{2}{|c|}{$\begin{array}{l}\text { Ausprägung } \\
1\end{array}$}} & \multicolumn{2}{|c|}{ Fehlende Werte } & \multirow[t]{2}{*}{ Gesam } \\
\hline & & & & & & & & \\
\hline & & $\mathrm{N}$ & $\%$ & $\mathrm{~N}$ & $\%$ & $\mathrm{~N}$ & $\%$ & $\mathrm{~N}$ \\
\hline MZP1 & a_w_brk & 387 & 86.97 & 36 & 8.09 & 22 & 4.94 & 445 \\
\hline MZP2 & b_w_brk & 331 & 79.95 & 56 & 13.53 & 27 & 6.52 & 414 \\
\hline MZP3 & c_w_brk & 274 & 79.65 & 52 & 15.11 & 18 & 5.23 & 344 \\
\hline
\end{tabular}




\subsection{Exklusion/Separation/Integration/Inklusion}

Kurzbezeichnung Variablenname

Instruktion

Quelle

Antwortformat a_w_gr/b_w_gr/c_w_gr

Die folgenden Abbildungen veranschaulichen verschiedene Möglichkeiten, wie in einer Gesellschaft mit Menschen mit Behinderung umgegangen wird. Versuche bitte, die Abbildungen den Begriffen unten zuzuordnen, indem du die Nummer der Abbildung [siehe Grafik] in das Kästchen neben den Begriff schreibst.

Lorenz, Stubbe, Krieg \& Renftel, 2020

Zuordnungsaufgabe, siehe untenstehende Grafik

Hauptfragebogen Messzeitpunkt 1, 2, und 3

\begin{tabular}{llll}
\hline \multicolumn{2}{l}{ Itembezeichnung } & & \\
MZP1 & MZP2 & MZP3 & Itemwortlaut \\
\hline a_w_gr1 & b_w_gr1 & c_w_gr1 & Exklusion \\
a_w_gr2 & b_w_gr2 & c_w_gr2 & Inklusion \\
a_w_gr3 & b_w_gr3 & c_w_gr3 & Integration \\
a_w_gr4 & b_w_gr4 & c_w_gr4 & Separation \\
\hline
\end{tabular}

1
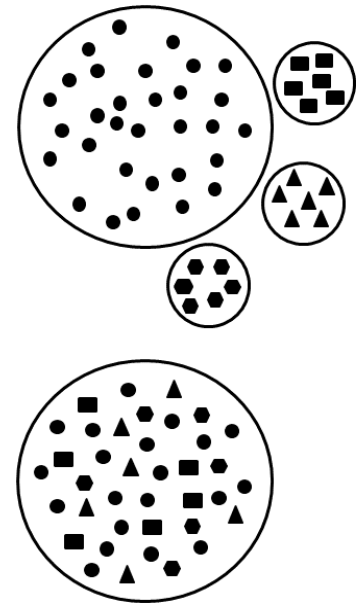

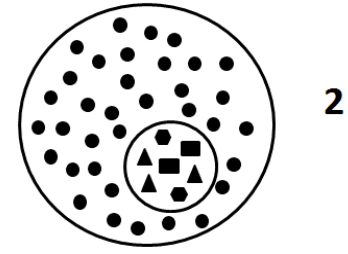

2

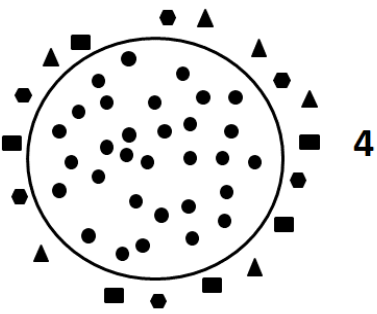




\begin{tabular}{|c|c|c|c|c|c|c|c|c|c|c|c|}
\hline \multirow{3}{*}{$\begin{array}{l}\text { Itembezeichnung } \\
\text { MZP1 }\end{array}$} & \multirow{2}{*}{\multicolumn{2}{|c|}{$\begin{array}{l}\text { Ausprägung } \\
1\end{array}$}} & \multirow{2}{*}{\multicolumn{2}{|c|}{$\begin{array}{l}\text { Ausprägung } \\
2\end{array}$}} & \multirow{2}{*}{\multicolumn{2}{|c|}{$\begin{array}{l}\text { Ausprägung } \\
3\end{array}$}} & \multirow{2}{*}{\multicolumn{2}{|c|}{$\begin{array}{l}\text { Ausprägung } \\
4\end{array}$}} & \multirow{2}{*}{\multicolumn{2}{|c|}{ Fehlende Werte }} & \multirow{3}{*}{$\begin{array}{l}\text { Gesamt } \\
\mathrm{N}\end{array}$} \\
\hline & & & & & & & & & & & \\
\hline & $\mathrm{N}$ & $\%$ & $\mathrm{~N}$ & $\%$ & $\mathrm{~N}$ & $\%$ & $\mathrm{~N}$ & $\%$ & $\mathrm{~N}$ & $\%$ & \\
\hline a_w_gr1 & 64 & 14.38 & 21 & 4.72 & 5 & 1.12 & 329 & 73.93 & 26 & 5.84 & 445 \\
\hline a_w_gr2 & 9 & 2.02 & 206 & 46.29 & 180 & 40.45 & 24 & 5.39 & 26 & 5.84 & 445 \\
\hline a_w_gr3 & 5 & 1.12 & 170 & 38.20 & 234 & 52.58 & 9 & 2.02 & 27 & 6.07 & 445 \\
\hline a_w_gr4 & 341 & 76.63 & 18 & 4.04 & 2 & .45 & 57 & 12.81 & 27 & 6.07 & 445 \\
\hline
\end{tabular}

\begin{tabular}{|c|c|c|c|c|c|c|c|c|c|c|c|}
\hline \multirow{3}{*}{$\begin{array}{l}\text { Itembezeichnung } \\
\text { MZP2 }\end{array}$} & \multirow{2}{*}{\multicolumn{2}{|c|}{$\begin{array}{l}\text { Ausprägung } \\
1\end{array}$}} & \multirow{2}{*}{\multicolumn{2}{|c|}{$\begin{array}{l}\text { Ausprägung } \\
2\end{array}$}} & \multirow{2}{*}{\multicolumn{2}{|c|}{$\begin{array}{l}\text { Ausprägung } \\
3\end{array}$}} & \multirow{2}{*}{\multicolumn{2}{|c|}{$\begin{array}{l}\text { Ausprägung } \\
4\end{array}$}} & \multirow{2}{*}{\multicolumn{2}{|c|}{ Fehlende Werte }} & \multirow{3}{*}{$\begin{array}{l}\text { Gesam } \\
\mathrm{N} \\
\end{array}$} \\
\hline & & & & & & & & & & & \\
\hline & $\mathrm{N}$ & $\%$ & $\mathrm{~N}$ & $\%$ & $\mathrm{~N}$ & $\%$ & & & $\mathrm{~N}$ & $\%$ & \\
\hline b_w_gr1 & 55 & 13.29 & 9 & 2.17 & 7 & 1.69 & 305 & 73.67 & 38 & 9.18 & 414 \\
\hline b_w_gr2 & 7 & 1.69 & 159 & 38.41 & 203 & 49.03 & 9 & 2.17 & 36 & 8.70 & 414 \\
\hline b_w_gr3 & 7 & 1.69 & 203 & 49.03 & 163 & 39.37 & 5 & 1.21 & 36 & 8.70 & 414 \\
\hline b_w_gr4 & 308 & 74.40 & 8 & 1.93 & 4 & .97 & 56 & 13.53 & 38 & 9.18 & 414 \\
\hline
\end{tabular}

\begin{tabular}{|c|c|c|c|c|c|c|c|c|c|c|c|}
\hline \multirow{3}{*}{$\begin{array}{l}\text { Itembezeichnung } \\
\text { MZP3 }\end{array}$} & \multirow{2}{*}{\multicolumn{2}{|c|}{$\begin{array}{l}\text { Ausprägung } \\
1\end{array}$}} & \multirow{2}{*}{\multicolumn{2}{|c|}{$\begin{array}{l}\text { Ausprägung } \\
2\end{array}$}} & \multirow{2}{*}{\multicolumn{2}{|c|}{$\begin{array}{l}\text { Ausprägung } \\
3\end{array}$}} & \multirow{2}{*}{\multicolumn{2}{|c|}{$\begin{array}{l}\text { Ausprägung } \\
4\end{array}$}} & \multicolumn{2}{|c|}{ Fehlende Werte } & \multirow{3}{*}{$\begin{array}{l}\text { Gesamt } \\
\mathrm{N} \\
\end{array}$} \\
\hline & & & & & & & & & \multirow[b]{2}{*}{$\mathrm{N}$} & \multirow[b]{2}{*}{$\%$} & \\
\hline & $\mathrm{N}$ & $\%$ & $\mathrm{~N}$ & $\%$ & $\mathrm{~N}$ & $\%$ & $\mathrm{~N}$ & $\%$ & & & \\
\hline c_w_gr1 & 35 & 10.17 & 8 & 2.33 & 1 & .29 & 278 & 80.81 & 22 & 6.40 & 344 \\
\hline c_w_gr2 & -- & -- & 148 & 43.02 & 172 & 50.00 & 3 & .87 & 21 & 6.10 & 344 \\
\hline c_w_gr3 & 5 & 1.45 & 159 & 46.22 & 150 & 43.60 & 9 & 2.61 & 21 & 6.10 & 344 \\
\hline c_w_gr4 & 285 & 82.84 & 7 & 2.03 & 1 & .29 & 31 & 9.01 & 20 & 5.81 & 344 \\
\hline
\end{tabular}




\section{$5 \quad$ Angaben zur Stichprobe ${ }^{7}$}

\subsection{Personenbezogene Angaben}

\subsubsection{Alter}

Kurzbezeichnung Variablenname

Instruktion

Skalierung (Antwortformat)

Instrument a_age/b_age/c_age

Wie alt bist du?

offen

MZP1: Hauptfragebogen
MZP2: Zusatzbogen Erstteilnahme
MZP3: Zusatzbogen Erstteilnahme

\begin{tabular}{|c|c|c|c|c|c|c|c|c|c|c|c|c|c|c|c|}
\hline & \multirow[t]{2}{*}{ Itembezeichnung } & \multicolumn{2}{|c|}{$\begin{array}{l}\text { Ausprägung } \\
16\end{array}$} & \multicolumn{2}{|c|}{$\begin{array}{l}\text { Ausprägung } \\
17\end{array}$} & \multicolumn{2}{|c|}{$\begin{array}{l}\text { Ausprägung } \\
18\end{array}$} & \multicolumn{2}{|c|}{$\begin{array}{l}\text { Ausprägung } \\
19\end{array}$} & \multicolumn{2}{|c|}{$\begin{array}{l}\text { Ausprägung } \\
20\end{array}$} & \multicolumn{2}{|c|}{$\begin{array}{l}\text { Ausprägung } \\
21\end{array}$} & \multicolumn{2}{|c|}{$\begin{array}{l}\text { Ausprägung } \\
22\end{array}$} \\
\hline & & $\mathrm{N}$ & $\%$ & $\mathrm{~N}$ & $\%$ & $\mathrm{~N}$ & $\%$ & $\mathrm{~N}$ & $\%$ & $\mathrm{~N}$ & $\%$ & $\mathrm{~N}$ & $\%$ & $\mathrm{~N}$ & $\%$ \\
\hline MZP1 & a_age & 20 & 4.49 & 55 & 12.56 & 158 & 35.51 & 108 & 24.27 & 59 & 13.26 & 14 & 3.15 & 9 & 2.02 \\
\hline MZP2 & b_age & 1 & 1.72 & 10 & 17.24 & 9 & 15.52 & 16 & 27.59 & 10 & 17.24 & 3 & 5.17 & 3 & 5.17 \\
\hline MZP3 & c_age & -- & -- & 2 & 6.06 & 7 & 21.21 & 17 & 51.52 & 1 & 3.03 & 3 & 9.09 & 2 & 6.06 \\
\hline Gesamt & & 21 & 3.92 & 67 & 12.50 & 174 & 32.46 & 141 & 26.31 & 70 & 13.06 & 20 & 3.73 & 14 & 2.61 \\
\hline
\end{tabular}

${ }_{7}$ Die in diesem Kapitel dargestellten demographischen Angaben (wie Alter und Geschlecht) sowie Angaben zu den Eingangsvoraussetzungen (wie Vorerfahrungen, Motivation) basieren auf Items, die zum ersten Messzeitpunkt in den Hauptfragebogen integriert waren. Zum zweiten und dritten Messzeitpunkt wurden diese Daten nur noch von denjenigen, die zu den jeweiligen Zeitpunkten erstmalig teilnahmen, erfasst, indem sie gebeten wurden, einen entsprechenden Zusatzbogen auszufüllen. 


\begin{tabular}{|c|c|c|c|c|c|c|c|c|c|c|c|c|c|c|c|c|}
\hline & \multirow[t]{2}{*}{ Itembezeichnung } & \multicolumn{2}{|c|}{$\begin{array}{l}\text { Ausprägung } \\
23\end{array}$} & \multicolumn{2}{|c|}{$\begin{array}{l}\text { Ausprägung } \\
24\end{array}$} & \multicolumn{2}{|c|}{$\begin{array}{l}\text { Ausprägung } \\
25\end{array}$} & \multicolumn{2}{|c|}{$\begin{array}{l}\text { Ausprägung } \\
26\end{array}$} & \multicolumn{2}{|c|}{$\begin{array}{l}\text { Ausprägung } \\
27\end{array}$} & \multicolumn{2}{|c|}{ Fehlende Werte } & \multicolumn{3}{|c|}{ Gesamt } \\
\hline & & $\mathrm{N}$ & $\%$ & $\mathrm{~N}$ & $\%$ & $\mathrm{~N}$ & $\%$ & $\mathrm{~N}$ & $\%$ & $\mathrm{~N}$ & $\%$ & $\mathrm{~N}$ & $\%$ & $\mathrm{~N}$ & MW & $\mathrm{SD}$ \\
\hline MZP1 & a_age & 4 & .90 & 4 & .90 & -- & -- & -- & -- & -- & -- & 14 & 3.15 & 445 & 18.59 & 1.4 \\
\hline MZP2 & b_age & 1 & 1.72 & -- & -- & 1 & 1.72 & 1 & 1.72 & 1 & 1.72 & 2 & 3.45 & 58 & 19.32 & 2.20 \\
\hline \multirow[t]{2}{*}{ MZP3 } & c_age & -- & -- & -- & -- & 1 & 3.03 & -- & -- & -- & -- & -- & -- & 33 & 19.24 & 1.58 \\
\hline & Gesamt & 4 & .93 & 4 & .75 & 2 & .37 & 1 & .91 & 1 & .19 & 16 & 2.99 & 536 & 18.71 & 1.54 \\
\hline
\end{tabular}




\subsubsection{Geschlecht}

Kurzbezeichnung Variablenname

Instruktion

Skalierung (Antwortformat)

Instrument a_sex/b_sex/c ssex

Welches Geschlecht hast du?

$1=$ männlich

$2=$ weiblich

$3=$ keine Zuordnung

MZP1: Hauptfragebogen
MZP2: Zusatzbogen Erstteilnahme
MZP3: Zusatzbogen Erstteilnahme

\begin{tabular}{|c|c|c|c|c|c|c|c|c|c|c|}
\hline & \multirow[t]{3}{*}{ Itembezeichnung } & \multirow{2}{*}{\multicolumn{2}{|c|}{$\begin{array}{l}\text { Ausprägung } \\
1\end{array}$}} & \multirow{2}{*}{\multicolumn{2}{|c|}{$\begin{array}{l}\text { Ausprägung } \\
2\end{array}$}} & \multirow{2}{*}{\multicolumn{2}{|c|}{$\begin{array}{l}\text { Ausprägung } \\
3\end{array}$}} & \multirow{2}{*}{\multicolumn{2}{|c|}{ Fehlende Werte }} & \multirow[t]{2}{*}{ Gesamt } \\
\hline & & & & & & & & & & \\
\hline & & $\mathrm{N}$ & $\%$ & $\mathrm{~N}$ & $\%$ & $\mathrm{~N}$ & $\%$ & $\mathrm{~N}$ & $\%$ & $\mathrm{~N}$ \\
\hline MZP1 & a_sex & 151 & 33.93 & 275 & 61.80 & 5 & 1.12 & 14 & 3.15 & 445 \\
\hline MZP2 & b_sex & 22 & 37.93 & 35 & 60.34 & -- & -- & 1 & 1.72 & 58 \\
\hline \multirow[t]{2}{*}{ MZP3 } & c_sex & 11 & 33.33 & 22 & 66.67 & -- & -- & -- & -- & 33 \\
\hline & Gesamt & 184 & 34.33 & 332 & 61.94 & 5 & .93 & 15 & 2.80 & 536 \\
\hline
\end{tabular}




\subsubsection{Schulabschluss}

Kurzbezeichnung Variablenname

Instruktion

Skalierung (Antwortformat)

Instrument $a \_a b / b \_a b / c \_a b$

Welches ist dein höchster Schulabschluss? Kreuze bitte eine Antwort an.

$1=$ Abitur oder Fachabitur

$2=$ Berufsgrundbildungsjahr/Berufsschule/Berufsfachschule

$3=$ Mittlere Reife / Realschulabschluss

$4=$ Hauptschulabschluss

$5=$ Abschluss einer Sonderschule/ Förderschule

$6=$ Sonstiger Schulabschluss (z. B. im Ausland)

$7=$ Ich bin ohne Abschluss von der Schule abgegangen

MZP1: Hauptfragebogen

MZP2: Zusatzbogen Erstteilnahme

MZP3: Zusatzbogen Erstteilnahme

\begin{tabular}{|c|c|c|c|c|c|c|c|c|c|c|c|c|c|c|c|c|c|c|}
\hline & \multirow[t]{2}{*}{ Itembezeichnung } & \multicolumn{2}{|c|}{$\begin{array}{l}\text { Ausprägung } \\
1\end{array}$} & \multicolumn{2}{|c|}{$\begin{array}{l}\text { Ausprägung } \\
2\end{array}$} & \multicolumn{2}{|c|}{$\begin{array}{l}\text { Ausprägung } \\
3\end{array}$} & \multicolumn{2}{|c|}{$\begin{array}{l}\text { Ausprägung } \\
4\end{array}$} & \multicolumn{2}{|c|}{$\begin{array}{l}\text { Ausprägung } \\
5\end{array}$} & \multicolumn{2}{|c|}{$\begin{array}{l}\text { Ausprägung } \\
6\end{array}$} & \multicolumn{2}{|c|}{$\begin{array}{l}\text { Ausprägung } \\
7\end{array}$} & \multicolumn{2}{|c|}{$\begin{array}{l}\text { Fehlende } \\
\text { Werte }\end{array}$} & \multirow[t]{2}{*}{ Gesamt } \\
\hline & & $\mathrm{N}$ & $\%$ & $\mathrm{~N}$ & $\%$ & $\mathrm{~N}$ & $\%$ & $\mathrm{~N}$ & $\%$ & $\mathrm{~N}$ & $\%$ & $\mathrm{~N}$ & $\%$ & $\mathrm{~N}$ & $\%$ & $\mathrm{~N}$ & $\%$ & \\
\hline MZP1 & a_ab & 297 & 66.74 & 16 & 3.60 & 103 & 23.15 & 10 & 2.25 & -- & -- & 1 & .22 & 1 & .22 & 17 & 3.82 & 445 \\
\hline MZP2 & b_ab & 30 & 51.72 & 1 & 1.72 & 23 & 39.66 & 2 & 3.45 & -- & -- & 1 & 1.72 & -- & -- & 1 & 1.72 & 58 \\
\hline \multirow[t]{2}{*}{ MZP3 } & c_ab & 25 & 75.75 & 2 & 6.06 & 4 & 12.12 & -- & -- & -- & -- & 1 & 3.03 & -- & -- & 1 & 3.03 & 33 \\
\hline & Gesamt & 352 & 66.67 & 19 & 3.54 & 130 & 24.25 & 12 & 2.24 & -- & -- & 3 & .56 & 1 & .19 & 19 & 3.54 & 536 \\
\hline
\end{tabular}




\subsubsection{Besuchte Schulform}

Kurzbezeichnung Variablenname

Instruktion

Skalierung (Antwortformat)

Anzahl Items

Instrument a_schul/b_schul/c_schul

Auf was für einer Schule warst du? Wenn du mehrere Schulen besucht hast, kreuze bitte auch mehrere Schulen an.

Dichotom; $0=$ trifft nicht zu, $1=$ trifft $z u$

7

MZP1: Hauptfrage- $\quad$ MZP2: Zusatzbogen MZP3: Zusatzbogen bogen
Erstteilnahme

Erstteilnahme

\begin{tabular}{llll}
\hline MZP1 & MZP2 & MZP3 & Itemwortlaut \\
\hline a_schul1 & b_schul1 & c_schul1 & Hauptschule \\
a_schul2 & b_schul2 & c_schul2 & Realschule \\
a_schul3 & b_schul3 & c_schul3 & Gymnasium \\
a_schul4 & b_schul4 & c_schul4 & Förderschule \\
a_schul5 & b_schul5 & c_schul5 & Kooperierte Gesamtschule (KGS) \\
a_schul6 & b_schul6 & c_schul6 & Integrierte Gesamtschule (IGS) \\
a_schuls & b_schuls & c_schuls & Sonstige \\
\hline
\end{tabular}




\begin{tabular}{|c|c|c|c|c|c|c|c|c|c|c|c|c|c|c|c|c|c|c|c|c|}
\hline \multirow{2}{*}{\multicolumn{3}{|c|}{ Itembezeichnung }} & \multicolumn{6}{|c|}{ MZP1 $(\mathrm{N}=445)$} & \multicolumn{6}{|c|}{ MZP2 $(\mathrm{N}=58)$} & \multicolumn{6}{|c|}{ MZP3 (N = 33) } \\
\hline & & & \multirow{2}{*}{\multicolumn{2}{|c|}{$\begin{array}{l}\text { Ausprägung } \\
0\end{array}$}} & \multirow{2}{*}{\multicolumn{2}{|c|}{$\begin{array}{l}\text { Ausprägung } \\
1\end{array}$}} & \multirow{2}{*}{\multicolumn{2}{|c|}{$\begin{array}{l}\text { Fehlende } \\
\text { Werte }\end{array}$}} & \multirow{2}{*}{\multicolumn{2}{|c|}{$\begin{array}{l}\text { Ausprägung } \\
0\end{array}$}} & \multirow{2}{*}{\multicolumn{2}{|c|}{$\begin{array}{l}\text { Ausprägung } \\
1\end{array}$}} & \multirow{2}{*}{\multicolumn{2}{|c|}{$\begin{array}{l}\text { Fehlende } \\
\text { Werte }\end{array}$}} & \multirow{2}{*}{\multicolumn{2}{|c|}{$\begin{array}{l}\text { Ausprägung } \\
0\end{array}$}} & \multirow{2}{*}{\multicolumn{2}{|c|}{$\begin{array}{l}\text { Ausprägung } \\
1\end{array}$}} & \multirow{2}{*}{\multicolumn{2}{|c|}{$\begin{array}{l}\text { Fehlende } \\
\text { Werte }\end{array}$}} \\
\hline \multirow[t]{2}{*}{ MZP1 } & \multirow[t]{2}{*}{ MZP2 } & \multirow[t]{2}{*}{ MZP3 } & & & & & & & & & & & & & & & & & & \\
\hline & & & $\mathrm{N}$ & $\%$ & $\mathrm{~N}$ & $\%$ & $\mathrm{~N}$ & $\%$ & $\mathrm{~N}$ & $\%$ & $\mathrm{~N}$ & $\%$ & $\mathrm{~N}$ & $\%$ & $\mathrm{~N}$ & $\%$ & $\mathrm{~N}$ & $\%$ & $\mathrm{~N}$ & $\%$ \\
\hline a_schul1 & b_schul1 & c_schul1 & 409 & 91.91 & 22 & 4.94 & 14 & 3.15 & 48 & 82.76 & 6 & 10.34 & 4 & 6.90 & 31 & 93.94 & 1 & 3.03 & 1 & 3.03 \\
\hline a_schul2 & b_schul2 & c_schul2 & 318 & 71.46 & 113 & 25.39 & 14 & 3.15 & 35 & 60.34 & 19 & 32.76 & 4 & 6.90 & 25 & 75.76 & 7 & 21.21 & 1 & 3.03 \\
\hline a_schul3 & b_schul3 & c_schul3 & 179 & 40.22 & 252 & 56.63 & 14 & 3.15 & 26 & 44.83 & 28 & 48.28 & 4 & 6.90 & 14 & 42.42 & 18 & 54.55 & 1 & 3.03 \\
\hline a_schul4 & b_schul4 & c_schul4 & 430 & 96.63 & 1 & .22 & 14 & 3.15 & 54 & 93.10 & -- & -- & 4 & 6.90 & 31 & 93.94 & 1 & 3.03 & 1 & 3.03 \\
\hline a_schul5 & b_schul5 & c_schul5 & 372 & 83.60 & 59 & 13.25 & 14 & 3.15 & 48 & 82.76 & 6 & 10.34 & 4 & 6.90 & 26 & 78.79 & 6 & 18.18 & 1 & 3.03 \\
\hline a_schul6 & b_schul6 & c_schul6 & 398 & 89.44 & 33 & 7.41 & 14 & 3.15 & 48 & 82.76 & 6 & 10.34 & 4 & 6.90 & 31 & 93.94 & 1 & 3.03 & 1 & 3.03 \\
\hline a_schuls & b_schuls & C_schuls & 354 & 79.55 & 77 & 17.30 & 14 & 3.15 & 48 & 82.76 & 6 & 10.34 & 4 & 6.90 & 29 & 87.89 & 3 & 9.09 & 1 & 3.03 \\
\hline
\end{tabular}

\begin{tabular}{|c|c|c|c|c|c|c|}
\hline \multirow[t]{4}{*}{ Itembezeichnung } & \multicolumn{6}{|c|}{ Gesamt $(\mathrm{N}=536)$} \\
\hline & \multicolumn{2}{|c|}{ Ausprägung } & \multicolumn{2}{|c|}{ Ausprägung } & \multicolumn{2}{|c|}{ Fehlende Werte } \\
\hline & 0 & & 1 & & & \\
\hline & $\mathrm{N}$ & $\%$ & $\mathrm{~N}$ & $\%$ & $\mathrm{~N}$ & $\%$ \\
\hline schul1 & 488 & 91.05 & 29 & 5.41 & 19 & 3.55 \\
\hline schul2 & 378 & 70.52 & 139 & 25.93 & 19 & 3.55 \\
\hline schul3 & 219 & 40.86 & 298 & 55.60 & 19 & 3.55 \\
\hline schul4 & 515 & 96.08 & 2 & .37 & 19 & 3.55 \\
\hline schul5 & 446 & 83.21 & 71 & 13.25 & 19 & 3.55 \\
\hline schul6 & 477 & 88.99 & 40 & 7.46 & 19 & 3.55 \\
\hline schuls & 431 & 80.41 & 86 & 16.05 & 19 & 3.55 \\
\hline
\end{tabular}




\subsection{Rahmendaten zum Freiwilligendienst}

\subsection{1 $\mathrm{FSJ} / \mathrm{BFD}$}

Kurzbezeichnung Variablenname

Instruktion

Skalierung (Antwortformat)

Instrument a_fd/b_fd/c_fd

Absolvierst du ein Freiwilliges Soziales Jahr (FSJ) oder einen Bundesfreiwilligendienst (BFD)?

$1=\mathrm{FSJ}$

$2=\mathrm{BFD}$

MZP1: Hauptfrage-

bogen

MZP2: Zusatzbogen

Erstteilnahme

MZP3: Zusatzbogen

Erstteilnahme

\begin{tabular}{|c|c|c|c|c|c|c|c|c|}
\hline & \multirow[t]{3}{*}{ Itembezeichnung } & \multirow{2}{*}{\multicolumn{2}{|c|}{$\begin{array}{l}\text { Ausprägung } \\
1\end{array}$}} & \multirow{2}{*}{\multicolumn{2}{|c|}{$\begin{array}{l}\text { Ausprägung } \\
2\end{array}$}} & \multicolumn{2}{|c|}{ Fehlende Werte } & \multirow{3}{*}{$\begin{array}{l}\text { Gesamt } \\
\mathrm{N}\end{array}$} \\
\hline & & & & & & & & \\
\hline & & $\mathrm{N}$ & $\%$ & $\mathrm{~N}$ & $\%$ & $\mathrm{~N}$ & $\%$ & \\
\hline MZP1 & a_fd & 430 & 96.63 & 15 & 3.37 & -- & -- & 445 \\
\hline MZP2 & b_fd & 55 & 94.83 & 3 & 5.17 & -- & -- & 58 \\
\hline \multirow[t]{2}{*}{ MZP3 } & c_fd & 32 & 96.96 & 1 & 3.03 & -- & -- & 33 \\
\hline & Gesamt & 517 & 96.46 & 19 & 3.54 & -- & -- & 536 \\
\hline
\end{tabular}




\subsubsection{Einsatzstellenbereich}

Kurzbezeichnung Variablenname

Instruktion

Skalierung (Antwortformat)

Instrument a_fd_b/b_fd_b/c_fd_b

Machst du ein FSJ/ BFD im Bereich Menschen mit Behinderung / psychischer Erkrankung? [Filterfrage]

$0=$ Nein $\rightarrow$ weiter mit Frage 4

$1=\mathrm{Ja} \rightarrow$ weiter mit Frage 3

MZP1: Hauptfrage- MZP2: Zusatzbogen MZP3: Zusatzbogen bogen Erstteilnahme Erstteilnahme

\begin{tabular}{|c|c|c|c|c|c|c|c|}
\hline \multirow[t]{3}{*}{ Itembezeichnung } & \multirow{2}{*}{\multicolumn{2}{|c|}{$\begin{array}{l}\text { Ausprägung } \\
0\end{array}$}} & \multirow{2}{*}{\multicolumn{2}{|c|}{$\begin{array}{l}\text { Ausprägung } \\
1\end{array}$}} & \multicolumn{2}{|c|}{ Fehlende Werte } & \multirow[t]{2}{*}{ Gesam } \\
\hline & & & & & & & \\
\hline & $\mathrm{N}$ & $\%$ & $\mathrm{~N}$ & $\%$ & $\mathrm{~N}$ & $\%$ & $\mathrm{~N}$ \\
\hline a_fd_b & 162 & 36.40 & 281 & 63.15 & 2 & .45 & 445 \\
\hline b_fd_b & 26 & 44.83 & 31 & 53.45 & 1 & 1.72 & 58 \\
\hline c_fd_b & 5 & 15.15 & 28 & 84.85 & -- & -- & 33 \\
\hline Gesamt & 193 & 36.01 & 340 & 63.43 & 3 & .56 & 536 \\
\hline
\end{tabular}




\subsubsection{Einsatzstellen für oder mit Menschen mit Behinderung}
Kurzbezeichnung Variablenname
a_esb/b_esb/c_esb
Instruktion
In was für einer Einrichtung für Menschen mit Behinderung oder psychischer Erkrankung machst du den FSJ/BFD?
Skalierung (Antwortformat)
Dichotom; $0=$ trifft nicht $z u ; 1=$ trifft $z u$
Instrument
MZP1: Hauptfrage-
bogen
MZP2: Zusatzbogen
Erstteilnahme
MZP3: Zusatzbogen
Erstteilnahme

\begin{tabular}{llll}
\hline Itembezeichnung & & \\
MZP1 & MZP2 & MZP3 & Itemwortlaut \\
\hline a_esb1 & b_esb1 & c_esb1 & Werkstätten für Menschen mit Behinderung \\
a_esb2 & b_esb2 & c_esb2 & Werkstätten für Menschen mit psychischer Erkrankung \\
a_esb3 & b_esb3 & c_esb3 & Tagesförderung für Menschen mit Behinderung \\
a_esb4 & b_esb4 & c_esb4 & Wohngruppen für Menschen mit Behinderung \\
a_esb5 & b_esb5 & c_esb5 & Rehabilitation für Menschen mit Behinderung \\
a_esb6 & b_esb6 & c_esb6 & Förderschule (Grundschule) \\
a_esb7 & b_esb7 & c_esb7 & Förderschule (Sekundarstufe) \\
a_esb8 & b_esb8 & c_esb8 & Begleitung von Schüler/innen mit Behinderung in einer Regelschule \\
a_esb9 & b_esb9 & c_esb9 & Heilpädagogische Krippe oder Kita \\
a_esb10 & b_esb10 & c_esb10 & Integrative Kita \\
a_esb11 & b_esb11 & c_esb11 & Psychiatrie für Erwachsene \\
a_esb12 & b_esb12 & c_esb12 & Kinder- und Jugendpsychiatrie \\
a_esbs & b_esbs & c_esbs & Sonstiges \\
\hline
\end{tabular}




\begin{tabular}{|c|c|c|c|c|c|c|c|c|c|c|c|c|c|c|c|c|c|c|}
\hline \multicolumn{3}{|c|}{ Itembezeichnung } & \multicolumn{4}{|c|}{$\operatorname{MZP1}(\mathrm{N}=281)^{\mathrm{a}}$} & \multicolumn{4}{|c|}{$\operatorname{MZP} 2(N=31)^{a}$} & \multicolumn{4}{|c|}{$\mathrm{MZP} 3(\mathrm{~N}=28)^{\mathrm{a}}$} & \multicolumn{4}{|c|}{ Gesamt $(\mathrm{N}=340)^{\mathrm{a}}$} \\
\hline \multirow[b]{3}{*}{ MZP1 } & \multirow[b]{3}{*}{ MZP2 } & \multirow[b]{3}{*}{ MZP3 } & \multirow{2}{*}{\multicolumn{2}{|c|}{$\begin{array}{l}\text { Ausprägung } \\
0\end{array}$}} & \multirow{2}{*}{\multicolumn{2}{|c|}{$\begin{array}{l}\text { Ausprägung } \\
1\end{array}$}} & \multirow{2}{*}{\multicolumn{2}{|c|}{$\begin{array}{l}\text { Ausprägung } \\
0\end{array}$}} & \multirow{2}{*}{\multicolumn{2}{|c|}{$\begin{array}{l}\text { Ausprägung } \\
1\end{array}$}} & \multirow{2}{*}{\multicolumn{2}{|c|}{$\begin{array}{l}\text { Ausprägung } \\
0\end{array}$}} & \multirow{2}{*}{\multicolumn{2}{|c|}{$\begin{array}{l}\text { Ausprägung } \\
1\end{array}$}} & \multirow{2}{*}{\multicolumn{2}{|c|}{$\begin{array}{l}\text { Ausprägung } \\
0\end{array}$}} & \multirow{2}{*}{\multicolumn{2}{|c|}{$\begin{array}{l}\text { Ausprägung } \\
1\end{array}$}} \\
\hline & & & & & & & & & & & & & & & & & & \\
\hline & & & $\mathrm{N}$ & $\%$ & $\mathrm{~N}$ & $\%$ & $\mathrm{~N}$ & $\%$ & $\mathrm{~N}$ & $\%$ & $\mathrm{~N}$ & $\%$ & $\mathrm{~N}$ & $\%$ & $\mathrm{~N}$ & $\%$ & $\mathrm{~N}$ & $\%$ \\
\hline a_esb1 & b_esb1 & c_esb1 & 211 & 75.09 & 70 & 24.91 & 23 & 74.19 & 8 & 25.81 & 26 & 92.86 & 2 & 7.14 & 260 & 76.47 & 80 & 23.53 \\
\hline a_esb2 & b_esb2 & c_esb2 & 249 & 88.61 & 31 & 11.03 & 30 & 96.77 & 1 & 3.23 & 27 & 96.43 & 1 & 3.57 & 306 & 90.00 & 33 & 9.71 \\
\hline a_esb3 & b_esb3 & c_esb3 & 243 & 86.48 & 38 & 13.52 & 25 & 80.65 & 6 & 19.35 & 24 & 85.71 & 4 & 14.29 & 292 & 85.88 & 48 & 14.12 \\
\hline a_esb4 & b_esb4 & c_esb4 & 261 & 92.88 & 20 & 7.12 & 27 & 87.10 & 4 & 12.90 & 28 & 100 & -- & -- & 316 & 92.94 & 24 & 7.06 \\
\hline a_esb5 & b_esb5 & c_esb5 & 274 & 97.51 & 7 & 2.49 & 28 & 90.32 & 3 & 9.68 & 28 & 100 & -- & -- & 330 & 97.06 & 10 & 2.94 \\
\hline a_esb6 & b_esb6 & c_esb6 & 243 & 86.48 & 38 & 13.52 & 27 & 87.10 & 4 & 12.90 & 26 & 92.86 & 2 & 7.14 & 296 & 87.06 & 44 & 12.94 \\
\hline a_esb7 & b_esb7 & c_esb7 & 237 & 84.34 & 44 & 15.66 & 27 & 87.10 & 4 & 12.90 & 24 & 85.71 & 4 & 14.29 & 288 & 84.71 & 52 & 15.29 \\
\hline a_esb8 & b_esb8 & c_esb8 & 251 & 89.32 & 30 & 10.68 & 27 & 87.10 & 4 & 12.90 & 15 & 53.57 & 13 & 46.43 & 293 & 86.18 & 47 & 13.82 \\
\hline a_esb9 & b_esb9 & c_esb9 & 254 & 90.39 & 27 & 9.61 & 31 & 100 & -- & -- & 26 & 92.86 & 2 & 7.14 & 311 & 91.47 & 29 & 8.53 \\
\hline a_esb10 & b_esb10 & c_esb10 & 261 & 92.88 & 20 & 7.12 & 27 & 87.10 & 4 & 12.90 & 28 & 100 & -- & -- & 316 & 92.94 & 24 & 7.06 \\
\hline a_esb11 & b_esb11 & c_esb11 & 268 & 95.37 & 13 & 4.63 & 30 & 96.77 & 1 & 3.23 & 27 & 96.43 & 1 & 3.57 & 325 & 95.59 & 15 & 4.41 \\
\hline a_esb12 & b_esb12 & c_esb12 & 273 & 97.15 & 8 & 2.85 & 31 & 100 & -- & -- & 28 & 100 & -- & -- & 332 & 97.65 & 8 & 2.35 \\
\hline a_esbs & b_esbs & c_esbs & 244 & 86.83 & 37 & 13.17 & 30 & 96.77 & 1 & 3.23 & 28 & 100 & -- & -- & 302 & 88.82 & 38 & 11.18 \\
\hline
\end{tabular}

${ }^{a} \mathrm{~N}=$ diejenigen, die bei vorheriger Filterfrage (siehe 5.2.2) angegeben haben, ein FSJ/BFD im Bereich Menschen mit Behinderung/psychischer Erkrankung zu absolvieren. 


\subsubsection{Andere Einsatzstellen}

\begin{tabular}{|c|c|c|c|c|c|c|}
\hline \multicolumn{4}{|c|}{ Kurzbezeichnung Variablenname } & \multicolumn{3}{|l|}{ a_esa/b_esa/c_esa } \\
\hline \multicolumn{4}{|c|}{ Instruktion } & \multicolumn{3}{|c|}{ In was für einer Einrichtung machst du den FSJ/BFD? } \\
\hline \multicolumn{4}{|c|}{ Skalierung (Antwortformat) } & \multicolumn{3}{|c|}{ Dichotom; $0=$ trifft nicht zu, $1=$ trifft $z u$} \\
\hline \multicolumn{4}{|c|}{ Instrument } & $\begin{array}{l}\text { MZP1: Hauptfrage- } \\
\text { bogen }\end{array}$ & $\begin{array}{l}\text { MZP2: Zusatzbogen } \\
\text { Erstteilnahme }\end{array}$ & $\begin{array}{l}\text { MZP3: Zusatzbogen } \\
\text { Erstteilnahme }\end{array}$ \\
\hline \multicolumn{7}{|c|}{ Itembezeichnung } \\
\hline MZP1 & MZP2 & MZP3 & \multicolumn{4}{|c|}{ Itemwortlaut } \\
\hline a_esa1 & b_esa1 & c_esa1 & \multicolumn{4}{|c|}{ Altenpflege } \\
\hline a_esa2 & b_esa2 & c_esa2 & \multicolumn{4}{|c|}{ Krippe oder Kita } \\
\hline a_esa3 & b_esa3 & c_esa3 & \multicolumn{4}{|c|}{ Krankenhaus } \\
\hline a_esa4 & b_esa4 & c_esa4 & \multicolumn{4}{|c|}{ Jugendzentrum } \\
\hline a_esas & b_esas & c_esas & \multicolumn{4}{|c|}{ Sonstiges } \\
\hline
\end{tabular}




\begin{tabular}{|c|c|c|c|c|c|c|c|c|c|c|c|c|c|c|c|c|c|c|}
\hline \multicolumn{3}{|c|}{ Itembezeichnung } & \multicolumn{4}{|c|}{ MZP1 $(\mathrm{N}=162)^{a}$} & \multicolumn{4}{|c|}{$\operatorname{MZP2}(\mathrm{N}=26)^{\mathrm{a}}$} & \multicolumn{4}{|c|}{ MZP3 $(\mathrm{N}=5)^{\mathrm{a}}$} & \multicolumn{4}{|c|}{ Gesamt $(\mathbf{N}=193)^{a}$} \\
\hline & & & \multirow{2}{*}{\multicolumn{2}{|c|}{$\begin{array}{l}\text { Ausprägung } \\
0\end{array}$}} & \multirow{2}{*}{\multicolumn{2}{|c|}{$\begin{array}{l}\text { Ausprägung } \\
1\end{array}$}} & \multirow{2}{*}{\multicolumn{2}{|c|}{$\begin{array}{l}\text { Ausprägung } \\
0\end{array}$}} & \multirow{2}{*}{\multicolumn{2}{|c|}{$\begin{array}{l}\text { Ausprägung } \\
1\end{array}$}} & \multirow{2}{*}{\multicolumn{2}{|c|}{$\begin{array}{l}\text { Ausprägung } \\
0\end{array}$}} & \multirow{2}{*}{\multicolumn{2}{|c|}{$\begin{array}{l}\text { Ausprägung } \\
1\end{array}$}} & \multirow{2}{*}{\multicolumn{2}{|c|}{$\begin{array}{l}\text { Ausprägung } \\
0\end{array}$}} & \multirow{2}{*}{\multicolumn{2}{|c|}{$\begin{array}{l}\text { Ausprägung } \\
1\end{array}$}} \\
\hline & & & & & & & & & & & & & & & & & & \\
\hline MZP1 & MZP2 & MZP3 & $\mathrm{N}$ & $\%$ & $\mathrm{~N}$ & $\%$ & $\mathrm{~N}$ & $\%$ & $\mathrm{~N}$ & $\%$ & $\mathrm{~N}$ & $\%$ & $\mathrm{~N}$ & $\%$ & $\mathrm{~N}$ & $\%$ & $\mathrm{~N}$ & $\%$ \\
\hline a_esa1 & b_esa1 & c_esa1 & 156 & 96.30 & 6 & 3.70 & 23 & 88.46 & 3 & 11.54 & 4 & 80.00 & 1 & 20.00 & 183 & 94.82 & 10 & 5.18 \\
\hline a_esa2 & b_esa2 & c_esa2 & 110 & 67.90 & 52 & 32.10 & 9 & 34.62 & 17 & 65.38 & 2 & 40.00 & 3 & 60.00 & 121 & 62.69 & 72 & 37.31 \\
\hline a_esa3 & b_esa3 & c_esa3 & 118 & 72.84 & 44 & 27.16 & 21 & 80.77 & 5 & 19.23 & 3 & 60.00 & 2 & 40.00 & 142 & 73.58 & 51 & 26.42 \\
\hline a_esa4 & b_esa4 & c_esa4 & 158 & 97.53 & 4 & 2.47 & 26 & 100 & -- & -- & 5 & 100 & -- & -- & 189 & 97.93 & 4 & 2.07 \\
\hline a_esas & b_esas & c_esas & 104 & 64.20 & 58 & 35.80 & 24 & 92.31 & 2 & 7.69 & 4 & 80.00 & 1 & 20.00 & 132 & 68.39 & 61 & 31.61 \\
\hline
\end{tabular}

a $\mathrm{N}=$ diejenigen, die bei vorheriger Filterfrage (siehe 5.2.2) angegeben haben, kein FSJ/BFD im Bereich Menschen mit Behinderung/psychischer Erkrankung zu absolvieren 


\subsubsection{Arbeitsbeginn}

Kurzbezeichnung Variablenname

Instruktion

Skalierung (Antwortformat)

Instrument a_begin/b_begin/c_begin

Wann war dein erster Arbeitstag in der Einsatzstelle?

$1=$ Vor etwa einer Woche

$2=$ Vor etwa zwei Wochen

$3=$ Vor drei oder noch mehr Wochen

$4=$ Ich habe bislang noch nicht in der Einsatzstelle gearbeitet

MZP1: Hauptfrage- $\quad$ MZP2: Zusatzbogen MZP3: Zusatzbogen bogen
Erstteilnahme
Erstteilnahme

\begin{tabular}{|c|c|c|c|c|c|c|c|c|c|c|c|}
\hline \multirow[t]{3}{*}{ Itembezeichnung } & \multirow{2}{*}{\multicolumn{2}{|c|}{$\begin{array}{l}\text { Ausprägung } \\
1\end{array}$}} & \multirow{2}{*}{\multicolumn{2}{|c|}{$\begin{array}{l}\text { Ausprägung } \\
2\end{array}$}} & \multirow{2}{*}{\multicolumn{2}{|c|}{$\begin{array}{l}\text { Ausprägung } \\
3\end{array}$}} & \multirow{2}{*}{\multicolumn{2}{|c|}{$\begin{array}{l}\text { Ausprägung } \\
4\end{array}$}} & \multirow{2}{*}{\multicolumn{2}{|c|}{$\begin{array}{l}\text { Fehlende } \\
\text { Werte }\end{array}$}} & \multirow{3}{*}{$\begin{array}{l}\text { Gesamt } \\
\mathrm{N}\end{array}$} \\
\hline & & & & & & & & & & & \\
\hline & $\mathrm{N}$ & $\%$ & $\mathrm{~N}$ & $\%$ & $\mathrm{~N}$ & $\%$ & $\mathrm{~N}$ & $\%$ & $\mathrm{~N}$ & $\%$ & \\
\hline a_begin & 51 & 11.46 & 28 & 6.29 & 344 & 77.30 & 19 & 4.27 & 3 & .67 & 445 \\
\hline b_begin & -- & -- & 3 & 5.17 & 51 & 87.93 & 2 & 3.45 & 2 & 3.45 & 58 \\
\hline c_begin & -- & -- & -- & -- & 29 & 87.88 & -- & -- & 4 & 12.12 & 33 \\
\hline Gesamt & 51 & 9.51 & 31 & 5.78 & 424 & 79.10 & 21 & 3.92 & 9 & 1.31 & 536 \\
\hline
\end{tabular}





\subsection{Individuelle Eingangsvoraussetzungen und Kontextvariab- len im Freiwilligendienst}

\subsubsection{Motivation zum Freiwilligendienst}

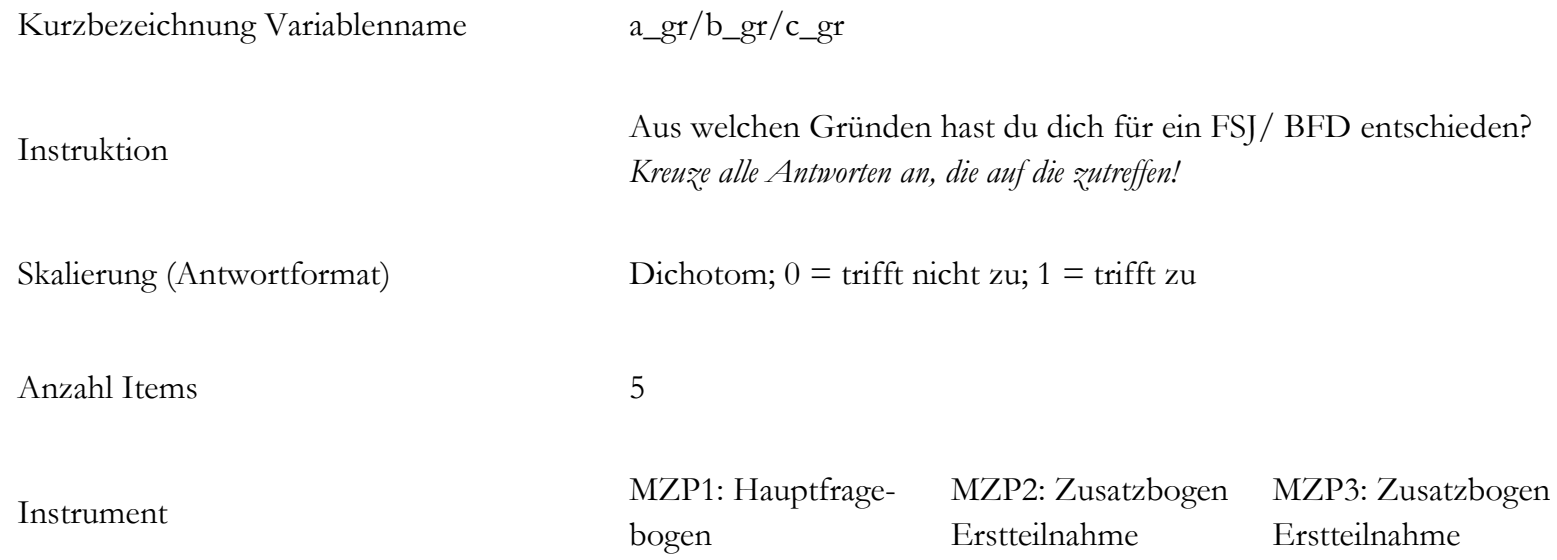

\begin{tabular}{llll}
\hline \multicolumn{2}{l}{ Itembezeichnung } & & \\
MZP1 & MZP2 & MZP3 & Itemwortlaut \\
\hline a_gr1 & b_gr1 & c_gr1 & Empfehlung von anderen (z. B. Freunden, Lehrer/innen, Familien) \\
a_gr2 & b_gr2 & c_gr2 & Berufsorientierung \\
a_gr3 & b_gr3 & c_gr3 & Um etwas Soziales zu machen \\
a_gr4 & b_gr4 & c_gr4 & Chancen auf Ausbildungs- oder Studienplatz verbessern \\
a_grs & b_grs & c_grs & Sonstiges \\
\hline
\end{tabular}




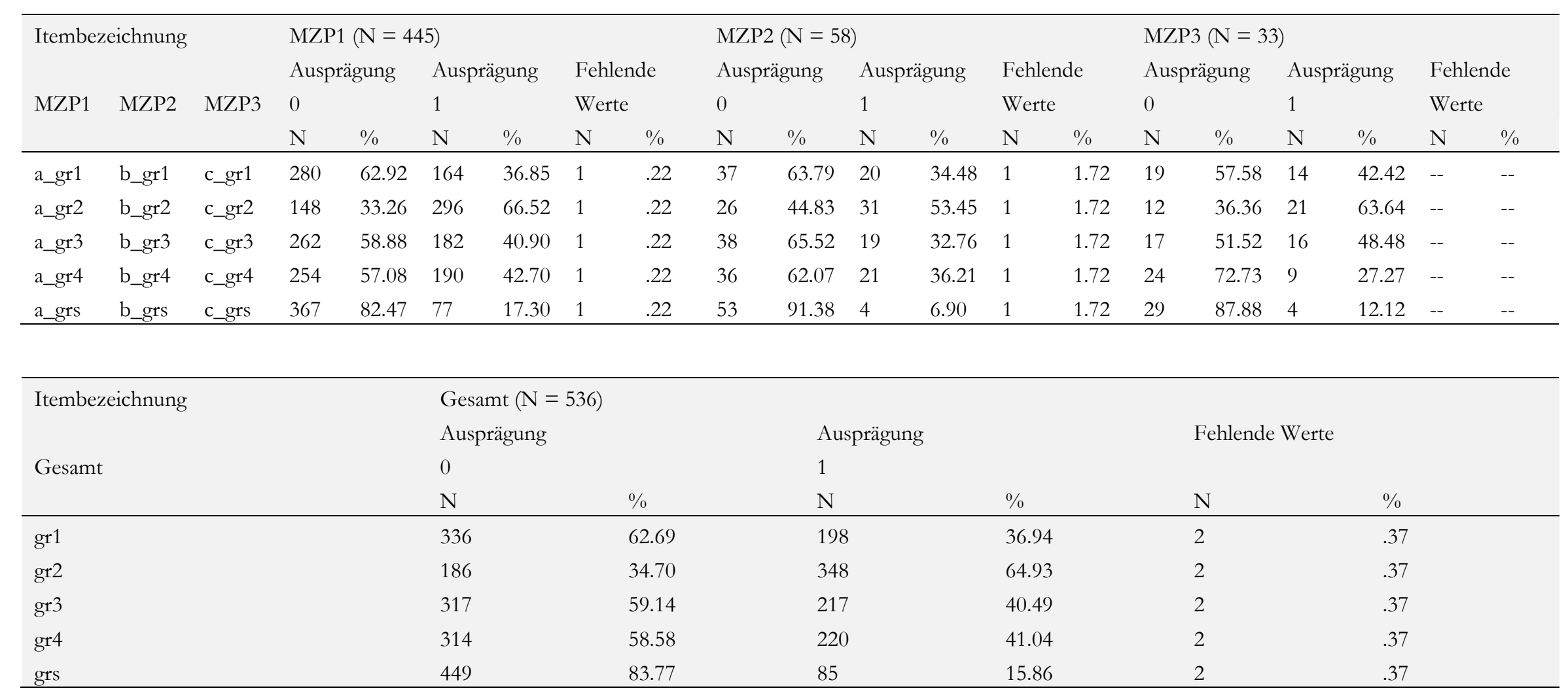




\subsubsection{Gründe für Einsatzstellenwahl}

Kurzbezeichnung Variablenname

Instruktion

Skalierung (Antwortformat)

Anzahl Items

Instrument a_gres/b_gres/c_gres

Aus welchen Gründen hast du dich für ein FSJ/ BFD in genau der Einsatzstelle entschieden? Kreuze alle Antworten an, die auf die zutreffen!

Dichotom; 0 = trifft nicht $z u, 1=$ trifft $z u$

7 bogen
MZP2: Zusatzbogen

Erstteilnahme
MZP3: Zusatzbogen

Erstteilnahme

\begin{tabular}{llll}
\hline MZP1 & MZP2 & MZP2 & Itemwortlaut \\
\hline a_gres1 & b_gres1 & c_gres1 & Empfehlung oder Zuteilung vom Träger \\
a_gres2 & b_gres2 & c_gres2 & Interesse an genau dem Bereich \\
a_gres3 & b_gres3 & c_gres3 & Privater Kontakt zur Einsatzstelle \\
a_gres4 & b_gres4 & c_gres4 & Private Vorerfahrungen in genau dem Bereich \\
a_gres5 & b_gres5 & c_gres5 & Sonstige Vorerfahrungen in genau dem Bereich \\
a_gres6 & b_gres6 & c_gres6 & Weil ich später in dem Bereich arbeiten möchte \\
a_gress & b_gress & c_gress & Sonstiges \\
\hline
\end{tabular}




\begin{tabular}{|c|c|c|c|c|c|c|c|c|c|c|c|c|c|c|c|c|c|c|c|c|}
\hline \multicolumn{3}{|c|}{ Itembezeichnung } & \multicolumn{6}{|c|}{ MZP3 $(\mathrm{N}=445)$} & \multicolumn{6}{|c|}{ MZP2 $(\mathrm{N}=58)$} & \multicolumn{6}{|c|}{ MZP3 $(\mathrm{N}=33)$} \\
\hline \multirow{3}{*}{ MZP1 } & \multirow{3}{*}{ MZP2 } & \multirow{3}{*}{ MZP3 } & \multirow{2}{*}{\multicolumn{2}{|c|}{$\begin{array}{l}\text { Ausprägung } \\
0\end{array}$}} & \multirow{2}{*}{\multicolumn{2}{|c|}{$\begin{array}{l}\text { Ausprägung } \\
1\end{array}$}} & \multirow{2}{*}{\multicolumn{2}{|c|}{$\begin{array}{l}\text { Fehlende } \\
\text { Werte }\end{array}$}} & \multirow{2}{*}{\multicolumn{2}{|c|}{$\begin{array}{l}\text { Ausprägung } \\
0\end{array}$}} & \multirow{2}{*}{\multicolumn{2}{|c|}{$\begin{array}{l}\text { Ausprägung } \\
1\end{array}$}} & \multirow{2}{*}{\multicolumn{2}{|c|}{$\begin{array}{l}\text { Fehlende } \\
\text { Werte }\end{array}$}} & \multirow{2}{*}{\multicolumn{2}{|c|}{$\begin{array}{l}\text { Ausprägung } \\
0\end{array}$}} & \multirow{2}{*}{\multicolumn{2}{|c|}{$\begin{array}{l}\text { Ausprägung } \\
1\end{array}$}} & \multirow{2}{*}{\multicolumn{2}{|c|}{$\begin{array}{l}\text { Fehlende } \\
\text { Werte }\end{array}$}} \\
\hline & & & & & & & & & & & & & & & & & & & & \\
\hline & & & $\mathrm{N}$ & $\%$ & $\mathrm{~N}$ & $\%$ & $\mathrm{~N}$ & $\%$ & $\mathrm{~N}$ & $\%$ & $\mathrm{~N}$ & $\%$ & $\mathrm{~N}$ & $\%$ & $\mathrm{~N}$ & $\%$ & $\mathrm{~N}$ & $\%$ & $\mathrm{~N}$ & $\%$ \\
\hline a_gres1 & b_gres1 & c_gres1 & 323 & 72.58 & 121 & 27.19 & 1 & .22 & 37 & 63.79 & 20 & 34.48 & 1 & 1.72 & 19 & 57.58 & 14 & 42.42 & $\begin{array}{ll}- \\
-\end{array}$ & -- \\
\hline a_gres2 & b_gres2 & c_gres2 & 204 & 45.84 & 240 & 53.93 & 1 & .22 & 33 & 56.90 & 24 & 41.38 & 1 & 1.72 & 21 & 63.64 & 12 & 36.36 & -- & -- \\
\hline a_gres3 & b_gres3 & c_gres3 & 361 & 81.12 & 83 & 18.65 & 1 & .22 & 49 & 84.48 & 8 & 13.79 & 1 & 1.72 & 28 & 84.85 & 5 & 15.15 & -- & -- \\
\hline a_gres4 & b_gres 4 & c_gres 4 & 389 & 87.42 & 55 & 12.36 & 1 & .22 & 53 & 91.38 & 4 & 6.90 & 1 & 1.72 & 32 & 96.97 & 1 & 3.03 & -- & -- \\
\hline a_gres5 & b_gres5 & c_gres 5 & 400 & 89.89 & 44 & 9.89 & 1 & .22 & 51 & 87.93 & 6 & 10.34 & 1 & 1.72 & 27 & 81.82 & 6 & 18.18 & -- & -- \\
\hline a_gres6 & b_gres6 & c_gres6 & 328 & 73.71 & 116 & 26.07 & 1 & .22 & 50 & 86.21 & 7 & 12.07 & 1 & 1.72 & 29 & 87.88 & 4 & 12.12 & -- & -- \\
\hline a_gress & b_gress & c_gress & 395 & 88.76 & 49 & 11.01 & 1 & .22 & 54 & 93.10 & 3 & 5.17 & 1 & 1.72 & 32 & 96.97 & 1 & 3.03 & -- & -- \\
\hline
\end{tabular}

\begin{tabular}{|c|c|c|c|c|c|c|}
\hline \multirow{4}{*}{$\begin{array}{l}\text { Itembezeichnung } \\
\text { Gesamt }\end{array}$} & \multicolumn{6}{|c|}{ Gesamt $(\mathrm{N}=536)$} \\
\hline & \multirow{2}{*}{\multicolumn{2}{|c|}{$\begin{array}{l}\text { Ausprägung } \\
0\end{array}$}} & \multirow{2}{*}{\multicolumn{2}{|c|}{$\begin{array}{l}\text { Ausprägung } \\
1\end{array}$}} & \multicolumn{2}{|c|}{ Fehlende Werte } \\
\hline & & & & & & \\
\hline & $\mathrm{N}$ & $\%$ & $\mathrm{~N}$ & $\%$ & $\mathrm{~N}$ & $\%$ \\
\hline gres1 & 379 & 70.71 & 155 & 28.92 & 2 & .37 \\
\hline gres2 & 258 & 48.13 & 276 & 51.49 & 2 & .37 \\
\hline gres3 & 438 & 81.72 & 96 & 17.91 & 2 & .37 \\
\hline gres4 & 474 & 88.43 & 60 & 11.19 & 2 & .37 \\
\hline gres5 & 478 & 89.18 & 56 & 10.45 & 2 & .37 \\
\hline gres6 & 407 & 75.93 & 127 & 23.69 & 2 & .37 \\
\hline gress & 481 & 89.74 & 53 & 9.89 & 2 & .37 \\
\hline
\end{tabular}




\subsubsection{Vorerfahrung mit Menschen mit Behinderung}

Kurzbezeichnung Variablenname

Instruktion

Skalierung (Antwortformat)

Anzahl Items

Instrument a_vor_b1/a_vor_b2/a_vor_b3/a_vor_b4/a_vor_bs/a_vor_b5

In welchem Bereich hast du vor deinem FSJ/BFD Erfahrungen mit Behinderung gemacht? Kreuze alle Antworten an, die auf die zutreffen!

Dichotom; $0=$ trifft nicht $z u, 1=$ trifft $z u$

6

MZP1: Hauptfrage-

bogen
MZP2: Zusatzbogen

Erstteilnahme
MZP3: Zusatzbogen Erstteilnahme

\begin{tabular}{|c|c|c|c|}
\hline MZP1 & MZP2 & MZP2 & Itemwortlaut \\
\hline a_vor_b1 & b_vor_b1 & c_vor_b1 & Behinderung oder psychischer Erkrankung war ein Thema im Unterricht \\
\hline a_vor_b2 & b_vor_b2 & c_vor_b2 & Ich hatte Mitschüler/innen mit Behinderung oder psychischer Erkrankung \\
\hline a_vor_b3 & b_vor_b3 & c_vor_b3 & $\begin{array}{l}\text { Ich habe schon mit Menschen mit Behinderung oder psychischer Erkran- } \\
\text { kung gearbeitet (im Nebenjob, Praktikum, Ehrenamt o. ä.) }\end{array}$ \\
\hline a_vor_b4 & b_vor_b4 & c_vor_b4 & $\begin{array}{l}\text { Ich habe im persönlichen Umfeld Menschen mit Behinderung oder psychi- } \\
\text { scher Erkrankung }\end{array}$ \\
\hline a_vor_bs & b_vor_bs & c_vor_bs & Sonstiges \\
\hline a_vor_b5 & b_vor_b5 & c_vor_b5 & $\begin{array}{l}\text { Ich habe vor meinem FSJ keine Erfahrungen mit Behinderung oder psychi- } \\
\text { schen Erkrankungen gemacht }\end{array}$ \\
\hline
\end{tabular}




\begin{tabular}{|c|c|c|c|c|c|c|c|c|c|c|c|c|c|c|c|c|c|c|c|c|}
\hline \multirow{2}{*}{\multicolumn{3}{|c|}{ Itembezeichnung }} & \multicolumn{6}{|c|}{ MZP1 (N=445) } & \multicolumn{6}{|c|}{ MZP2 (N= 58) } & \multicolumn{6}{|c|}{ MZP3 (N=33) } \\
\hline & & & \multirow{2}{*}{\multicolumn{2}{|c|}{$\begin{array}{l}\text { Ausprägung } \\
0\end{array}$}} & \multicolumn{2}{|c|}{ Ausprägung } & \multirow{2}{*}{\multicolumn{2}{|c|}{$\begin{array}{l}\text { Fehlende } \\
\text { Werte }\end{array}$}} & \multirow{2}{*}{\multicolumn{2}{|c|}{$\begin{array}{l}\text { Ausprägung } \\
0\end{array}$}} & \multirow{2}{*}{\multicolumn{2}{|c|}{$\begin{array}{l}\text { Ausprägung } \\
1\end{array}$}} & \multirow{2}{*}{\multicolumn{2}{|c|}{$\begin{array}{l}\text { Fehlende } \\
\text { Werte }\end{array}$}} & \multirow{2}{*}{\multicolumn{2}{|c|}{$\begin{array}{l}\text { Ausprägung } \\
0\end{array}$}} & \multirow{2}{*}{\multicolumn{2}{|c|}{$\begin{array}{l}\text { Ausprägung } \\
1\end{array}$}} & \multirow{2}{*}{\multicolumn{2}{|c|}{$\begin{array}{l}\text { Fehlende } \\
\text { Werte }\end{array}$}} \\
\hline \multirow[t]{2}{*}{ MZP1 } & \multirow[t]{2}{*}{ MZP2 } & \multirow[t]{2}{*}{ MZP3 } & & & 1 & & & & & & & & & & & & & & & \\
\hline & & & $\mathrm{N}$ & $\%$ & $\mathrm{~N}$ & $\%$ & $\mathrm{~N}$ & $\%$ & $\mathrm{~N}$ & $\%$ & $\mathrm{~N}$ & $\%$ & $\mathrm{~N}$ & $\%$ & $\mathrm{~N}$ & $\%$ & $\mathrm{~N}$ & $\%$ & $\mathrm{~N}$ & $\%$ \\
\hline a_vor_b1 & b_vor_b1 & c_vor_b1 & 343 & 77.08 & 95 & 21.35 & 7 & 1.57 & 49 & 84.48 & 7 & 12.07 & 2 & 3.45 & 24 & 72.73 & 8 & 24.24 & 1 & 3.03 \\
\hline a_vor_b2 & b_vor_b2 & c_vor_b2 & 358 & 80.45 & 80 & 17.98 & 7 & 1.57 & 51 & 87.93 & 5 & 8.62 & 2 & 3.45 & 28 & 84.85 & 4 & 12.12 & 1 & 3.03 \\
\hline a_vor_b3 & b_vor_b3 & c_vor_b3 & 357 & 80.22 & 81 & 18.20 & 7 & 1.57 & 49 & 84.48 & 7 & 12.07 & 2 & 3.45 & 27 & 81.82 & 5 & 15.15 & 1 & 3.03 \\
\hline a_vor_b4 & b_vor_b4 & c_vor_b4 & 249 & 55.96 & 189 & 42.47 & 7 & 1.57 & 38 & 65.51 & 18 & 31.04 & 2 & 3.45 & 22 & 66.67 & 10 & 30.30 & 1 & 3.03 \\
\hline a_vor_bs & b_vor_bs & c_vor_bs & 417 & 93.71 & 21 & 4.72 & 7 & 1.57 & 56 & 96.55 & -- & -- & 2 & 3.45 & 30 & 90.90 & 2 & 6.06 & 1 & 3.03 \\
\hline a_vor_b5 & b_vor_b5 & c_vor_b5 & 298 & 66.97 & 140 & 31.46 & 7 & 1.57 & 34 & 58.62 & 22 & 37.93 & 2 & 3.45 & 17 & 51.51 & 15 & 45.45 & 1 & 3.03 \\
\hline
\end{tabular}

\begin{tabular}{|c|c|c|c|c|c|c|}
\hline \multirow{4}{*}{$\begin{array}{l}\text { Itembezeichnung } \\
\text { Gesamt }\end{array}$} & \multicolumn{6}{|c|}{ Gesamt $(N=536)$} \\
\hline & \multirow{2}{*}{\multicolumn{2}{|c|}{$\begin{array}{l}\text { Ausprägung } \\
0\end{array}$}} & \multirow{2}{*}{\multicolumn{2}{|c|}{$\begin{array}{l}\text { Ausprägung } \\
1\end{array}$}} & \multicolumn{2}{|c|}{ Fehlende Werte } \\
\hline & & & & & & \\
\hline & $\mathrm{N}$ & $\%$ & $\mathrm{~N}$ & $\%$ & $\mathrm{~N}$ & $\%$ \\
\hline vor_b1 & 416 & 77.61 & 110 & 20.52 & 10 & 1.87 \\
\hline vor_b2 & 437 & 81.53 & 89 & 16.60 & 10 & 1.87 \\
\hline vor_b3 & 433 & 80.78 & 93 & 17.35 & 10 & 1.87 \\
\hline vor_b4 & 309 & 57.65 & 217 & 40.49 & 10 & 1.87 \\
\hline vor_bs & 503 & 93.84 & 23 & 4.29 & 10 & 1.87 \\
\hline vor_b5 & 349 & 65.11 & 177 & 33.02 & 10 & 1.87 \\
\hline
\end{tabular}




\subsubsection{Kontakt mit Menschen mit Behinderung: Quantität}

Kurzbezeichnung Variablenname

Instruktion

Skalierung (Antwortformat)

Instrument a_vor ko/b_vor ko/c_vor ko

a_vor_ko: Wie viel Kontakt hattest du vor deinem FSJ/BFD schon mit Menschen mit Behinderung oder psychischen Erkrankungen?

b_vor_ko: Wie viel Kontakt hattest du bis jetzt deinem FSJ/BFD schon mit Menschen mit Behinderung oder psychischen Erkrankungen?

c_vor_ko: Wie viel Kontakt hattest du insgesamt deinem FSJ/BFD schon mit Menschen mit Behinderung oder psychischen Erkrankungen?

Endpolbeschriftung; 1 = sehr wenig, $(\ldots), 5=$ sehr viel

a_vor_ko: Hauptfragebogen Messzeitpunkt 1, Zusatzbogen Erstteilnahme Messzeitpunkt 2 und 3

b_vor_ko: Hauptfragebogen Messzeitpunkt 2

c_vor_ko: Hauptfragebogen Messzeitpunkt 3

\begin{tabular}{|c|c|c|c|c|c|c|c|c|c|c|c|c|c|c|c|}
\hline \multirow[t]{3}{*}{ Itembezeichnung } & \multirow{2}{*}{\multicolumn{2}{|c|}{$\begin{array}{l}\text { Ausprägung } \\
1\end{array}$}} & \multirow{2}{*}{\multicolumn{2}{|c|}{$\begin{array}{l}\text { Ausprägung } \\
2\end{array}$}} & \multirow{2}{*}{\multicolumn{2}{|c|}{$\begin{array}{l}\text { Ausprägung } \\
3\end{array}$}} & \multirow{2}{*}{\multicolumn{2}{|c|}{$\begin{array}{l}\text { Ausprägung } \\
4\end{array}$}} & \multirow{2}{*}{\multicolumn{2}{|c|}{$\begin{array}{l}\text { Ausprägung } \\
5\end{array}$}} & \multirow{2}{*}{\multicolumn{2}{|c|}{$\begin{array}{l}\text { Fehlende } \\
\text { Werte }\end{array}$}} & \multirow{2}{*}{\multicolumn{3}{|c|}{ Gesamt }} \\
\hline & & & & & & & & & & & & & & & \\
\hline & $\mathrm{N}$ & $\%$ & $\mathrm{~N}$ & $\%$ & $\mathrm{~N}$ & $\%$ & $\mathrm{~N}$ & $\%$ & $\mathrm{~N}$ & $\%$ & $\mathrm{~N}$ & $\%$ & $\mathrm{~N}$ & MW & $\mathrm{SD}$ \\
\hline a_vor_ko & 174 & 39.10 & 99 & 22.25 & 83 & 18.65 & 65 & 14.61 & 50 & 11.24 & 6 & 1.35 & 445 & 2.50 & 1.37 \\
\hline b_vor_ko & 20 & 34.48 & 18 & 31.03 & 8 & 13.79 & 7 & 12.07 & 3 & 5.17 & 2 & 3.45 & 58 & 2.20 & 1.21 \\
\hline c_vor_ko & 12 & 36.36 & 8 & 24.24 & 5 & 15.15 & 5 & 15.15 & 2 & 6.06 & 1 & 3.03 & 33 & 2.28 & 1.30 \\
\hline
\end{tabular}




\subsubsection{Kontakt mit Menschen mit Behinderung: Valenz}

Kurzbezeichnung Variablenname

Instruktion

Skalierung (Antwortformat)

Instrument a_vor bw/b_vor_bw/c_vor_bw

a_vor_bw: Wie bewertest du die Erfahrungen, die du vor deinem FSJ/BFD mit Menschen mit Behinderung oder psychischen Erkrankungen gemacht hast?

b_vor_bw: Wie bewertest du die Erfahrungen, die du bis jetzt deinem FSJ/BFD mit Menschen mit Behinderung oder psychischen Erkrankungen gemacht hast?

c_vor_bw: Wie bewertest du die Erfahrungen, die du insgesamt in deinem FSJ/BFD mit Menschen mit Behinderung oder psychischen Erkrankungen gemacht hast?

Endpolbeschriftung; $1=$ sehr negativ, $(\ldots), 5=$ sehr positiv

a_vor_bw: Hauptfragebogen Messzeitpunkt 1, Zusatzbogen Erstteilnahme Messzeitpunkt 2 und 3 b_vor_bw: Hauptfragebogen Messzeitpunkt 2

c_vor_bw: Hauptfragebogen Messzeitpunkt 3

\begin{tabular}{|c|c|c|c|c|c|c|c|c|c|c|c|c|c|c|c|}
\hline \multirow[t]{3}{*}{ Itembezeichnung } & \multirow{2}{*}{\multicolumn{2}{|c|}{$\begin{array}{l}\text { Ausprägung } \\
1\end{array}$}} & \multirow{2}{*}{\multicolumn{2}{|c|}{$\begin{array}{l}\text { Ausprägung } \\
2\end{array}$}} & \multirow{2}{*}{\multicolumn{2}{|c|}{$\begin{array}{l}\text { Ausprägung } \\
3\end{array}$}} & \multirow{2}{*}{\multicolumn{2}{|c|}{$\begin{array}{l}\text { Ausprägung } \\
4\end{array}$}} & \multirow{2}{*}{\multicolumn{2}{|c|}{$\begin{array}{l}\text { Ausprägung } \\
5\end{array}$}} & \multirow{2}{*}{\multicolumn{2}{|c|}{$\begin{array}{l}\text { Fehlende } \\
\text { Werte }\end{array}$}} & \multirow{2}{*}{\multicolumn{3}{|c|}{ Gesamt }} \\
\hline & & & & & & & & & & & & & & & \\
\hline & $\mathrm{N}$ & $\%$ & $\mathrm{~N}$ & $\%$ & $\mathrm{~N}$ & $\%$ & $\mathrm{~N}$ & $\%$ & $\mathrm{~N}$ & $\%$ & $\mathrm{~N}$ & $\%$ & $\mathrm{~N}$ & MW & $\mathrm{SD}$ \\
\hline a_vor_bw & 3 & .56 & 23 & 4.29 & 200 & 37.31 & 174 & 32.46 & 104 & 19.40 & 32 & 5.97 & 536 & 3.70 & .87 \\
\hline b_vor_bw & 1 & .24 & 6 & 1.45 & 80 & 19.32 & 156 & 37.68 & 154 & 37.20 & 17 & 4.11 & 414 & 4.15 & .81 \\
\hline c_vor_bw & 2 & .58 & 3 & .87 & 54 & 15.70 & 113 & 32.85 & 158 & 45.93 & 14 & 4.07 & 344 & 4.28 & .81 \\
\hline
\end{tabular}




\subsubsection{Einstellungsveränderung}

Kurzbezeichnung Variablenname

Instruktion

Skalierung (Antwortformat)

Instrument b_aend/c_aend

Hat sich deine Einstellung zu Menschen mit Behinderung/psychischer Erkrankung seit Beginn deines FSJ verändert?

Endpolbeschriftung; 1 = überhaupt nicht verändert, $(\ldots), 5=$ sehr stark verändert

Hauptfragebogen Messzeitpunkt 2 und 3

\begin{tabular}{|c|c|c|c|c|c|c|c|c|c|c|c|c|c|c|c|c|}
\hline & \multirow[t]{2}{*}{ Itembezeichnung } & \multicolumn{2}{|c|}{$\begin{array}{l}\text { Ausprägung } \\
1\end{array}$} & \multicolumn{2}{|c|}{$\begin{array}{l}\text { Ausprägung } \\
2\end{array}$} & \multicolumn{2}{|c|}{$\begin{array}{l}\text { Ausprägung } \\
3\end{array}$} & \multicolumn{2}{|c|}{$\begin{array}{l}\text { Ausprägung } \\
4\end{array}$} & \multicolumn{2}{|c|}{$\begin{array}{l}\text { Ausprägung } \\
5\end{array}$} & \multicolumn{2}{|c|}{$\begin{array}{l}\text { Fehlende } \\
\text { Werte }\end{array}$} & \multicolumn{3}{|c|}{ Gesamt } \\
\hline & & $\mathrm{N}$ & $\%$ & $\mathrm{~N}$ & $\%$ & $\mathrm{~N}$ & $\%$ & $\mathrm{~N}$ & $\%$ & $\mathrm{~N}$ & $\%$ & $\mathrm{~N}$ & $\%$ & $\mathrm{~N}$ & MW & SD \\
\hline MZP2 & b_aend & 69 & 16.67 & 56 & 13.53 & 83 & 20.05 & 107 & 25.85 & 75 & 18.12 & 24 & 5.80 & 414 & 3.16 & 1.37 \\
\hline MZP3 & c_aend & 48 & 13.95 & 34 & 9.88 & 62 & 18.02 & 103 & 29.94 & 84 & 24.42 & 13 & 3.78 & 344 & 3.42 & 1.36 \\
\hline
\end{tabular}




\section{Literaturverzeichnis}

Aron, A. \& Fraley, B. (1999). Relationship closeness as including other in the self: Cognitive underpinnings and measures. Social Cognition, 17 (2), 140-160.

Ajzen, I., \& Fishbein, M. (2005). The influence of attitudes on behavior. In D. Albarracín, B. T. Johnson \& M. P. Zanna (Hrsg.), The handbook of attitudes (S. 173-221). Mahwah, NJ: Erlbaum.

Allport, G. W. (1954). The nature of prejudice. Cambridge: Addison-Wesley.

Antonak, R. F. (1981). Prediction of attitudes toward disabled persons. A multivariate analysis. The Journal of General Psychology, 104 (1), 119-123.

Antonak, R. F. \& Harth, R. (1994). Psychometric analysis and revision of the Mental Retardation Attitude Inventory. Mental Retardation, 32 (4), 272-280.

Autorengruppe Bildungsberichterstattung (2016). Bildung in Deutschland 2016. Ein indikatorengestützter Bericht mit einer Analyse zu Bildung und Migration. Bielefeld: wbv.

Autorengruppe Bildungsberichterstattung (2018). Bildung in Deutschland 2018. Ein indikatorengestützter Bericht mit einer Analyse zu Bildung und Migration. Bielefeld: wbv.

Aydin, N., Pfundmair, M., Agthe, M., Lermer, E. \& Frey, D. (2016). Stigma, Stigmatisierung und Ausgrenzung. In H.-W. Bierhoff, D. Frey, C. F. Graumann, N.-P. Birbaumer, J. Kuhl, W. Schneider et al. (Hrsg.), Ensyyklopädie der Sozialpsychologie, Soriale Motive und soziale Einstellungen (S. 675-704). Göttingen: Hogrefe Verlag für Psychologie.

Bandura, Albert (1971). Psychological modeling. Chicago: Aldine \& Atherton.

Baglieri, S. \& Shapiro, A. (2012). Disability studies and the inclusive classroom. New York, NY: Routledge.

Barr, J. J. \& Bracchitta, K. (2012). Attitudes toward individuals with disabilities: The effects of age, gender, and relationship. Journal of Relationships Research (3), 10-17.

Bem, D. J. (1972). Self-perception theory. In L. Berkowitz (Hrsg.), Advances in experimental social psychology (Bd. 6, S. 1-62). New York, NY: Academic Press.

Bogardus, E. S. (1933). A social distance scale. Sociology and Social Research (17), 265271.

Bolt, D. (Hrsg.) (2014). Changing social attitudes toward disability: Perspectives from historical, cultural, and educational studies (Routledge Advances in Disability Studies). London: Routledge.

Bouton, M. E. (2007). Learning and behavior: A contemporary synthesis. Sunderland, MA: Sinauer Associates.

Bosse, S. \& Spörer, N. (2014). Erfassung der Einstellung und der Selbstwirksamkeit von Lehramtsstudierenden zum inklusiven Unterricht. Empirische Sonderpädagogik (4), 279-299.

Bühl, A. (2018). SPSS. Einfübrung in die moderne Datenanalyse ab SPSS 25 (Pearson Studium - Scientific Tools, 16., akt. Aufl.). München: Pearson.

Coats, S., Smith, E. R., Claypool, H. M. \& Banner, M. J. (2000). Overlapping mental representations of self and in-group: Reaction time evidence and its relationship with explicit measures of group identification. Journal of Experimental Social Psychology 36 (3), 304-315. 
Cloerkes, G. (2007). Soziologie der Behinderten. Eine Einfübrung (3., neu bearb. u. erw. Aufl.). Heidelberg: Universitätsverlag Winter.

Degener, T. \& Diehl, E. (Hrsg.) (2015). Handbuch Bebindertenrechtskonvention. Teilhabe als Menschenrecht. Inklusion als gesellschaftliche Aufgabe (Schriftenreihe der Bundeszentrale für Politische Bildung, Bd. 1506). Bonn: BpB.

Engels, D., Leucht, M. \& Machalowski, G. (2008). Evaluation des freiwilligen sozialen Jahres und des freiwilligen ökologischen Jahres (Empirische Studien zum bürgerschaftlichen Engagement). Wiesbaden: Springer VS.

Eagly, A. H. \& Chaiken, S. (1998). Attitude structure and function. In D. T. Gilbert, S. T. Fiske, \& G. Lindzey (Hrsg.), The handbook of social psychology (S. 269-322). New York, NY: McGraw-Hill.

Eid, M. (1999). Lineare Strukturgleichungsmodelle. In B. Strauß, H. Haag \& M. Kolb (Hrsg.), Datenanalyse in der Sportwissenschaft (S. 427-454). Schondorf: Hofmann.

Fazio, R. H. (1990). Multiple processes by which attitudes guide behavior: The mode model as an integrative framework. In Zanna, M. (Hrsg.), Advances in experimental social psychology (S. 75-109). San Diego, CA: Academic Press.

Festinger, L. (1957). A theory of cognitive dissonance. Stanford, CA: Stanford University Press.

Findler, L., Vilchinsky, N. \& Werner, Shirli (2007). The Multidimensional Attitudes Scale Toward Persons With Disabilities (MAS). Construction and validation. Rehabilitation Counseling Bulletin, 50 (3), 166-176.

Fishbein, M. \& Ajzen, I. (2010). Predicting and changing behavior. New York, NY: Psychology Press.

Forlin, C., Earle, C., Loreman, T., \& Sharma, U (2001). The Sentiments, Attitudes, and Concerns about Inclusive Education Revised (SACIE-R) Scale for measuring pre-service teachers' perceptions about inclusion. Exceptionality Education International, 21 (3), 50-65.

Frechen, M., Meyer, K. \& Willems, A. S. (i. E.). Wie verändern Kontakt und Kontakt(un-)sicherheit Einstellungen gegenüber Menschen mit Behinderung? Befunde einer Studie zum Freiwilligen Sozialen Jahr (EFBI). In A. S. Willems (Hrsg.), „Warum und Wozu?" Motivation und Einstellung in (außer)schulischen Bildungsprozessen. Göttingen: Universitätsverlag (Erziehungswissenschaftliche Schriften).

Gebhardt, M., Schwab, S., Reicher, H., Ellmeier, B., Gmeiner, S., Rossmann, P. et al. (2011). Einstellungen von LehrerInnen zur schulischen Integration von Kindern mit einem sonderpädagogischen Förderbedarf in Österreich. Empirische Sonderpädagogik (4), 275-290.

Gething, L. \& Wheeler, B. (1992). The Interaction with Disabled Persons Scale. A new australian instrument to measure attitudes towards people with disabilities. Australian Journal of Psychology, 44 (2), 75-82.

Glaser, T. \& Bohner, G. (2016). Einstellungsänderung. In H.-W. Bierhoff \& D. Frey (Hrsg.), Enzyklopädie der Sozialpsychologie, Soziale Motive und soziale Einstellungen (S. 617-652). Göttingen: Hogrefe.

Hellmich, F., Görel, G. \& Schwab, S. (2016). Einstellungen und Motivation von Lehramtsstudentinnen und -studenten in Bezug auf den inklusiven Unterricht in 
der Grundschule. Ein Vergleich zwischen Deutschland und Österreich. Empirische Sonderpädagogik (1), 67-85.

Hodgetts, D. \& Stolte, O. (2016). Social distance. In T. Teo (Hrsg.), Encyclopedia of critical psychology (S. 1776-1778). New York, NY: Springer.

Hooper, D., Coughlan, J. \& Mullen, M. (2008). Structural Equation Modelling: Guidelines for Determining Model Fit. Electronic Journal of Business Research Methods, 6 (1), 53-60.

Hu, L.-t. \& Bentler, P. M. (1999). Cutoff criteria for fit indexes in covariance structure analysis: Conventional criteria versus new alternatives. Structural Equation Modeling: a Multidisciplinary Journal, 6 (1), 1-55.

Huth, S., Aram, E., Wagner, S., Engels, D. \& Maur, C. (2015). Abschlussbericht der gemeinsamen Evaluation des Gesetzes über den Bundesfreiwilligendienst (BFDG) und des Gesetzes zur Förderung von Jugendfreinilligendiensten (JFDG). Berlin: Bundesministerium für Familie, Senioren, Frauen und Jugend (Hrsg.).

Jerusalem, M. \& Hopf, D. (Hrsg.) (2002). Selbstwirksamkeit und Motivationsprozesse in Bildungsinstitutionen [Themenheft]. Zeitschrift für Pädagogik, 44. Weinheim: Beltz Juventa.

Kastl, J. M. (2017). Einführung in die Soziologie der Behinderung (2., völlig überarb. u. erw. Aufl.). Wiesbaden: Springer VS.

Kessler, T. \& Fritsche, I. (2018). Sozialpsychologie. Wiesbaden: Springer.

Kline, R. B. (2011). Principles and practice of structural equation modeling. New York, NY: Guilford Press.

Kopietz, R. \& Echterhoff, G. (2016). Einstellungen. In H.-W. Bierhoff \& D. Frey (Hrsg.), Ensyyklopädie der Sozialpsychologie, Soziale Motive und soziale Einstellungen (S. 581-616). Göttingen: Hogrefe.

Kreuz, A. (2002). Einstellungen gegenüber Menschen mit einer geistigen Behinderung. Analyse und Weiterentwicklung von Einstellungsinstrumenten. Dissertation, Universität Wien.

Kuckartz, U., Rädiker, S., Ebert, T. \& Schehl, J. (2013). Statistik. Eine verständliche Einführung (Lehrbuch, 2., überarb. Aufl.). Wiesbaden: Springer VS.

Kuhl, J., Moser, V., Schäfer, L. \& Redlich, H. (2013). Zur empirischen Erfassung von Beliefs von Förderschullehrerinnen und -lehrern. Empirische Sonderpädagogik (1), 324.

Kunz, A., Luder, R. \& Moretti, M. (2010). Die Messung von Einstellungen zur Integration (EZI). Empirische Sonderpädagogik (3), 83-94.

Lindemann, H. (2016). Konstruktion und empirische Validierung eines Instrumentes zur Erfassung der Einstellungen von Schülerinnen und Schülern gegenüber Peers mit Beeinträchtigung, anderer Herkunft und niedrigem sozioökonomischem Status. Empirische Sonderpädagogik (1), 5-21.

Lorenz, J., Stubbe, T. C., Krieg, M. \& Renftel, K. J. (2020). Einstellungen und Erwartungen von Lehramtsstudierenden zu schulischer Inklusion. Befunde einer quantitativen Befragung an den Standorten Göttingen, Braunschweig und Hannover. In K. Rabenstein, T. C. Stubbe \& K.-P. Horn (Hrsg.), Inklusion und Gymnasium. Studien zu Perspektiven von Lehrkräften und Studierenden (S. 77-105). Göttingen: Universitätsverlag (Erziehungswissenschaftliche Studien, Band 5). 
Maio, Gregory R. \& Haddock, Geoffrey (2015): The psychology of attitudes and attitude change (2. Aufl.). Los Angeles, CA: Sage.

Marsh, H. W., Hau, K.-T. \& Wen, Z. (2004). In Search of golden rules: Comment on hypothesis-testing approaches to setting cutoff values for fit indexes and dangers in overgeneralizing Hu and Bentler's (1999) findings. Structural Equation Modeling: a Multidisciplinary Journal, 11 (3), 320-341.

McElvany, N., Schwabe, F., Hartwig, S. J. \& Igler, J. (2018). Inklusive Lehr-LernSettings. Einstellungen und Motivation von Lehrkräften. Zeitschrift für Pädagogik, 64 (6), 831-851.

Meyer, K., Schröter, A. \& Bierschwale, C. (2021). Behinderung als Differenzkategorie in der quantitativen Einstellungsforschung - eine kritische Auseinandersetzung mit standardisierten Messinstrumenten. In S. Gabriel, P. Leinhos, K. Kotzyba, D. Matthes, K. Meyer \& M. Völcker (Hrsg.), Soziale Differenz und Reifizierung. Theoretische Zugänge und forschungspraktische Bearbeitungen (Studien zur Schul- und Bildungsforschung (SZSBF)) (S. 101-134). Wiesbaden: Springer VS.

Meyer, K. \& Willems, A. S. (i. E.). Verändern sich Einstellungen zu Behinderung durch Kontakterfahrungen und Bildungsseminare? Eine quantitative Längsschnittanalyse zu Freiwilligendiensten. In S. Fränkel, M. Grünke, T. Hennemann, D. Hövel, C. Melzer \& K. Ziemen, S. Fränkel, M. (Hrsg.), Teilhabe in allen Lebensbereichen? Ein Blick zurück und nach vorn. Bad Heilbrunn: Klinkhardt.

Meyer, K. \& Willems, A. S. (i. E.). Verändern sich Einstellungen zu Behinderung im Verlauf von Freiwilligendiensten? Eine Längsschnittanalyse latenter Profile. In A. S. Willems (Hrsg.), „Warum und Wozu? "Motivation und Einstellung in (außer)schulischen Bildungsprozessen. Göttingen: Universitätsverlag (Erziehungswissenschaftliche Schriften).

McGuire, W. J. (1969). The nature of attitudes and attitude change. In G. Lindzey \& E. Aronson (Hrsg.), The handbook of social psychology (S. 136-314). Reading, MA: Addison-Wesley.

McManus, J. L., Feyes, K. J. \& Saucier, D. A. (2010). Contact and knowledge as predictors of attitudes toward individuals with intellectual disabilities. Journal of Social and Personal Relationships, 28, 579-590.

Moosbrugger, H. (2012). Klassische Testtheorie (KTT). In H. Moosbrugger \& A. Kelava (Hrsg.), Testtheorie und Fragebogenkonstruktion (Springer-Lehrbuch, Bd. 15, S. 103-117). Berlin, Heidelberg: Springer Berlin Heidelberg.

Moosbrugger, H. \& Kelava, A. (Hrsg.). (2012). Testtheorie und Fragebogenkonstruktion (Springer-Lehrbuch). Berlin Heidelberg: Springer.

Moosbrugger, H. \& Schermelleh-Engel, K. (2012). Exploratorische (EFA) und Konfirmatorische Faktorenanalyse (CFA). In H. Moosbrugger \& A. Kelava (Hrsg.), Testtheorie und Fragebogenkonstruktion (Springer-Lehrbuch, S. 325-343). Berlin Heidelberg: Springer.

Muthén, L. K. \& Muthén, B. O. (2017). Mplus. Statistical analysis with latent variables. Los Angeles, CA: UCLA.

Nohl, A.-M. (2017). Interview und Dokumentarische Methode. Anleitungen für die Forschungspraxis. Wiesbaden: Springer VS. 
Paulus, C. (2013). Einstellungen zu Inklusion. Die deutsche Fassung des MTAI. Wiesbaden: Universität des Saarlandes.

Petty R. E. \& Cacioppo J. T. (1986). Communication and persuasion. Central and peripheral routes to attitude change. New York, NY: Springer.

Pettigrew, T. F. (1998). Intergroup contact theory. Annual Review of Psychology, 49 (1), 65-85.

Pit-ten Cate, I. M., Markova, M., Krischler, M. \& Krolak-Schwerdt, S. (2018). Promoting inclusive education: The role of teachers' competence and attitudes. Insights into Learning Disabilities, 15 (1), 49-63.

Powell, D. L. \& Powell, R. G. (2016). Classroom communication and diversity. Enhancing instructional practice. New York, NY: Routledge.

Rahrbach, A., Wüstendörfer, W. \& Arnold, T. (1998). Untersuchung zum Freiwilligen Sozialen Jahr (Schriftenreihe des Bundesministeriums für Familie, Senioren, Frauen und Jugend, Bd. 157, 1. Aufl.). Stuttgart: Kohlhammer.

Rauschenbach, T. \& Liebig, R. (2002): Freiwilligendienste - Wege in die Zukunft. Gutachten zur Lage und Zukeunft der Freiwilligendienste für den Arbeitskereis Bürgergesellschaft und Aktivierender Staat der Friedrich-Ebert-Stiftung. Bonn: Friedrich-Ebert-Stiftung (Hrsg.).

Ruberg, C. \& Porsch, R. (2017). Einstellungen von Lehramtsstudierenden und Lehrkräften zur schulischen Inklusion. Ein systematisches Review deutschsprachiger Forschungsarbeiten. Zeitschrift für Pädagogik, 63 (4), 393-456.

Schermelleh-Engel, K., Moosbrugger, H. \& Müller, H. (2003). Evaluating the fit of structural equation models: Tests of significance and descriptive goodness-of-fit measures. Methods of Psychological Research Online, 8 (2), 23-74.

Schmitz, G. S., Schwarzer, R. (1999). Proaktive Einstellung: Konstruktbeschreibung und psychometrische Analysen. Zeitschrift für Empirische Pädagogik, 13, 3-27.

Schreiber, J. B., Nora, A., Stage, F. K., Barlow, E. A. \& King, J. (2006). Reporting structural equation modeling and confirmatory factor analysis results: A review. The Journal of Educational Research, 99 (6), 323-338.

Schubert, T. W. \& Otten, S. (2002). Overlap of self, ingroup, and outgroup: pictorial measures of self-categorization. Self and Identity, 1 (4), 353-376.

Schwab, S. \& Seifert, S. (2015). Einstellungen von Lehramtsstudierenden und Pädagogikstudierenden zur schulischen Inklusion. Ergebnisse einer quantitativen Untersuchung. Zeitschrift für Bildungsforschung, 5 (1), 73-87.

Schwarzer, R. (Hrsg.) (1999). Skalen zur Erfassung von Lebrer- und Schülermerkmalen. Dokumentation der psychometrischen Verfabren im Rabmen der wissenschaftlichen Begleitung des Modellversuchs Selbstwirksame Schulen. Berlin: R. Schwarzer.

Seifert, K. H. \& Bergmann, C. (1983). Entwicklung eines Fragebogens zur Messung der Einstellungen. Heilpädagogische Forschung, 10 (3), 290-320.

Seifert, K. H. \& Stangl, W. (1981). Einstellungen zu Körperbebinderten und ibrer beruflichsozialen Integration. Bern: Huber.

Seifried, S. \& Heyl, V. (2016). Konstruktion und Validierung eines Einstellungsfragebogens zu Inklusion für Lehrkräfte (EFI-L). Empirische Sonderpädagogik (1), 22-35.

Sibley, C. G. \& Barlow, F. K. (Hrsg.) (2017). The cambridge handbook of the psychology of prejudice. Cambridge: Cambridge University Press. 
Sivo, S. A., Fan, X., Witta, E. L. \& Willse, J. T. (2006). The search for "optimal” cutoff properties: Fit index criteria in structural equation modeling. The Journal of Experimental Education, 74 (3), 267-288.

Statista GmbH (2018). Entwicklung der Anzabl der Freiwilligendienste in Deutschland von 2001 bis 2009. URL: https://de.statista.com/statistik/daten/studie/164502/umfrage/anzahl-derfreiwilligendienste-in-deutschland/ (letzter Zugriff 15.11.2019).

Stoiber, K. C., Gettinger, M. \& Goetz, D. (1998). Exploring factors influencing parents' and early childhood practitioners' beliefs about inclusion. Early Childhood Research Quarterly, 13 (1), 107-124.

Sturm, T. \& Wagner-Willi, M. (Hrsg.) (2018). Handbuch schulische Inklusion (utb Erziehungswissenschaft, Sonderpädagogik, Bd. 4959). Opladen: Verlag Barbara Budrich.

Völcker, M., Meyer, K. \& Jörke, D. (2019). Erkenntnistheoretische Grundlagen von Mixed Methods: Aktuelle Diskurslinien und forschungspraktische Perspektiven. In J. Lüdemann \& A. Otto (Hrsg.), Triangulation und Mixed Methods. Theoretische und forschungspraktische Reflexionen (S. 103-139). Wiesbaden: Springer VS.

Waldschmidt, A. (2005). Disability Studies: individuelles, soziales und/oder kulturelles Modell von Behinderung? Psychologie und Gesellschaftskritik, 29 (1), 9-31.

Wark, C. \& Galliher, J. F. (2007). Emory bogardus and the origins of the social distance scale. The American Sociologist, 38 (4), 383-395.

Watson, N., Roulstone, A. \& Thomas, C. (Hrsg.) (2014). Routledge handbook of disability studies. London: Routledge.

Werning, R. (2014). Stichwort: Schulische Inklusion. Zeitschrift für Erz̨iehungswissenschaft, $17(4), 601-623$.

Werth, L. \& Mayer, J. (2008). Sozialpsychologie. Berlin: Springer.

West, S. G., Taylor, A. B. \& Wu, W. (2012). Model fit and model selection in structural equation modeling. In R. H. Hoyle (Hrsg.), Handbook of Structural Equation Modeling (S. 209-231). New York, NY: Guilford Press.

Willems, A. S. \& Meyer, K. (2019). Welche Rolle spielen Einflussfaktoren vor und während des Studiums für die Überzeugungen von Lehramtsstudierenden zu schulischer Inklusion? In T. Ehmke, P. Kuhl \& M. Pietsch (Hrsg.). Lehrer. Bildung. Gestalten. Beiträge zur empirischen Forschung in der Lehrerbildung (S. 71-84). Weinheim: Beltz Juventa.

Woll, A. (2017). Kontaktbedingungen zwischen Menschen mit und obne Bebinderung als Prädiktoren von Einstellungen zu Inklusion. Dissertation, Pädagogische Hochschule Heidelberg.

Wood, W. (2000) Attitude change: persuasion and social influence. Annual Review of Psychology, 51, 539-570.

Yuker, H. E., Block, J. R., \& Younng, J. H. (1966). The measurement of attitudes toward disabled persons. Albertson, NY: Human Resources Center.

Zajonc, R. B. (1968). Attitudinal effects of mere exposure. Journal of Personality and Social Psychology, 9 (2), 1-27. 
ISSN: 2198-2384

doi:10.17875/gup2021-1761 